\title{
An Improved Cutting Plane Method for Convex Optimization, Convex-Concave Games and its Applications*
}

\author{
Haotian Jiang ${ }^{\dagger}$ Yin Tat Lee Zhao Song $^{\ddagger}$ Sam Chiu-wai Wong $₫$
}

\begin{abstract}
Given a separation oracle for a convex set $K \subset \mathbb{R}^{n}$ that is contained in a box of radius $R$, the goal is to either compute a point in $K$ or prove that $K$ does not contain a ball of radius $\epsilon$. We propose a new cutting plane algorithm that uses an optimal $O(n \log (\kappa))$ evaluations of the oracle and an additional $O\left(n^{2}\right)$ time per evaluation, where $\kappa=n R / \epsilon$.

- This improves upon Vaidya's $O\left(\mathrm{SO} \cdot n \log (\kappa)+n^{\omega+1} \log (\kappa)\right)$ time algorithm [Vaidya, FOCS 1989a] in terms of polynomial dependence on $n$, where $\omega<2.373$ is the exponent of matrix multiplication and $\mathrm{SO}$ is the time for oracle evaluation.

- This improves upon Lee-Sidford-Wong's $O\left(\mathrm{SO} \cdot n \log (\kappa)+n^{3} \log ^{O(1)}(\kappa)\right)$ time algorithm [Lee, Sidford and Wong, FOCS 2015] in terms of dependence on $\kappa$.

For many important applications in economics, $\kappa=\Omega(\exp (n))$ and this leads to a significant difference between $\log (\kappa)$ and poly $(\log (\kappa))$. We also provide evidence that the $n^{2}$ time per evaluation cannot be improved and thus our running time is optimal.

A bottleneck of previous cutting plane methods is to compute leverage scores, a measure of the relative importance of past constraints. Our result is achieved by a novel multi-layered data structure for leverage score maintenance, which is a sophisticated combination of diverse techniques such as random projection, batched low-rank update, inverse maintenance, polynomial interpolation, and fast rectangular matrix multiplication. Interestingly, our method requires a combination of different fast rectangular matrix multiplication algorithms.

Our algorithm not only works for the classical convex optimization setting, but also generalizes to convex-concave games. We apply our algorithm to improve the runtimes of many interesting problems, e.g., Linear Arrow-Debreu Markets, Fisher Markets, and Walrasian equilibrium.
\end{abstract}

\footnotetext{
*A preliminary version of this paper appears in Proceedings of the 52nd ACM Symposium on Theory of Computing (STOC 2020). The authors would like to express their sincere gratitude to matrix multiplicationer Josh Alman and Jeroen Zuiddam for their patient and answers of our exponential number of questions about fast matrix multiplication. ${ }^{\dagger}$ jhtdavid@uw.edu. University of Washington.

${ }^{\ddagger}$ yintat@uw. edu. University of Washington and Microsoft Research Redmond. Research supported in part by NSF Awards CCF-1740551, CCF-1749609, and DMS-1839116.

$\S$ zhaos@ias.edu. Princeton University and Institute for Advanced Study. Work done while visiting University of Washington.

"samwon@microsoft. com. Microsoft Research Redmond.
} 


\section{Contents}

1 Introduction $\quad 3$

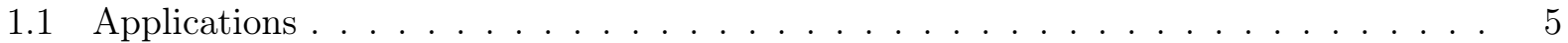

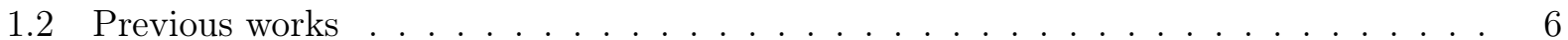

1.3 Lee-Sidford-Wong method $(\mathrm{LSW}) \ldots \ldots \ldots \ldots \ldots$

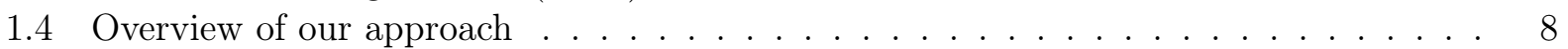

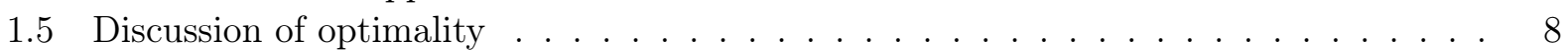

1.6 Related works . . . . . . . . . . . . . . . . . . . . . . 9

2 Our Techniques $\quad 10$

2.1 Efficient approximation of changes in leverage scores . . . . . . . . . . . . 10

2.2 Layered data structure . . . . . . . . . . . . . . . . . . . . . . . 10

2.3 Batched low-rank update . . . . . . . . . . . . . . . . . . 11

2.4 Illustration of our analysis . . . . . . . . . . . . . . . . . . . . . . . 12

2.5 Much faster rectangular matrix multiplication implies deterministic cutting plane

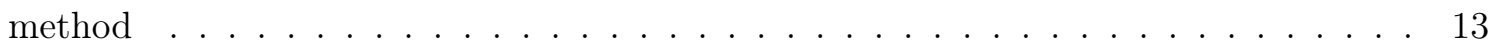

$\begin{array}{ll}\text { References } & 15\end{array}$

3 Preliminaries $\quad 22$

3.1 Notations . . . . . . . . . . . . . . . . . . . . . . . . . 22

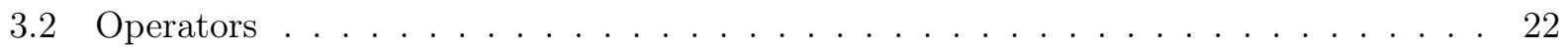

3.3 Different types of running time . . . . . . . . . . . . . . . . . . 22

3.4 Basic results on matrices . . . . . . . . . . . . . . . . . . . . . 23

3.5 Fast matrix multiplication . . . . . . . . . . . . . . . . . . . 23

3.6 Multiple variable polynomial interpolation . . . . . . . . . . . . . 23

4 Perturbed Volumetric Center Cutting Plane Method 26

$5 \quad$ Main Data Structure for Leverage Score Maintenance 27

6 Batched Low Rank Update $\quad 30$

6.1 Simple low rank update . . . . . . . . . . . . . . . . . . 30

6.2 Batched low rank update . . . . . . . . . . . . . . . . 34

7 Simple Deterministic Leverage Score Maintenance 39

7.1 Main result . . . . . . . . . . . . . . . . . . . . . . . . 39

7.2 Approximate leverage score's moving . . . . . . . . . . . . . . . . 40

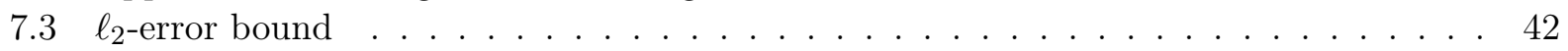

8 Complicated Randomized Leverage Score Maintenance 46

8.1 Main result . . . . . . . . . . . . . . . . . . . . 46

8.2 Leverage score's moving . . . . . . . . . . . . . . . . . . . . . . 46

8.3 Running time anlaysis . . . . . . . . . . . . . . . . . . . 50

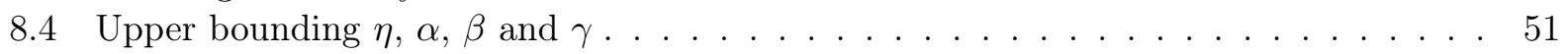

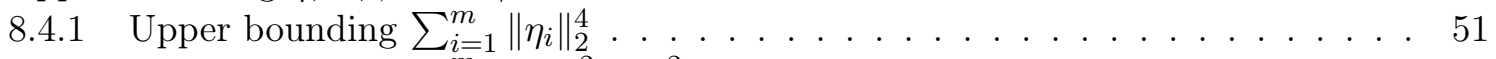

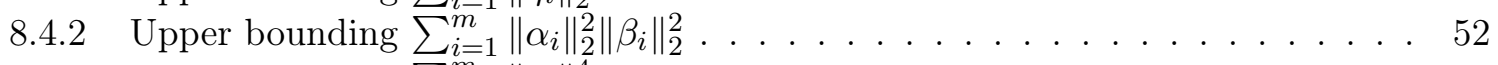

8.4.3 Upper bounding $\sum_{i=1}^{m}\left\|\gamma_{i}\right\|_{2}^{4} \ldots \ldots \ldots \ldots \ldots \ldots$ 
8.5 Variance upper bound for random Gaussian matrices . . . . . . . . . . . . . . 55

8.5.1 A variance bound for random Gaussian matrices . . . . . . . . . . . . . . 55

8.5.2 Variance bound for $\eta^{\top} \eta, \alpha^{\top} \beta$ and $\gamma^{\top} \gamma \ldots \ldots \ldots \ldots$

8.6 Error upper bound for discrete sampling . . . . . . . . . . . . 57

A Perturbed Volumetric Center Cutting Plane Method 59

A.1 Our faster implementation via leverage score maintenance . . . . . . . . . . . . 60

B Modified Projection Maintenance $\quad 64$

C Cutting Plane Method for Convex Minimization and Saddle Point Problems 66

C.1 Notations and definitions . . . . . . . . . . . . . . . . . . 66

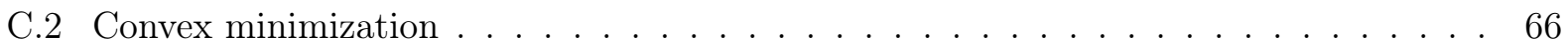

C.3 Convex-concave games . . . . . . . . . . . . . . . . . . . . . 66

C.4 Applying cutting plane method to convex-concave games . . . . . . . . . . . . 68

C.5 Cutting plane method for convex-concave games: generating solutions . . . . . . 68

$\begin{array}{ll}\text { D Applications of Cutting Plane Method } & \mathbf{7 4}\end{array}$

D.1 Linear Arrow-Debreu markets . . . . . . . . . . . . . . . . . . . . . . . . . 74

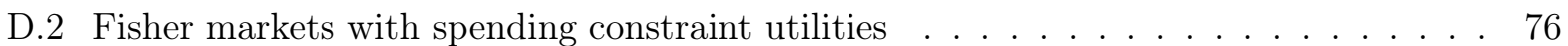

D.3 Walrasian equilibrium for general buyer valuations and fixed supply . . . . . . . 78 


\section{Introduction}

The cutting plane methods are a class of optimization primitives for solving Linear and Convex Programming, which is encapsulated by the feasibility problem of finding a point in a given convex set $K$ equipped with a separation oracle. In each iteration, cutting plane methods query the separation oracle which returns a hyperplane separating the query point from $K$. Since Khachiyan's breakthrough result [Kha80] on the ellipsoid method, cutting plane methods have played a central role in theoretical computer science, showing that a plethora of problems from diverse areas admit polynomial time algorithms. Early prominent examples include linear programming and submodular function minimization.

While more applications of the cutting plane methods are still being discovered to this date, progress on faster cutting plane methods had stagnated until the recent work of Lee, Sidford and Wong [LSW15]. Prior to their work, cutting plane methods were considered too slow to be of relevance in theory or in practice (other than establishing polynomiality). [LSW15] debunked this by showing how their cutting plane method is faster than tailor-made algorithms for a long list of well-studied problems.

The fastest algorithm before their work was Vaidya's algorithm [Vai89a], which runs in $O\left(n^{\omega+1}\right)$ time. Here $\omega<2.373$ is the exponent of matrix multiplication [CW87, Wil12, DS13, LG14]. Leveraging recent advances in optimization and numerical linear algebra, LSW lowered the dependence on the dimension $n$ at the expense of additional log factors in the accuracy $1 / \kappa$, namely $\log ^{2}(\kappa)$. The following table compares the runtimes of Vaidya's and LSW methods, which both achieve the optimal number of oracle calls of $n \log (\kappa)$.

\begin{tabular}{|l|l|l|}
\hline Authors & \#Oracle Calls & Runtimes \\
\hline \hline Vaidya & $n \log (\kappa)$ & $n^{\omega+1} \log (\kappa)$ \\
\hline Lee-Sidford-Wong & $n \log (\kappa)$ & $n^{3} \log O(1)(n) \log ^{3}(\kappa)$ \\
\hline
\end{tabular}

This extra overhead of $\log ^{2}(\kappa)$, as exemplified by various problems in combinatorial optimization in their paper, translates into only a log-squared factor in the maximum value $M$ of the input by taking $\epsilon=O(1 / M)$. This is because solutions to (polynomial-time solvable) problems in combinatorial optimization are guaranteed to be integral. Despite being a nuisance, this small overhead is relatively mild and can even be absorbed completely for unweighted problems and strongly polynomial time algorithms. Indeed, armed with this faster cutting plane method, they improved the state-of-the-art running times for a host of problems such as semidefinite programming, matroid intersection and submodular minimization.

Importance of $\log (\kappa)$ vs $\log ^{3}(\kappa)$. For many other problems including linear programming and market equilibrium computation, $\epsilon$ must be taken as $1 / M^{O(n)}$ resulting in an additional factor $n^{2}$ between $\log (\kappa)$ and $\log ^{3}(\kappa)$. For these applications, LSW is slower than Vaidy's by a factor of $\widetilde{O}\left(n^{4-\omega}\right)$. This raises a natural question: is there a cutting plane method that simultaneously runs in $O(n \log (\kappa))$ calls and $O\left(n^{3} \log O(1)(n) \log (\kappa)\right)$ time? That is, can we achieve the best of both worlds of Vaidya's and LSW methods in terms of the dependence on $n$ and $\kappa$ ?

In this paper, we answer this question in the affirmative. Somewhat surprisingly, we are able to remove $\log ^{O(1)}(n)$ dependence in LSW as well.

Theorem 1.1 (Main result). There is a cutting plane method which runs in time $O(n \cdot \mathrm{SO} \log (\kappa)+$ $\left.n^{3} \log (\kappa)\right)$, where SO is the time complexity of the separation oracle. 


\begin{tabular}{|l|l|l|l|}
\hline Reference & Year & Algorithm & Complexity \\
\hline \hline$[$ Sho77, YN76, Kha80] & 1979 & Ellipsoid Method & $n^{2} \mathrm{SO} \log (\kappa)+n^{4} \log (\kappa)$ \\
\hline$[$ KTE88, NN89] & 1988 & Inscribed Ellipsoid & $n \mathrm{SO} \log (\kappa)+(n \log (\kappa))^{4.5}$ \\
\hline$[$ Vai89a] & 1989 & Volumetric Center & $n \mathrm{SO} \log (\kappa)+n^{\omega+1} \log (\kappa)$ \\
\hline$[$ AV95] & 1995 & Analytic Center & $n \mathrm{SO} \log ^{2}(\kappa)+n^{\omega+1} \log ^{2}(\kappa)+(n \log (\kappa))^{2+\omega / 2}$ \\
\hline$[$ BV02] & 2004 & Random Walk & $n \mathrm{SO} \log (\kappa)+n^{7} \log (\kappa)$ \\
\hline$[$ LSW15] & 2015 & Hybrid Center & $n \mathrm{SO} \log (\kappa)+n^{3} \log ^{3}(\kappa)$ \\
\hline Theorem 1.1 & 2019 & Volumetric Center & $n \mathrm{SO} \log (\kappa)+n^{3} \log (\kappa)$ \\
\hline
\end{tabular}

Table 1: Algorithms for the Feasibility Problem. Let $\kappa=n R / \epsilon$. All methods can be used to solves a more general problem where only a membership oracle is given [LSV18].

\begin{tabular}{|l|l|l|l|}
\hline Reference & Year & \#Operations & Poly Type \\
\hline \hline [Eav75] & 1975 & Finite & Not poly \\
\hline$[$ Jai07] & 2007 & Polynomial & Weakly poly \\
\hline [Ye08] & 2008 & $n^{6} \log (n U)$ & Weakly poly \\
\hline [DPSV08] & 2008 & Polynomial & Weakly poly \\
\hline$[$ DM15] & 2015 & $n^{9} \log (n U)$ & Weakly poly \\
\hline$[$ DGM16] & 2016 & $n^{6} \log ^{2}(n U)$ & Weakly poly \\
\hline [GV19] & 2019 & $m n^{9} \log ^{2}(n)$ & Strongly poly \\
\hline Theorem 1.4 & 2019 & $m n^{2} \log (n U)$ & Weakly poly \\
\hline
\end{tabular}

Table 2: Linear Arrow-Debreu Markets. Let $n$ be the number of agents and $m$ the number of edges. Each operation of the given algorithms involves $O(n \log (n U))$-bit numbers.

\begin{tabular}{|l|l|l|l|}
\hline Reference & Year & \# Operations & Poly Type \\
\hline \hline [Vaz10] & 2010 & $n^{3}(n+m)^{2} \log (U) \cdot \mathcal{T}_{\text {max }}$ flow & Weakly poly \\
\hline [Vég16] & 2016 & $m n^{3}+m^{2}(m+n \log (n)) \log (m)$ & Strongly poly \\
\hline [Wan16] & 2016 & $m^{3} n+m^{2} \log (n)(n \log (n)+m)$ & Strongly poly \\
\hline Theorem 1.5 & 2019 & $m n^{2} \log (n U)$ & Weakly poly \\
\hline
\end{tabular}

Table 3: Fisher Markets with Spending Constraint Utilities. Let $n$ denote the total number of buyers and sellers, and $m$ the total number of segments. $\mathcal{T}_{\text {max }}$-flow denotes the number of operations needed for a max-flow computation. Each operation of the given algorithms involves $O(n \log (n U))$-bit numbers.

\begin{tabular}{|l|l|l|l|}
\hline Reference & Year & \# Operations & Poly Type \\
\hline \hline$[$ KJC82 $]$ & 1982 & Finite & Not poly \\
\hline$[$ Par99 $]$ & 1999 & Finite & Pseudo poly \\
\hline$[$ PU02] & 2002 & Finite & Pseudo poly \\
\hline$[$ AM02] & 2002 & Finite & Pseudo poly \\
\hline [dVSV07] & 2007 & Finite & Pseudo poly \\
\hline$[$ LW17] & 2017 & $n^{2} \mathcal{T}_{\mathrm{AD}} \log (S M n)+n^{6} \log O(1)(S M n)$ & Weak poly \\
\hline Theorem 1.6 & 2019 & $n^{2} \mathcal{T}_{\mathrm{AD}} \log (S M n)+n^{4} \log (S M n)$ & Weak poly \\
\hline
\end{tabular}

Table 4: Walrasian equilibrium for general buyer valuations and fixed supply. Let $\mathcal{T}_{\mathrm{AD}}$ denotes the runtime of aggregate demand oracle, $n$ denotes the number of goods. 
As with previous methods, our result achieves the asymptotic optimal oracle complexity of $O(n$. SO $\log (\kappa))$. Moreover, we conjecture that the runtime is likely the best possible. Note that in each iteration where a separation oracle call is made, even basic matrix operations require $O\left(n^{2}\right)$ time. Thus our runtime is essentially tight unless properties like sparsity can be surprisingly exploited to update the feasible region and compute the next query point for the separation oracle.

Our result is obtained by marrying Vaidya's method with recent advances in numerical linear algebra. Similar to LSW, we implement each iteration of Vaidya's via tools from fast numerical linear algebra. The main issue is that such tools rely on approximation and would lead to errors which must be carefully controlled.

Our first innovation is to run Vaidya's method in phases, each of which consist of a certain number of iterations. Between phases we "recompute" to eliminate the errors accumulated within a phase. Because of this recomputation we can afford to tolerate higher errors in an iteration. Secondly, we present a sophisticated data structure that enables us to implement each iteration of Vaidya's efficiently. Our data structure leverages recent advances on applying numerical techniques to optimization. We hope that these numerical tools, as well as our approach to applying them, would play a greater role in future development of optimization.

\subsection{Applications}

We highlight some of the key applications of our faster cutting plane method. Interestingly, even though our cutting plane method is a general purpose algorithm, we are able to improve the runtimes of tailor-made algorithms for various problems.

Using a standard reduction of convex minimization to the feasiblity problem ([Nem94] and Theorem 42 of [LSW15]), we can minimize a convex function with an optimal $\widetilde{O}(n)$ subgradient oracle calls and an additional $O\left(n^{2}\right)$ time per oracle call.

Theorem 1.2 (Informal version of Theorem C.1). Let $f$ be a convex function on $\mathbb{R}^{n}$ and $S$ be a convex set that contains a minimizer of $f$. Suppose we have a subgradient oracle for $f$ with cost $\mathcal{T}$ and $S \subset B(0, R)$. Using $B(0, R)$ as the initial polytope for our Cutting Plane Method, for any $0<\alpha<1$, we can compute $x \in S$ such that $f(x)-\min _{y \in S} f(y) \leq \alpha\left(\max _{y \in S} f(y)-\min _{y \in S} f(y)\right)$, with high probability in $n$ and with a running time of $O\left(\mathcal{T} \cdot n \log (\kappa)+n^{3} \log (\kappa)\right)$, where $\kappa=n \gamma / \alpha$ and $\gamma=R /(\operatorname{minwidth}(S))$.

Our convex minimization result can be further generalized to convex-concave games.

Theorem 1.3 (Informal version of Theorem C.9). Given convex sets $\mathcal{X} \subset B(0, R) \subset \mathbb{R}^{n}$ and $\mathcal{Y} \subset B(0, R) \subset \mathbb{R}^{m}$ such that both $\mathcal{X}$ and $\mathcal{Y}$ contain a ball of radius $r$. Let $f(x, y): \mathcal{X} \times \mathcal{Y} \rightarrow$ $\mathbb{R}$ be an L-Lipschitz function that is convex in $x$ and concave in $y$. Define $\kappa=\frac{n+m}{\epsilon} \frac{R}{r}$. For any $\epsilon \in(0,1 / 2]$, we can find $(\widehat{x}, \widehat{y})$ such that $\max _{y \in \mathcal{Y}} f(\widehat{x}, y)-\min _{x \in \mathcal{X}} f(x, \widehat{y}) \leq \epsilon \operatorname{Lr}$ in time $O\left(\mathcal{T} \cdot(n+m) \log (\kappa)+(n+m)^{3} \log (\kappa)\right)$ with high probability in $n+m$ where $\mathcal{T}$ is the cost of computing subgradient $\nabla f$.

Leveraging this improved dependence on $\kappa$ (and hence $\epsilon$ ), our cutting plane method can be used to improve the runtimes of a wide range of problems, especially those on market equilibrium computation (see Table 2, 3 and 4 for a summary of previous runtimes). In all our applications, $1 / \epsilon$ needs to be exponentially large in $n$ which renders the $\log (\kappa)$ factor polynomially large.

We show the following runtime improvement for the problem of computing a market equilibrium in linear exchange markets:

Theorem 1.4 (Informal version of Theorem D.5). There exists a weakly polynomial algorithm that computes a market equilibrium in linear exchange markets in time $O\left(m n^{2} \log (n U)\right)$. 
The celebrated result of Arrow and Debreu [AD54] shows the existence of a market equilibrium for a broad class of utility functions. Since then researchers have attempted to design efficient algorithms to compute market equilibria. One prominent special case of linear utilities has enjoyed significant attention as demonstrated by the long line of work in Table 2. Essentially, this problem can be captured by a convex program with linear constraints [DGV16] (see (19)). While this convex program exhibits certain advantageous features over previous ones [Cor89, Jai07, NP83], it had not led to improved runtimes since the objective is not separable [GV19]. Moreover, the number of variables in the convex program can be as large as $O\left(n^{2}\right)$, which prohibits a fast runtime for the cutting plane method if applied directly. Our approach in Theorem 1.4 is to transform the convex program into a convex-concave game with $O(n)$ variables and apply Theorem 1.3.

A similar technique can be applied to the problem of computing market equilibrium for Fisher markets with spending utility constraints where a convex program is given in [BDX10] (see (21)). However, directly transforming it into a convex-concave game does not reduce the dimension of the variables, as both the number of variables and constraints in the original convex program is $\Theta(m)$, where $m$ is the total number of segments and it can be much larger than $n^{2}$. In order to reduce the dimension of the convex-concave game to $O(n)$, we express part of the variables as functions of $O(n)$ variables and show that this does not increase the time of the first-order oracle. This leads to the following runtime improvement:

Theorem 1.5 (Informal version of Theorem D.7). There exists a weakly polynomial algorithm that computes a market equilibrium in Fisher markets with spending constraint utilities in time $O\left(m n^{2} \log (n U)\right)$.

Yet another economics application of our cutting plane method is the problem of computing a Walrasian equilibrium in a market with fixed supply. In this economy, buyers may have arbitrary valuation functions and we would like to compute prices so that the market clears, i.e. the aggregate demand of the buyers matches the fixed supply. Recently Paes Leme and Wong [LW17] gave a polynomial time algorithm for this problem provided that an equilbrium actually exists. They achieved this by showing that the cutting plane method can be modified so that the convex program for the equilibrium can be solved under the aggregate demand oracle.

By leveraging our faster cutting plane method we obtain an improved runtime:

Theorem 1.6 (Informal version of Theorem D.9). There is an algorithm that runs in time $O\left(n^{2} \mathcal{T}_{\mathrm{AD}} \log (S M n)+n^{4} \log (S M n)\right)$ for computing a market equilibrium in an economy with general buyer valuation in the aggregate demand model.

\subsection{Previous works}

The cutting plane methods solve the following feasbility problem that conveniently abstracts the applications to specific scenarios.

Feasibility Problem: Given a separation oracle for a set $K$ contained in a box of radius $R$ either find a point $x \in K$ or prove that $K$ does not contain a ball of radius $\epsilon$.

All cutting plane methods maintain a candidate region $\Omega$ and solve the feasibility problem by iteratively refining $\Omega$ based on the present $\Omega$ and the new separating hyperplane. In each iteration:

1. The separation oracle is queried at some point $x \in \Omega$.

2. If $x \in K$ we have solved the feasibility problem.

3. Otherwise, the separation oracle returns a separating hyperplane from which $\Omega$ is further refined 
and the next query point $x$ is computed.

Previous works differ in how $x$ is selected and how $\Omega$ is refined. For instance, the classic ellipsoid method maintains $\Omega$ as an ellipsoid and $x$ as its center. Given $\Omega$ and the new separating hyperplane, the new $\Omega$ is chosen to be the smallest ellipsoid containing their intersection. Table 1 lists the running times for solving the feasibility problem in the literature.

We focus our discussion on the trade-off between the oracle complexity and the runtime per iteration. The oracle complexity has a lower bound $\Omega(n \log (\kappa))$ [NY83]. While the ellipsoid method achieves a suboptimal $O\left(n^{2} \log (\kappa)\right)$ in oracle complexity, the runtime per iteration is $O\left(n^{2}\right)$ which is faster than all subsequent methods. The good runtime follows from the simple calculations needed to update the ellipsoid, whereas the suboptimal oracle complexity can be attributed to the "looseness" of maintaining only an ellipsoid as a proxy to the intersection of past separating half-spaces.

Indeed, one can attain the optimal oracle complexity $O\left(n^{2} \log (\kappa)\right)$ by maintaining all previous separating half-spaces. This is the random walk method [BV02] where the query point $x$ is chosen to be its (approximate) center of gravity. Updating $x$ involves performing a random walk in this polytope and is computationally expensive.

Other cutting methods improve oracle complexity by maintaining more fine-grained information about past separating hyperplanes in a way that is computationlly friendly. Of particular relevance is Vaidya's volumetric center method [Vai89a]. Vaidy's $\Omega$, similar to the random walk method, is also a polytope $\Omega=\left\{x \in \mathbb{R}^{n}: A x \geq b\right\}$. As the name suggests, the query point is chosen to be the volumetric center, which is the minimizer of the following convex function defined by the feasible region $\Omega$ :

$$
\frac{1}{2} \log \operatorname{det}\left(A^{\top} S_{x}^{-2} A\right) \text { where } S_{x}:=\operatorname{diag}(A x-b) \text { is the diagonal matrix of the slacks }
$$

Nevertheless, by judiciously including only a representative subset of previous half-spaces $a x \geq b$, Vaidya showed that the volumetric center and $\Omega$ can be updated by basic matrix operations which run in $O\left(n^{\omega}\right)$ time.

We defer the discussion of LSW to the next subsection, which explains how Vaidya's method can be sped up using machineries from numerical linear algebra.

\subsection{Lee-Sidford-Wong method (LSW)}

LSW's key observation is that Vaidya's method relies heavily on leverage scores, which measure the relative importance of each separating hyperplane. In Vaidya's method, naively updating leverage scores requires $O\left(n^{\omega}\right)$ time and is a bottleneck. Inspired by the work of Spielman and Srivistava [SS11], LSW attempted to address this by using random Johnson-Lindenstrauss [JL84] (JL) projection to approximate changes in leverage scores. Leverage scores can then be updated by summing over the differences.

Approximating leverage scores changes via JL projection however still requires solving a linear system which would still take $O\left(n^{\omega}\right)$ time. LSW further overcame this barrier by resorting to a

recent work that efficiently solves "slowly-changing" linear system in amortized $\widetilde{O}\left(n^{2}\right)$ time. Thus after paying $O\left(n^{\omega}\right)$ initially, they can solve such linear systems in $\widetilde{O}\left(n^{2}\right)$ time per iteration.

Error accumulation. Nevertheless, as an approximate method JL introduces errors which would accumulate across iterations. While Vaidya's method tolerates small errors in leverage scores, the total errors incurred in JL projection (as accumulated across iterations) would eventually become too big and destroy the performance guarantee of Vaidya's method. 
LSW handled this by modifying Vaidya's framework to take into account of the error in the convex function to be minimized. This approach gives rise to a "hybrid" center algorithm, which involves a complicated interplay of optimization and linear algebra. In particular, to reduce the error accumulated the error parameter $\epsilon_{0}$ in JL projection has to be as small as $\epsilon_{0}=1 / \log (\kappa)$. As the runtime of JL depends on $1 / \epsilon_{0}^{2}$, this unfortunately introduces the $\log ^{2} \kappa$ overhead in LSW runtime when compared to Vaidya's volumetric center method.

\subsection{Overview of our approach}

To achieve the desired $O\left(n \mathrm{SO} \log (\kappa)+n^{3} \log (\kappa)\right)$ runtime, we build a data structure that approximates changes in leverage scores to within $c$ in $\ell_{2}$ norm for small enough constant $c$. This would suffice for Vaidya's cutting plane method. The desired runtime then directly follows from the performance guarantee of the data structure, which handles each iteration in amortized $O\left(n^{2}\right)$ time. Designing such a data structure requires several new ideas to control the error accumulation issue.

Our data structure employs a layered approach where different layers are associated with different error tolerances, and achieve different accuracy-efficiency tradeoffs in approximating the changes in leverage scores. The more inner a layer is, the more error it can tolerate and the faster is the runtime. Whenever the error accumulated in a layer becomes too much, the layer above would take over and produce a finer error estimate which would, of course, be more time costly. But because of our layered approach, the higher layer is called on less often and afford to spend more time.

Such a layered approach further leads to the following issue. In the middle and outer layers, we batch the updates of multiple steps into one which allows us to make use of fast rectangular matrix multiplication. However, our algorithm needs to handle possibly exponential weight changes. We show there are not too many such weights and can be handled separately in groups of poly $\log (n)$ size using low-rank update formula.

Our data structure also draws on various numerical tools such as fast rectangular matrix multiplication, "tall" JL projection, preconditioning, inverse maintenance, and polynomial interpolation for approximating integrals.

A more in-depth discussion of our techniques can be found in Section 2.

\subsection{Discussion of optimality}

Similar to previous methods our cutting plane method achieves the optimal oracle complexity $n \log (\kappa)$ [NY83]. We present some evidence that our running time of $O\left(n^{3} \log (\kappa)\right)$ is also tight.

A bottleneck of Vaidya's method is to solve the inverse maintenance problem. Formally, given a sequence of positive vectors $w^{1}, w^{2}, \cdots w^{T}$, let $P(w)$ be defined as

$$
P(w)=\sqrt{W} A\left(A^{\top} W A\right)^{-1} A^{\top} \sqrt{W},
$$

where $W$ is the diagonal matrix such that $W_{i, i}=w_{i}$. The goal is to output a sequence of vectors $v^{1}, v^{2}, \cdots, v^{T}$ such that

$$
v^{t} \approx w^{t} \quad \text { and } P\left(v^{t}\right) \approx P\left(w^{t}\right), \forall t \in[T] .
$$

There is a long line of research on inverse maintenance and dynamic matrix data-structure problems [Kha80, Vai89b, San04, LS15, HKNS15, CLS19, LSZ19, Son19, BNS19, Bra20]. This task can be done naively by spending $n^{\omega}$ time so the goal is to achieve $o\left(n^{\omega}\right)$ amortized cost per iteration. For example, in the LP setting the number of iterations is $O(\sqrt{n})$ and Vaidya [Vai89b] combined fast matrix multiplication with inverse maintenance to achieve $O\left(n^{2}\right)$ amortized cost per iteration, 
which gives an $O\left(n^{2.5}\right)$ time algorithm. This remained a barrier until recent works [CLS19, LSZ19] combined sampling and sketching techniques with fast rectangular matrix multiplication and inverse matrix maintenance to give an $O\left(n^{\omega}\right)$ time algorithm.

One of the major computation required in each step is matrix-vector multiplication, e.g., $P(w) \cdot h$. Naively, this step takes $O\left(n^{2}\right)$ time per iteration. To achieve $o\left(n^{2}\right)$ amortized cost per iteration, previous works [CLS19, LSZ19] used an idea called "iterating and sketching" which was formally described in [Son19]. This idea is very different from the classical "sketch and solve" [CW13] and "guess a sketch" [RSW16]. The classical idea usually applying sketching matrices only once without modifying the solver itself. However, the "iterating and sketching" idea has to modify the solver and applying sketching/sampling matrices over each iteration.

In [CLS19], they use the diagonal sampling matrix $D \in \mathbb{R}^{n \times n}$ which has roughly $\sqrt{n}$ nonzeros. They use that sampling matrix to sample on the right hand side:

$$
\sqrt{W} A\left(A^{\top} W A\right)^{-1} A^{\top} \sqrt{W} \underbrace{D}_{\text {sample right }} h .
$$

In [LSZ19], they use the subsampled randomized Hadamard/Fourier matrix [LDFU13, PSW17] $R \in \mathbb{R}^{\sqrt{n} \times n}$. They use the sketching matrix to sketch on the left hand side:

$$
\underbrace{R^{\top} R}_{\text {sketch left }} \sqrt{W} A\left(A^{\top} W A\right)^{-1} A^{\top} \sqrt{W} h .
$$

Compared to the cutting plane method, LP is an easier maintenance task as the matrix $A$ is fixed throughout. In the cutting plane method, however, rows get inserted into or deleted from $A$ from continuously. One critical idea used in all previous works on LP [Vai89b, CLS19, LSZ19] is to delay low-rank updates on $\left(A^{\top} W A\right)^{-1}$. However, in the cutting plane method, the low rank updates to $A$ cannot be delayed. Thus it appears that previous techniques are inapplicable. Moreover, $n^{2}$ lower bounds have recently been established for natural matrix maintenance tasks (e.g. determinant, inverse) with row/column insertions/deletions under the Online Matrix-Vector conjecture (e.g. [HKNS15, BNS19]). Therefore, we believe our algorithm is tight and conjecture the following:

Conjecture 1.7. Solving the feasibility problem requires $\Omega\left(n^{3} \log (\kappa)\right)$ time. Hence our cutting plane method achieves the optimal runtime.

\subsection{Related works}

Leverage scores. Leverage scores are a fundamental concept in graph problems and numerical linear algebra. There are many works about how to approximate leverage scores [SS11, DMIMW12, CW13, NN13] or more general version of leverages, e.g. Lewis weights [Lew78, BLM89, CP15] and ridge leverage scores [CMM17]. From graph perspective, it was applied to solve max-flow [Mad13, Mad16], generate random spanning trees [Sch18], and sparsify graphs [SS11]. From matrix perspective, it was used to give matrix CUR decomposition [BW14, SWZ17, SWZ19] and tensor CURT decomposition [SWZ19]. From optimization perspective, it was used for approximating the John Ellipsoid [CCLY19], accelerating the kernel methods $\left[\mathrm{AKM}^{+} 17, \mathrm{AKM}^{+} 19\right]$, showing the convergence of the deep neural network [LSS ${ }^{+}$20], cutting plane methods, e.g. [Vai89a, LSW15] and this paper.

Linear Program. Linear Program is a fundamental problem in convex optimization and can be treated as an special case where one can apply the cutting plane method. There is a super long list of work focused on fast algorithms for linear program [Dan47, Kha80, Kar84, Vai87, Vai89b, LS14, LS15, Sid15, Lee16, CLS19, LSZ19, Son19, Bra20, BLSS20]. 
Membership oracle. Besides the separation oracle considered in this paper, there is another line of work on using the membership oracle to solve the feasibility problem [Pro96, KV06, LV06, GLS12, LSV18]. For a query point $x$, this oracle outputs $x \in K$ or $x \notin K$.

\section{Our Techniques}

Section 1.4 provided a quick overview. Here we take a deeper dive into our techniques.

\subsection{Efficient approximation of changes in leverage scores}

The key to fast maintenance of leverage scores is an efficient way to approximate their changes between consecutive steps. While a fine-grained approximation leads to an accurate approximation, the time to compute such an approximation might be unaffordable. On the other hand, a coarsegrained approximation can be efficiently computed, but might lead to accumulating errors that blow up after a small number of steps. This leads to a tradeoff between accuracy and efficiency.

Central to our data structure are a coarse-grained formula (Lemma 7.4) and a fine-grained formula for the change in leverage scores (Lemma 8.4). While the coarse-grained formula approximates the leverage score's change via a single integral, the fine-grained formula is a cocktail involving integrals, matrix inverse and matrix multiplication. For the coarse-grained formula, we simply estimate the integral by a single point along the integral (Lemma 7.5). For the fine-grained formula, however, the approximation (Lemma 8.10) is more involved and requires appropreiate numerical tools.

Our course-grained and fine-grained formulas lead to two different data structures for leverage score maintenance: a simple deterministic data structure that achieves low running time but introduces a large error in each step, and a more complicated randomized data structure that incurs small error each step at the cost of efficiency.

Observe that we are allowed to recompute the leverage scores exactly after every $O\left(n^{\omega-2+o(1)}\right)$ steps as exact computation of leverage scores takes $O\left(n^{\omega+o(1)}\right)$ time. Therefore it suffices to control the error accumulation in $O\left(n^{\omega-2+o(1)}\right)$ steps. To achieve this, both the simple and complicated data structures are crucial. While the simple data structure achieves the desired $O\left(n^{2}\right)$ time per step, the error accrued in the $O\left(n^{\omega-2+o(1)}\right)$ steps are too large for the cutting plane method. For the complicated data structure, we are able to achieve $\ell_{2}$-error $o(1)$ in $O\left(n^{\omega-2+o(1)}\right)$ steps, but the amortized running time would be $O\left(n^{2+o(1)}\right)$ per step.

\subsection{Layered data structure}

To achieve the best of both worlds of the simple and complicated data structures, we propose a data structure that combines both data structures and interpolates between accuracy and efficiency. Specifically, our data structure approximates changes in leverage scores to $\ell_{2}$-error within $1 / \log ^{O(1)}(n)$ which would suffice for Vaidya's cutting plane method and handles each iteration in amortized $O\left(n^{2}\right)$ time.

Our data structure employs a layered approach (see Figure 1) where different layers are associated with different error tolerances. The more inner a layer is, the more error it can tolerate and the faster is the runtime. Whenever the error accumulated in a layer becomes too high, the layer above would take over and produce a finer error estimate which would, of course, be more time costly. But because of our layered approach, the higher layer is called on less often and can afford to spend more time. More specifically, our layered data structure contains three layers. The inner and middle layers both employ the simple data structure to achieve computational efficiency while the outer layer uses the complicated data structure to ensure a low error. 
Our layered approach builds on several fundamental results on matrix multiplication which we summarize in Theorem 2.1. We remark that Vaidya [Vai89b] used the first result, a recent LP solver [CLS19] used the first two, while our cutting plane method crucially depends on all the results in the table.

Theorem 2.1 (Fast rectangular matrix multiplication results). For any $n, r>0$, denote $\mathcal{T}_{\text {mat }}(n, n, r)$ the time to compute the multiplication of an $n \times n$ matrix and an $n \times r$ matrix ${ }^{1}$. Then we have:

\begin{tabular}{|l|l|l|l|}
\hline Reference & $r$ & $\mathcal{T}_{\text {mat }}(n, n, r)$ & Layer \\
\hline \hline$[$ LG14 $]$ & $n$ & $O\left(n^{\omega+o(1)}\right)$ & Restart \\
\hline$[$ GU18 $]$ & $n^{0.31}$ & $O\left(n^{2+o(1)}\right)$ & Outer layer \\
\hline$[$ Cop82] & $n^{0.17}$ & $O\left(n^{2} \log ^{2} n\right)$ & Middle layer \\
\hline$[$ BD76] & $\log ^{O(1)} n$ & $O\left(n^{2}\right)$ & Inner layer \\
\hline
\end{tabular}

Our data structure also draws on various numerical tools such as fast rectangular matrix multiplication, "tall" JL projection, preconditioning, inverse maintenance, and polynomial interpolation for approximating integrals, which we discuss in more details below.

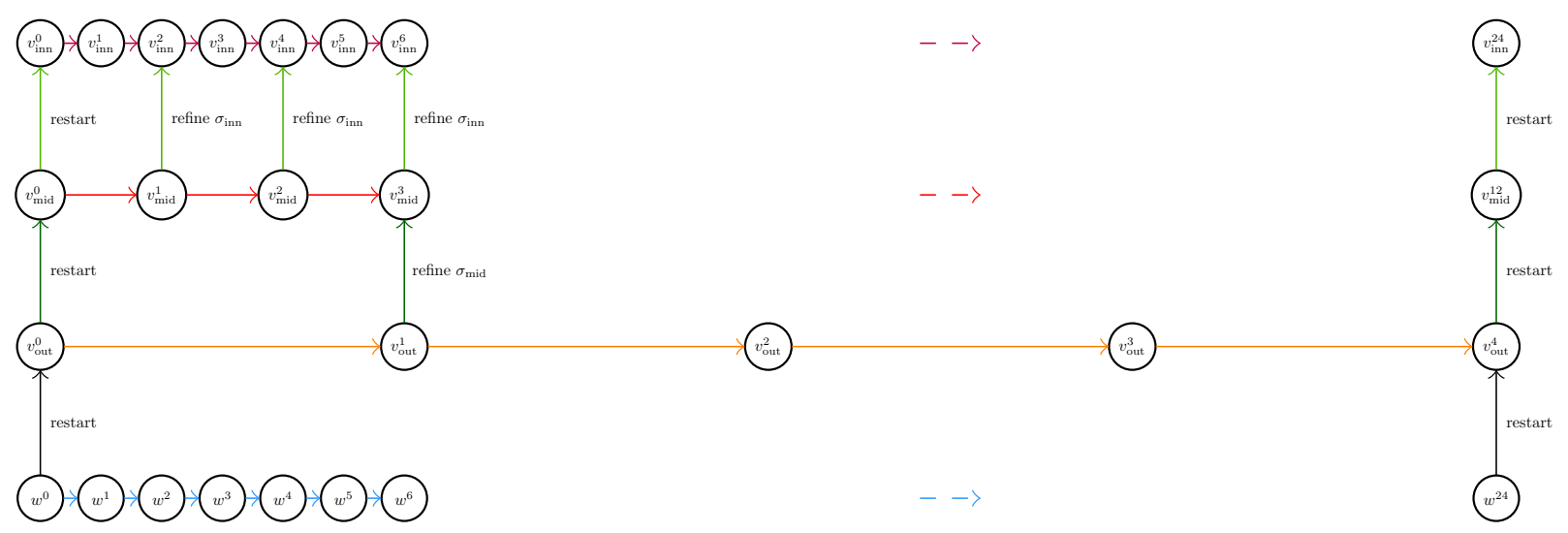

Figure 1: Illustration of our three-level data structure with $T_{\mathrm{inn}}=2, T_{\mathrm{mid}}=3$ and $T_{\text {out }}=4$ approximating the leverage scores of the sequence $\left\{w^{0}, w^{1}, \cdots\right\}$. The three levels maintain three approximate sequences $\left\{v_{\text {inn }}^{0}, v_{\text {inn }}^{1}, \cdots\right\},\left\{v_{\text {mid }}^{0}, v_{\text {mid }}^{1}, \cdots\right\}$ and $\left\{v_{\text {out }}^{0}, v_{\text {out }}^{1}, \cdots\right\}$, with errors $\epsilon_{\text {inn }}=\left\|\log (w)-\log \left(v_{\text {inn }}\right)\right\|_{\infty}$, $\epsilon_{\text {mid }}=\left\|\log (w)-\log \left(v_{\text {mid }}\right)\right\|_{\infty}$ and $\epsilon_{\text {out }}=\left\|\log (w)-\log \left(v_{\text {out }}\right)\right\|_{\infty}$ that satisfy $1 \gg \epsilon_{\text {inn }} \gg \epsilon_{\text {mid }} \gg \epsilon_{\text {out }}>0$. The inner level takes a step for every $w$-update, the middle step takes a step in every $T_{\text {inn }}$ inner steps, and the outer step takes a step in every $T_{\text {mid }}$ middle steps. The entire data structure is restarted after $T_{\text {out }}$ outer steps. Each middle step refines the inner approximation of leverage scores, and each outer step refines the approximation of both the inner and middle approximations of the leverage scores. For the actual choice of the parameters $T_{\mathrm{inn}}, T_{\mathrm{mid}}, T_{\mathrm{out}}$ and $\epsilon_{\mathrm{inn}}, \epsilon_{\mathrm{mid}}, \epsilon_{\mathrm{out}}$ in our data structure, see Table 5 .

\subsection{Batched low-rank update}

The layered approach in the previous subsection leads to the following technical hurdle. While the inner layer reacts to each update, the middle and outer layers perform one step only after a certain number of updates. In the cutting plane method, each update of the vector $w \in \mathbb{R}_{+}^{m}$ satisfies an $\ell_{2^{-}}$ closeness property: $\left\|\log \left(w^{\text {new }}\right)-\log (w)\right\|_{2}=O(1)$. For a sequence of $T$ updates $w^{(0)}, w^{(1)}, \cdots, w^{(T)}$,

\footnotetext{
${ }^{1}$ Note that $\mathcal{T}_{\text {mat }}(n, n, r)=\mathcal{T}_{\text {mat }}(n, r, n)$
} 
the $\ell_{2}$-closeness property is no longer satisfied for $w^{(0)}$ and $w^{(T)}$, and the weight changes between $w^{(0)}$ and $w^{(T)}$ can be exponential in $T$.

To resolve this issue, we employ a batched low-rank update method that computes a vector $v^{\text {mid }}$ that is $\ell_{\infty}$-close to the vector $w^{(T)}$ and an accurate estimate of the leverage score change $\sigma\left(v^{\text {mid }}\right)-\sigma\left(w^{0}\right)$. This accurate estimate is done by a low-rank update rule for matrices (Woodbury matrix identity). Unfortunately, the Woodbury formula involves the inverse of certain matrices but the inverse maintenance data structure only maintains the inverse of a nearby matrix. Therefore, we need to use the maintained inverse as a preconditioner to approximately compute the Woodbury formula. Due to the exponential changes in the weight, we need to solve certain linear systems to exponential accuracy and it can be too expensive even with a very good preconditioner (Lemma $6.2)$.

To rescue this preconditioner idea, we transform and split the sequence into pieces of size poly $\log (n)$. We ensure the weight changes by only a quasi-polynomial factor and this decreases the cost of solving linear systems to poly $\log (n)$ steps. Since we batch the task of handling poly $\log (n)$ weight changes into one rectangular matrix multiplication which can be performed in $O\left(n^{2}\right)$ time, we make sure the cost per weight change is exactly $O\left(n^{2}\right)$ time (Theorem 6.3).

\subsection{Illustration of our analysis}

We describe the numerical tools used in our analysis, and provide simple illustrations of our applications of these tools. The actual way in which they are used in Sections 7-8 are more involved.

Discrete sampling for multiple variable integrals The most standard way to approximate an integral is by discretization, which takes a weighted sum of the integrand over a set of points in the domain. Unlike common discretization tools like the trapezoidal method, for our purpose we need to interpolate multiple variable integral using a polynomial for higher accuracy (Theorem 3.10). We give a simple example to illustrate our application of polynomial interpolation for multiple variable integrals as follows. In our fine-grained formula of the leverage score change from $w$ to $w^{\text {new }}$, one of the integral terms is

$$
\sigma_{i, \mathrm{cts}}=\int_{0}^{1} \int_{0}^{1} \int_{0}^{1} \gamma_{i, s, t}^{\top} \gamma_{i, s^{\prime}, t} \mathrm{~d} s \mathrm{~d} s^{\prime} \mathrm{d} t
$$

where

$$
\gamma_{i, s, t}=\sqrt{W^{\text {mid }}-W^{\text {new }}} \cdot Q\left(y_{s, t}\right) \cdot\left(Z_{t}-X_{t}\right) \cdot Q\left(y_{s, t}\right) \cdot \sqrt{W^{\text {new }}} \cdot e_{i} .
$$

In order to approximate such an integral, we take a set $\mathcal{T} \subseteq[0,1]$ of $N=\log ^{O(1)}(n)$ points along the integration together with weights $\left\{\omega_{t}\right\}_{t \in \mathcal{T}}$ as in Theorem 3.10, and approximate the integral by

$$
\sigma_{i, \mathrm{dis}}=\sum_{t \in \mathcal{T}} \sum_{s \in \mathcal{S}} \sum_{s^{\prime} \in \mathcal{S}} \omega_{t} \omega_{s} \omega_{s^{\prime}} \gamma_{i, s, t}^{\top} \gamma_{i, s^{\prime}, t} .
$$

Theorem 3.10 then shows that the $\ell_{2}$-error can be bounded as $\left\|\sigma_{\text {cts }}-\sigma_{\text {dis }}\right\|_{2} \leq \operatorname{poly}(n) / 2^{2 N}$, which is negligible by our choice of $N=\log ^{O(1)}(n)$.

Projection maintenance and preconditioning Inverse maintenance was first proposed in [Kha80] as a method for solving "slowly-changing" linear system.

Given a sequence of positive vectors $w^{1}, w^{2}, \cdots w^{T} \in \mathbb{R}_{+}^{m}$, let $P(w)$ be defined as $P(w)=$ $\sqrt{W} A\left(A^{\top} W A\right)^{-1} A^{\top} \sqrt{W}$, where $W \in \mathbb{R}^{m \times m}$ is the diagonal matrix with $W_{i, i}=w_{i}$. The goal is 
to output a sequence of vectors $v^{1}, v^{2}, \cdots, v^{T}$ such that $(1-\epsilon) v^{t} \leq w^{t} \leq(1+\epsilon) v^{t}, \forall t \in[T]$, and efficiently computes $P\left(v^{t}\right) u$, for query vector $u \in \mathbb{R}^{n}$. The recent work of [CLS19] gave an efficient way to perform such a task. For our purpose, however, rather than just computing $P\left(v^{t}\right) u$, we also need explicit approximations to the matrices $Q(w)=A\left(A^{\top} W A\right)^{-1} A^{\top}$ and $M(w)^{-1}=\left(A^{\top} W A\right)^{-1}$. The matrices $Q(w)$ and $M(w)^{-1}$ appear frequently in our formula for the changes in leverage scores, and we need their approximations as pre-conditioners for accelerating the computation of certain matrix rectangular multiplication involving $Q(w)$ and $M(w)^{-1}$ (Lemma 6.1).

"Tall" JL \& fast rectangular matrix multiplication From the previous paragraph on discrete sampling, it suffices to compute $\gamma_{i, s, t}$ for all $i$, where

$$
\gamma_{i, s, t}=\sqrt{W^{\text {mid }}-W^{\text {new }}} \cdot Q\left(y_{s, t}\right) \cdot\left(Z_{t}-X_{t}\right) \cdot Q\left(y_{s, t}\right) \cdot \sqrt{W^{\text {new }}} \cdot e_{i} .
$$

Notice that computing $\gamma_{i, s, t}$ for all $i$ is essentially computing the matrix products

$$
\sqrt{W^{\text {mid }}-W^{\text {new }}} \cdot Q\left(y_{s, t}\right) \cdot\left(Z_{t}-X_{t}\right) \cdot Q\left(y_{s, t}\right) \cdot \sqrt{W^{\text {new }}}
$$

which would take $O\left(n^{\omega+o(1)}\right)$ time if computed exactly. To improve the time while ensuring keeping the error small, we invoke JL with dimension $n^{c}$ for small constant $c$ by computing

$$
\sigma_{i, \mathrm{jl}}=\sum_{t \in \mathcal{T}} \sum_{s \in \mathcal{S}} \sum_{s^{\prime} \in \mathcal{S}} \omega_{t} \omega_{s} \omega_{s^{\prime}} \gamma_{i, s, t}^{\top} R_{\gamma, s, s^{\prime}, t}^{\top} R_{\gamma, s, s^{\prime}, t} \gamma_{i, s^{\prime}, t}
$$

where $R_{\gamma, s, s^{\prime}, t} \in \mathbb{R}^{n^{c} \times n}$ is a random matrix. It is essential that $c$ is picked such that the rectangular matrix multiplication can be done in time roughly $n^{2}$. To obtain a small error, $\sigma_{i, j 1}$ should be a good estimate with a small variance. We note that $\sigma_{i, \mathrm{jl}}$ is indeed an unbiased estimator of $\sigma_{i, \text { dis }}$ and its variance can be bounded as $\sum_{i=1}^{m} \operatorname{Var}\left[\sigma_{i, j 1}\right] \leq O\left(\epsilon^{2} / n^{c}\right)$, where $\epsilon$ is the error for the projection maintenance used by the data structure (Sections 8.4 and 8.5). Leveraging fast rectangular matrix multiplication, we pick $c=0.31$ and $\epsilon=n^{-0.1}$. The variance would then be bounded by $n^{-0.51}$ which is sufficiently small after $n^{\omega-2+o(1)}$ steps before the data structure restarts.

\subsection{Much faster rectangular matrix multiplication implies deterministic cutting plane method}

Our algorithm crucially relies on different kinds of fast rectangular matrix multiplication results. We also show that if these results are improved, then we are able to get a deterministic cutting plane method immediately.

Corollary 2.2 (Informal version of Corollary 7.3). If $\mathcal{T}_{\text {mat }}(n, n, r)=O\left(n^{2} \log ^{O(1)}(n)\right)$ for $r=n^{\beta}$ with $\beta>2 / 3$, then there is a deterministic cutting plane method which runs in time

$$
O\left(n \cdot \mathrm{SO} \log (\kappa)+n^{3} \log (\kappa)\right),
$$

where $\mathrm{SO}$ is the time complexity of the separation oracle.

Let $\alpha$ denote the dual exponent of matrix multiplication, which is the largest number $\alpha>0$ such that $\mathcal{T}_{\text {mat }}\left(n, n, n^{\alpha}\right)=n^{2+o(1)}$. Let $\beta$ denote the largest number such that $\mathcal{T}_{\text {mat }}\left(n, n, n^{\beta}\right)=$ $n^{2} \log { }^{O(1)} n$. A very recent result by Christandl, Le Gall, Lysikov and Zuiddam [CGLZ20] showed the limitations of several tensor techniques: they proved that $\alpha<0.625$ for certain tensors. We believe our work initiated two interesting open questions in the area of fast matrix multiplication: 
(1) whether one can prove a better upper bound on $\beta$ (compared to $\alpha$ ) for certain tensor techniques, and (2) if there is a non-trivial inequality between $\beta$ and $\alpha$.

Organization. We introduce basic notations, backgrounds and tools in Section 3. In Section 4, we present the statement that Vaidya's cutting plane method tolerates perturbed leverage scores. We present our main data-structure for maintaining leverage scores in Section 5. Our main datastructure uses two different leverage score maintenance data-structures in Sections 7 and 8 with three different settings of the error parameter. Both our data-structures in Section 7 and 8 reply on the batched low rank algorithm in Section 6.

In Section A, we prove that Vaidya's cutting plane method tolerates perturbed leverage scores. We provide several modified versions of projection maintenance data-structures in Section B. Finally, we explain how to handle convex-concave game optimization in Section C, and present our applications to market equilibrium computations in Section D.

\section{Acknowledgments}

The authors would like to express their sincere gratitude to matrix multiplicationer Josh Alman for his patient and answers of our exponential number of questions about fast matrix multiplication.

The authors would like to thank Swati Padmanabhan for very useful discussions at the early stage of this project. The authors would like to thank Lijie Chen, Nikhil Devanur, Irit Dinur, Simon S. Du, Wei Hu, Jason Lee, Jerry Li, Ruoqi Shen, Aaron Schild, Aaron Sidford, Santosh Vempala, Xin Yang, Peilin Zhong, and Danyang Zhuo.

The authors would like to thank Josh Alman, Irit Dinur, Avi Wigderson, and Jeroen Zuiddam for useful discussion about limitation of fast matrix multiplication.

The authors would like to thank Sanjeev Arora, Alexandr Andoni, Ainesh Bakshi, Yangsibo Huang, Rajesh Jayaram, Ravindran Kanna, Michael Kapralov, Adam Klivans, Kai Li, Christos Papadimitriou, Eric Price, Daniel Roy, Clifford Stein, Omri Weinstein, David P. Woodruff, and Hengjie Zhang for asking interesting questions at the end of the talk of this paper.

This project was supported in part by NSF awards CCF-1749609, CCF-1740551, DMS-1839116, and Microsoft Research Faculty Fellowship.

This project was supported in part by Special Year on Optimization, Statistics, and Theoretical Machine Learning (being led by Sanjeev Arora) at Institute for Advanced Study. 


\section{References}

[AD54] Kenneth J Arrow and Gerard Debreu. Existence of an equilibrium for a competitive economy. Econometrica: Journal of the Econometric Society, pages 265-290, 1954.

[AKM+17] Haim Avron, Michael Kapralov, Cameron Musco, Christopher Musco, Ameya Velingker, and Amir Zandieh. Random Fourier features for kernel ridge regression: Approximation bounds and statistical guarantees. In International Conference on Machine Learning (ICML). https://arxiv.org/pdf/1804.09893.pdf, 2017.

[AKM+19] Haim Avron, Michael Kapralov, Cameron Musco, Christopher Musco, Ameya Velingker, and Amir Zandieh. A universal sampling method for reconstructing signals with simple Fourier transforms. In 51st Annual ACM SIGACT Symposium on Theory of Computing (STOC). https://arxiv.org/pdf/1812.08723.pdf, 2019.

[AM02] Lawrence M Ausubel and Paul R Milgrom. Ascending auctions with package bidding. Advances in Theoretical Economics, 1(1), 2002.

[AV95] David S Atkinson and Pravin M Vaidya. A cutting plane algorithm for convex programming that uses analytic centers. Mathematical Programming, 69(1-3):1-43, 1995.

[BD76] Roger W Brockett and David Dobkin. On the number of multiplications required for matrix multiplication. SIAM Journal on Computing, 5(4):624-628, 1976.

[BDX10] Benjamin Birnbaum, N Devanur, and Lin Xiao. New convex programs and distributed algorithms for fisher markets with linear and spending constraint utilities. Unpublished manuscript, 2010.

[BLM89] Jean Bourgain, Joram Lindenstrauss, and V Milman. Approximation of zonoids by zonotopes. Acta mathematica, 162(1):73-141, 1989.

[BLSS20] Jan van den Brand, Yin Tat Lee, Aaron Sidford, and Zhao Song. Solving tall dense linear programs in nearly linear time. In 52nd Annual ACM SIGACT Symposium on Theory of Computing (STOC). https://arxiv.org/pdf/2002.02304.pdf, 2020.

[BNS19] Jan van den Brand, Danupon Nanongkai, and Thatchaphol Saranurak. Dynamic matrix inverse: Improved algorithms and matching conditional lower bounds. In 60th Annual IEEE Symposium on Foundations of Computer Science (FOCS). https: //arxiv.org/pdf/1905.05067.pdf, 2019.

[Bra20] Jan van den Brand. A deterministic linear program solver in current matrix multiplication time. In ACM-SIAM Symposium on Discrete Algorithms (SODA). https: //arxiv.org/pdf/1910.11957.pdf, 2020.

[BV02] Dimitris Bertsimas and Santosh Vempala. Solving convex programs by random walks. In Proceedings of the thiry-fourth annual ACM symposium on Theory of computing (STOC), pages 109-115. ACM, 2002.

[BW14] Christos Boutsidis and David P Woodruff. Optimal cur matrix decompositions. In Proceedings of the 46th Annual ACM Symposium on Theory of Computing (STOC), pages 353-362. ACM, https://arxiv.org/pdf/1405.7910, 2014. 
[CCLY19] Michael B Cohen, Ben Cousins, Yin Tat Lee, and Xin Yang. A near-optimal algorithm for approximating the John ellipsoid. In Annual Conference on Learning Theory (COLT). https://arxiv.org/pdf/1905.11580.pdf, 2019.

[CGLZ20] Matthias Christandl, François Le Gall, Vladimir Lysikov, and Jeroen Zuiddam. Barriers for rectangular matrix multiplication. In arXiv preprint. https://arxiv.org/ 2003.03019.pdf, 2020.

[CLS19] Michael B Cohen, Yin Tat Lee, and Zhao Song. Solving linear programs in the current matrix multiplication time. In Proceedings of the 51st Annual ACM Symposium on Theory of Computing (STOC). https://arxiv.org/pdf/1810.07896.pdf, 2019.

[CMM17] Michael B Cohen, Cameron Musco, and Christopher Musco. Input sparsity time lowrank approximation via ridge leverage score sampling. In Proceedings of the TwentyEighth Annual ACM-SIAM Symposium on Discrete Algorithms (SODA), pages 17581777. SIAM, https://arxiv.org/pdf/1511.07263.pdf, 2017.

[Cop82] Don Coppersmith. Rapid multiplication of rectangular matrices. SIAM Journal on Computing, 11(3):467-471, 1982.

[Cor89] Bernard Cornet. Linear exchange economies. Cahier Eco-Math, Université de Paris, $1,1989$.

[CP15] Michael B. Cohen and Richard Peng. $\ell_{p}$ row sampling by lewis weights. In Proceedings of the Forty-Seventh Annual ACM on Symposium on Theory of Computing (STOC), pages 183-192. https://arxiv.org/pdf/1412.0588, 2015.

[CW87] Don Coppersmith and Shmuel Winograd. Matrix multiplication via arithmetic progressions. In Proceedings of the nineteenth annual ACM symposium on Theory of computing (STOC), pages 1-6. ACM, 1987.

[CW13] Kenneth L. Clarkson and David P. Woodruff. Low rank approximation and regression in input sparsity time. In Symposium on Theory of Computing Conference (STOC), pages 81-90. https://arxiv.org/pdf/1207.6365, 2013.

[Dan47] George B Dantzig. Maximization of a linear function of variables subject to linear inequalities. Activity analysis of production and allocation, 13:339-347, 1947.

[DGM16] Ran Duan, Jugal Garg, and Kurt Mehlhorn. An improved combinatorial polynomial algorithm for the linear arrow-debreu market. In Proceedings of the twenty-seventh annual ACM-SIAM symposium on Discrete algorithms (SODA), pages 90-106. SIAM, https://arxiv.org/pdf/1510.02694.pdf, 2016.

[DGV16] Nikhil R Devanur, Jugal Garg, and László A Végh. A rational convex program for linear arrow-debreu markets. volume 5(1), page 6. https://arxiv.org/pdf/1307. 8037 .pdf, 2016.

[DM15] Ran Duan and Kurt Mehlhorn. A combinatorial polynomial algorithm for the linear arrow-debreu market. Information and Computation, 243:112-132, 2015.

[DMIMW12] Petros Drineas, Malik Magdon-Ismail, Michael W Mahoney, and David P Woodruff. Fast approximation of matrix coherence and statistical leverage. Journal of Machine Learning Research, 13(Dec):3475-3506, 2012. 
[DPSV08] Nikhil R Devanur, Christos H Papadimitriou, Amin Saberi, and Vijay V Vazirani. Market equilibrium via a primal-dual algorithm for a convex program. Journal of the $A C M(J A C M), 55(5): 22,2008$.

[DS13] Alexander Munro Davie and Andrew James Stothers. Improved bound for complexity of matrix multiplication. Proceedings of the Royal Society of Edinburgh Section A: Mathematics, 143(2):351-369, 2013.

[dVSV07] Sven de Vries, James Schummer, and Rakesh V Vohra. On ascending vickrey auctions for heterogeneous objects. Journal of Economic Theory, 132(1):95-118, 2007.

[Eav75] B Curtis Eaves. A finite algorithm for the linear exchange model. Technical report, STANFORD UNIV CALIF SYSTEMS OPTIMIZATION LAB, 1975.

[GLS12] Martin Grötschel, László Lovász, and Alexander Schrijver. Geometric algorithms and combinatorial optimization, volume 2. Springer Science \& Business Media, 2012.

[GU18] Francois Le Gall and Florent Urrutia. Improved rectangular matrix multiplication using powers of the coppersmith-winograd tensor. In Proceedings of the Twenty-Ninth Annual ACM-SIAM Symposium on Discrete Algorithms (SODA), pages 1029-1046. SIAM, https://arxiv.org/pdf/1708.05622.pdf, 2018.

[GV19] Jugal Garg and László A Végh. A strongly polynomial algorithm for linear exchange markets. In Proceedings of the 51st Annual ACM SIGACT Symposium on Theory of Computing (STOC), pages 54-65. ACM, https://arxiv.org/pdf/1809.06266.pdf, 2019 .

[HKNS15] Monika Henzinger, Sebastian Krinninger, Danupon Nanongkai, and Thatchaphol Saranurak. Unifying and strengthening hardness for dynamic problems via the online matrix-vector multiplication conjecture. In Proceedings of the forty-seventh annual ACM symposium on Theory of computing (STOC), pages 21-30. ACM, https: //arxiv.org/pdf/1511.06773.pdf, 2015.

[IK94] Eugene Isaacson and Herbert Bishop Keller. Analysis of numerical methods. 1994.

[Jai07] Kamal Jain. A polynomial time algorithm for computing an arrow-debreu market equilibrium for linear utilities. SIAM Journal on Computing, 37(1):303-318, 2007.

[JL84] William B Johnson and Joram Lindenstrauss. Extensions of lipschitz mappings into a hilbert space. Contemporary mathematics, 26(189-206):1, 1984.

[Kar84] Narendra Karmarkar. A new polynomial-time algorithm for linear programming. In Proceedings of the sixteenth annual ACM symposium on Theory of computing (STOC), pages 302-311. ACM, 1984.

[Kha80] Leonid G Khachiyan. Polynomial algorithms in linear programming. USSR Computational Mathematics and Mathematical Physics, 20(1):53-72, 1980.

[KJC82] Alexander S Kelso Jr and Vincent P Crawford. Job matching, coalition formation, and gross substitutes. Econometrica: Journal of the Econometric Society, pages 14831504, 1982. 
[KTE88] Leonid G Khachiyan, Sergei Pavlovich Tarasov, and I. I. Erlikh. The method of inscribed ellipsoids. In Soviet Math. Dokl, volume 37, pages 226-230, 1988.

[KV06] Adam Tauman Kalai and Santosh Vempala. Simulated annealing for convex optimization. Mathematics of Operations Research, 31(2):253-266, 2006.

[LDFU13] Yichao Lu, Paramveer Dhillon, Dean P Foster, and Lyle Ungar. Faster ridge regression via the subsampled randomized hadamard transform. In Advances in neural information processing systems (NIPS), pages 369-377, 2013.

[Lee16] Yin Tat Lee. Faster algorithms for convex and combinatorial optimization. PhD thesis, Massachusetts Institute of Technology, 2016.

[Lew78] D. Lewis. Finite dimensional subspaces of $\ell_{p}$. Studia Mathematica, 63(2):207-212, 1978.

[LG14] François Le Gall. Powers of tensors and fast matrix multiplication. In Proceedings of the 39th international symposium on symbolic and algebraic computation (ISSAC), pages 296-303. ACM, 2014.

[LS14] Yin Tat Lee and Aaron Sidford. Path finding methods for linear programming: Solving linear programs in $O(\sqrt{\text { rank }})$ iterations and faster algorithms for maximum flow. In 55th Annual IEEE Symposium on Foundations of Computer Science (FOCS), pages 424-433. https://arxiv.org/pdf/1312.6677.pdf, https://arxiv.org/pdf/1312. 6713.pdf, 2014.

[LS15] Yin Tat Lee and Aaron Sidford. Efficient inverse maintenance and faster algorithms for linear programming. In 56th Annual IEEE Symposium on Foundations of Computer Science (FOCS), pages 230-249. https://arxiv.org/pdf/1503.01752.pdf, 2015.

[LSS $\left.{ }^{+} 20\right] \quad J a s o n$ Lee, Ruoqi Shen, Zhao Song, Mengdi Wang, and Zheng Yu. Leverage score sampling, neural tangent kernel, and kernel ridge regression. In Manuscript, 2020.

[LSV18] Yin Tat Lee, Aaron Sidford, and Santosh S Vempala. Efficient convex optimization with membership oracles. In Annual Conference on Learning Theory (COLT). https: //arxiv.org/pdf/1706.07357.pdf, 2018.

[LSW15] Yin Tat Lee, Aaron Sidford, and Sam Chiu-wai Wong. A faster cutting plane method and its implications for combinatorial and convex optimization. In 56th Annual IEEE Symposium on Foundations of Computer Science (FOCS), pages 1049-1065. https: //arxiv.org/pdf/1508.04874.pdf, 2015.

[LSZ19] Yin Tat Lee, Zhao Song, and Qiuyi Zhang. Solving empirical risk minimization in the current matrix multiplication time. In Annual Conference on Learning Theory (COLT). https://arxiv.org/pdf/1905.04447, 2019.

[LV06] László Lovász and Santosh Vempala. Fast algorithms for logconcave functions: Sampling, rounding, integration and optimization. In 47th Annual IEEE Symposium on Foundations of Computer Science (FOCS), pages 57-68. IEEE, 2006.

[LW17] Renato Paes Leme and Sam Chiu-wai Wong. Computing walrasian equilibria: fast algorithms and structural properties. In Proceedings of the Twenty-Eighth Annual 
ACM-SIAM Symposium on Discrete Algorithms (SODA), pages 632-651. SIAM, https://arxiv.org/pdf/1511.04032.pdf, 2017.

[Mad13] Aleksander Madry. Navigating central path with electrical flows: From flows to matchings, and back. In 54th Annual IEEE Symposium on Foundations of Computer Science (FOCS), pages 253-262. https://arxiv.org/pdf/1307.2205.pdf, 2013.

[Mad16] Aleksander Madry. Computing maximum flow with augmenting electrical flows. In 2016 IEEE 57th Annual Symposium on Foundations of Computer Science (FOCS), pages 593-602. IEEE, https://arxiv.org/pdf/1608.06016.pdf, 2016.

[Nem94] Arkadi Nemirovski. Efficient methods in convex programming. Lecture notes, 1994.

[Nem95] Arkadi Nemirovski. Information-based complexity of convex programming. Lecture Notes, 1995.

[NN89] YE Nesterov and AS Nemirovskii. Self-concordant functions and polynomial time methods in convex programming. preprint, central economic \& mathematical institute, ussr acad. Sci. Moscow, USSR, 1989.

[NN13] Jelani Nelson and Huy L Nguyên. OSNAP: Faster numerical linear algebra algorithms via sparser subspace embeddings. In 54th Annual IEEE Symposium on Foundations of Computer Science (FOCS), pages 117-126. IEEE, https://arxiv.org/pdf/1211. 1002, 2013.

[NP83] EI Nenakov and ME Primak. One algorithm for finding solutions of the arrow-debreu model. Kibernetica, 3:127-128, 1983.

[NY83] Arkadii Semenovich Nemirovsky and David Borisovich Yudin. Problem complexity and method efficiency in optimization. 1983.

[Par99] David C Parkes. ibundle: An efficient ascending price bundle auction. 1999.

[Pro96] V Yu Protasov. Algorithms for approximate calculation of the minimum of a convex function from its values. Mathematical Notes, 59(1):69-74, 1996.

[PSW17] Eric Price, Zhao Song, and David P. Woodruff. Fast regression with an $\ell_{\infty}$ guarantee. In International Colloquium on Automata, Languages, and Programming (ICALP), 2017.

[PU02] David C Parkes and Lyle H Ungar. An ascending-price generalized vickrey auction. 2002 .

[RSW16] Ilya Razenshteyn, Zhao Song, and David P Woodruff. Weighted low rank approximations with provable guarantees. In Proceedings of the 48th Annual Symposium on the Theory of Computing (STOC), 2016.

[San04] Piotr Sankowski. Dynamic transitive closure via dynamic matrix inverse. In 45th Annual IEEE Symposium on Foundations of Computer Science (FOCS), pages 509517. IEEE, 2004. 
[Sch18] Aaron Schild. An almost-linear time algorithm for uniform random spanning tree generation. In Proceedings of the 50th Annual ACM SIGACT Symposium on Theory of Computing (STOC), pages 214-227. ACM, https://arxiv.org/pdf/1711.06455. pdf, 2018.

[Sho77] Naum Z Shor. Cut-off method with space extension in convex programming problems. Cybernetics and systems analysis, 13(1):94-96, 1977.

[Sid15] Aaron Daniel Sidford. Iterative methods, combinatorial optimization, and linear programming beyond the universal barrier. PhD thesis, Massachusetts Institute of Technology, 2015.

[Son19] Zhao Song. Matrix Theory: Optimization, Concentration and Algorithms. PhD thesis, The University of Texas at Austin, 2019.

[SS11] Daniel A Spielman and Nikhil Srivastava. Graph sparsification by effective resistances. In SIAM Journal on Computing, volume 40(6), pages 1913-1926. https://arxiv. org/pdf/0803.0929.pdf, 2011.

[SWZ17] Zhao Song, David P Woodruff, and Peilin Zhong. Low rank approximation with entrywise $\ell_{1}$-norm error. In Proceedings of the 49th Annual Symposium on the Theory of Computing (STOC). ACM, https://arxiv.org/pdf/1611.00898, 2017.

[SWZ19] Zhao Song, David P Woodruff, and Peilin Zhong. Relative error tensor low rank approximation. In ACM-SIAM Symposium on Discrete Algorithms (SODA). https: //arxiv.org/pdf/1704.08246, 2019.

[Vai87] Pravin M Vaidya. An algorithm for linear programming which requires $o\left(\left((m+n) n^{2}+\right.\right.$ $\left.\left.(m+n)^{1.5} n\right) l\right)$ arithmetic operations. In 28th Annual IEEE Symposium on Foundations of Computer Science (FOCS), 1987.

[Vai89a] Pravin M Vaidya. A new algorithm for minimizing convex functions over convex sets. In 30th Annual IEEE Symposium on Foundations of Computer Science (FOCS), pages 338-343, 1989.

[Vai89b] Pravin M Vaidya. Speeding-up linear programming using fast matrix multiplication. In 30th Annual Symposium on Foundations of Computer Science (FOCS), pages 332337. IEEE, 1989.

[Vaz10] Vijay V Vazirani. Spending constraint utilities with applications to the adwords market. Mathematics of Operations Research, 35(2):458-478, 2010.

[Vég16] László A Végh. A strongly polynomial algorithm for a class of minimum-cost flow problems with separable convex objectives. volume 45(5), pages 1729-1761. https: //arxiv.org/pdf/1110.4882.pdf, 2016.

[Wan16] Zi Wang. A New Strongly Polynomial Algorithm for Computing Fisher Market Equilibria with Spending Constraint Utilities. PhD thesis, New York University, 2016.

[Wil12] Virginia Vassilevska Williams. Multiplying matrices faster than coppersmithwinograd. In Proceedings of the forty-fourth annual ACM symposium on Theory of computing (STOC), pages 887-898. ACM, 2012. 
[Woo49] Max A Woodbury. The stability of out-input matrices. Chicago, IL, 9, 1949.

[Woo50] Max A Woodbury. Inverting modified matrices. Memorandum report, 42(106):336, 1950.

[Ye08] Yinyu Ye. A path to the arrow-debreu competitive market equilibrium. Mathematical Programming, 111(1-2):315-348, 2008.

[YN76] David B Yudin and Arkadii S Nemirovski. Evaluation of the information complexity of mathematical programming problems. Ekonomika i Matematicheskie Metody, 12:128$142,1976$. 


\section{Preliminaries}

In this section we introduce the notions and tools used throughout this paper.

\subsection{Notations}

For a positive integer $n$, let $[n]$ denote the set $\{1,2, \cdots, n\}$. For any function $f$, we define $\widetilde{O}(f)$ to be $f \cdot \log ^{O(1)}(f)$. In addition to $O(\cdot)$ notation, for two functions $f, g$, we use the shorthand $f \lesssim g$ (resp. $\gtrsim$ ) to indicate that $f \leq C \cdot g$ (resp. $\geq$ ) for some absolute constant $C$.

For vector $x \in \mathbb{R}^{n}$, we use $\|x\|_{2}$ to denote its $\ell_{2}$ norm, we use $\|x\|_{1}$ to denote its $\ell_{1}$ norm, we use $\|x\|_{\infty}$ to denote its $\ell_{\infty}$ norm. For matrix $A \in \mathbb{R}^{m \times n}$, we use $\|A\|$ to denote the spectral norm of $A$, we use $\|A\|_{F}$ to denote its Frobenius norm $\left(\|A\|_{F}=\left(\sum_{i=1}^{m} \sum_{j=1}^{n} A_{i, j}^{2}\right)^{1 / 2}\right)$, we use $\|A\|_{1}$ to denotes its entry-wise $\ell_{1}$ norm $\|A\|_{1}=\sum_{i=1}^{m} \sum_{j=1}^{n}\left|A_{i, j}\right|$. We use $A^{\dagger}$ to denote its MooreâĂŞPenrose inverse. We use $A^{\top}$ denote the transpose of $A$.

For square matrix $A$, we use $\operatorname{tr}[A]$ to denote the trace of $A$. We say $A$ is positive-semidefinite (psd) if $A=A^{\top}$ and $x^{\top} A x \geq 0, \forall x \in \mathbb{R}^{n}$. We use $\succeq, \preceq$ to denote the semidefinite ordering, e.g. $A \succeq 0$ means that $A$ is psd.

For square full-rank matrix $A$, we use $A^{-1}$ to denote the inverse of $A$.

For vectors $a, b \in \mathbb{R}^{n}$ and accuracy parameter $\epsilon \in(0,1)$, we use $a \approx_{\epsilon} b$ to denote that $(1-\epsilon) b_{i} \leq$ $a_{i} \leq(1+\epsilon) b_{i}, \forall i \in[n]$. Similarly, for any scalar $t$, we use $a \approx_{\epsilon} t$ to denote that $(1-\epsilon) t \leq a_{i} \leq(1+\epsilon) t$ $\forall i \in[n]$.

\subsection{Operators}

Definition 3.1 (Operators $\tau$ and $\sigma$ ). Given a matrix $A \in \mathbb{R}^{m \times n}$ and a vector $v \in \mathbb{R}^{m}$, we define the leverage scores $\sigma(v)_{i}$ and (unnormalized) leverage scores $\tau(v)_{i}$ as follows

$$
\tau(v)_{i}=\left(A\left(A^{\top} V A\right)^{-1} A^{\top}\right)_{i, i} \text { and } \sigma(v)_{i}=\left(\sqrt{V} A\left(A^{\top} V A\right)^{-1} A^{\top} \sqrt{V}\right)_{i, i} .
$$

Definition 3.2 (Operators $M, Q$ and $P$ ). Given a matrix $A \in \mathbb{R}^{m \times n}$, we define three operators : $M: \mathbb{R}^{m} \rightarrow \mathbb{R}^{n \times n}, Q: \mathbb{R}^{m} \rightarrow \mathbb{R}^{m \times m}$ and $P: \mathbb{R}^{m} \rightarrow \mathbb{R}^{m \times m}$ such that for any vector $v \in \mathbb{R}^{m}$

$$
\begin{aligned}
M(v) & =A^{\top} V A \\
Q(v) & =A\left(A^{\top} V A\right)^{-1} A^{\top} \\
P(v) & =\sqrt{V} A\left(A^{\top} V A\right)^{-1} A^{\top} \sqrt{V} .
\end{aligned}
$$

\subsection{Different types of running time}

Definition 3.3 (Pseudo-polynomial time). An algorithm runs in pseudo-polynomial time if its running time is a polynomial in the length of the input (the number of bits required to represent it) and the numeric value of the input (the largest integer present in the input).

Definition 3.4 (Strongly-polynomial time). An algorithm runs in strongly polynomial time if (1) the number of operations in the arithmetic model of computation is bounded by a polynomial in the number of rational numbers in the input instance, and (2) the space used by the algorithm is bounded by a polynomial in the size of the input.

Definition 3.5 (Weakly-polynomial time). An algorithm runs in weakly-polynomial time if its running time is upper bounded by a polynomial in the size of the input, and it doesn't run in strongly polynomial time. 


\subsection{Basic results on matrices}

Fact 3.6 (Woodbury matrix identity, [Woo49, Woo50]). Given a square invertible $n \times n$ matrix A, an $n \times k$ matrix $U$ and $a k \times n$ matrix $V$, let $B$ be an $n \times n$ matrix such that $B=A+U C V$. Then, assuming $\left(I_{k}+V A^{-1} U\right)$ is invertible, we have

$$
B^{-1}=A^{-1}-A^{-1} U\left(C^{-1}+V A^{-1} U\right)^{-1} V A^{-1} .
$$

\subsection{Fast matrix multiplication}

Definition 3.7 (Matrix multiplication time). For any $n, r>0$, denote $\mathcal{T}_{\text {mat }}(n, n, r)$ the time to compute the multiplication of an $n \times n$ matrix and an $n \times r$ matrix.

Theorem 3.8 (Fast matrix multiplication). We have the following upper bound on $\mathcal{T}_{\text {mat }}(n, n, r)$ :

1. [GU18]. For $r=n^{0.31}$, we have $\mathcal{T}_{\text {mat }}(n, n, r)=O\left(n^{2+o(1)}\right)$.

2. [Cop82]. For $r=n^{0.17}$, we have $\mathcal{T}_{\text {mat }}(n, n, r)=O\left(n^{2} \log ^{2} n\right)$.

3. [BD'6]. For $r=\log ^{c} n$ for any constant $c>0$, then we have $\mathcal{T}_{\text {mat }}(n, n, r)=O\left(n^{2}\right)$.

\subsection{Multiple variable polynomial interpolation}

We first state a one-variable version interpolation theorem.

Theorem 3.9 (One variable [IK94, Eq 10 in Page 331]). Given any $2 N$ times differentiable function $f: \mathbb{R} \rightarrow \mathbb{R}$. There exits $N$ points $s_{1}, \cdots, s_{N}$ and $N$ weights $\omega_{1}, \cdots, \omega_{N} \geq 0$ such that $\sum_{i} \omega_{i}=1$ and

$$
\left|\int_{0}^{1} f(t) \mathrm{d} t-\sum_{i=1}^{N} \omega_{i} f\left(s_{i}\right)\right| \leq O(1) \cdot \frac{M_{2 N}}{(2 N) ! 4^{N}}
$$

where

$$
M_{2 N}=\max _{t \in[0,1]}\left|\frac{\partial^{(2 N)} f}{\partial t^{(2 N)}}(t)\right|
$$

Now, we explain how to use one variable interpolation result (Theorem 3.9) to prove a multiple variable interpolation result (Theorem 3.10).

Theorem 3.10 ( $d$-variable case). Given a function $f: \mathbb{R}^{d} \rightarrow \mathbb{R}$ such that for any $j \in[d]$, for any $t_{1}, \cdots, t_{j-1}, t_{j+1}, \cdots t_{d} \in[0,1], f\left(t_{1}, \cdots, t_{j-1}, t_{j}, t_{j+1}, \cdots, t_{d}\right)$ is $N+1$ times differentiable respect to $t_{j}$. Then there exists $N$ points $s_{1}, \cdots, s_{N}$ and $N$ weights $\omega_{1}, \cdots, \omega_{N}$ such that

$$
\left|\int_{[0,1]^{d}} f(t) \mathrm{d} t-\sum_{i_{1}=1}^{N} \cdots \sum_{i_{d}=1}^{N} \omega_{i_{1}} \cdots \omega_{i_{d}} f\left(s_{i_{1}}, \cdots, s_{i_{d}}\right)\right| \leq \frac{1}{(2 N) ! 2^{2 N}} \cdot \sum_{j=1}^{d} \max _{t \in[0,1]^{d}}\left|\frac{\partial^{(2 N)} f}{\partial t_{j}^{(2 N)}}(t)\right| .
$$

Proof. Let $\tau$ denote

$$
\tau=\frac{1}{(2 N) ! 2^{2 N}}
$$


Using one variable theorem 3.9, we have that : for all $t_{1}, t_{2}, \cdots, t_{d-1} \in[0,1]$,

$$
\left|\int_{0}^{1} f\left(t_{[d-1]}, t_{d}\right) \mathrm{d} t_{d}-\sum_{i_{d}=1}^{N} \omega_{i_{d}} f\left(t_{[d-1]}, s_{i_{d}}\right)\right| \leq \tau \cdot \max _{t_{[d-1]} \in[0,1]} \max _{t_{d} \in[0,1]}\left|f_{t_{d}}^{(2 N)}(t)\right|
$$

To write recursive thing in an easy way, we let $g_{d}$ denote function $f$. We use $t_{[j]}$ to denote $t_{1}, t_{2}, \cdots, t_{j}$.

For each $j \in\{d-1, \cdots, 1\}$, we define function $g_{j}: \mathbb{R}^{j} \rightarrow \mathbb{R}$ such that

$$
g_{j}\left(t_{[j]}\right)=\sum_{i_{j+1}=1}^{N} \omega_{i_{j+1}} g_{j+1}\left(t_{[j]}, s_{i_{j+1}}\right)
$$

Let $g_{0}=\sum_{i_{1}=1}^{N} \omega_{i_{1}} g_{1}\left(s_{i_{1}}\right)$, we can also rewrite $g_{0}$ as $\int_{[0,1]^{0}} g_{0}\left(t_{[0]}\right) \mathrm{d} t_{[0]}$.

Finally, we want to bound

$$
\begin{aligned}
& \left|\int_{0}^{1} \cdots \int_{0}^{1} f\left(t_{1}, \cdots, t_{d}\right) \mathrm{d} t_{1} \cdots \mathrm{d} t_{d}-\sum_{i_{1}=0}^{N} \cdots \sum_{i_{d}=1}^{N} \omega_{s_{i}} f\left(s_{i_{1}}, \cdots, s_{i_{d}}\right)\right| \\
\leq & \sum_{j=1}^{d}\left|\int_{[0,1]^{j}} g_{j}\left(t_{[j]}\right) \mathrm{d} t_{[j]}-\int_{[0,1]^{j-1}} g_{j-1}\left(t_{[j-1]}\right) \mathrm{d} t_{[j-1]}\right| \\
\leq & \tau \sum_{j=1}^{d} \max _{t \in[0,1]^{d}}\left|\frac{\partial^{(2 N)} f}{\partial t_{j}^{(2 N)}(t)}\right|,
\end{aligned}
$$

where the last step follows by Claim 3.11.

Claim 3.11 (Bounding the difference between $j$-th term and $j$-1-th term). For each $j \in[d]$,

$$
\left|\int_{[0,1]^{j}} g_{j}\left(t_{[j]}\right) \mathrm{d} t_{[j]}-\int_{[0,1]^{j-1}} g_{j-1}\left(t_{[j-1]}\right) \mathrm{d} t_{[j-1]}\right| \leq \tau \cdot \max _{t \in[0,1]^{d}}\left|\frac{\partial^{(2 N)} f}{\partial t_{j}^{(2 N)}(t)}\right| .
$$

Proof. First, using one variable theorem 3.9, we can upper bound

$$
\begin{aligned}
&\left|g_{j}\left(t_{[j-1]}, t_{j}\right) \mathrm{d} t_{j}-\sum_{i_{j}=1}^{N} \omega_{i_{j}} g_{j}\left(t_{[j-1]}, s_{i_{j}}\right)\right| \\
& \leq \tau \cdot \max _{t_{[j-1]} \in[0,1]^{j-1}} \max _{t_{j} \in[0,1]}\left|g_{j, t_{j}}^{(N+1)}\left(t_{[j]}\right)\right| \\
& \leq \cdots \\
& \leq \tau \cdot \max _{t_{[j-1]} \in[0,1]^{j-1}} \max _{t_{j} \in[0,1]} \max _{[d] \backslash[j]} \in[0,1]^{d-j} \\
&
\end{aligned}
$$

where the last step follows by re-using $\omega_{i}>0$ and $\sum_{i=1}^{N} \omega_{i}=1$. 
We have

$$
\begin{aligned}
& \left|\int_{[0,1]^{j}} g_{j}\left(t_{[j]}\right) \mathrm{d} t_{[j]}-\int_{[0,1]^{j-1}} g_{j-1}\left(t_{[j-1]}\right) \mathrm{d} t_{[j-1]}\right| \\
= & \left|\int_{[0,1]^{j}} g_{j}\left(t_{[j]}\right) \mathrm{d} t_{[j]}-\int_{[0,1]^{j-1}} \sum_{i_{j}=1}^{N} \omega_{i_{j}} g_{j}\left(t_{[j-1]}, s_{i_{j}}\right) \mathrm{d} t_{[j-1]}\right| \\
= & \left|\int_{[0,1]^{j-1}}\left(g_{j}\left(t_{[j-1]}, t_{j}\right) \mathrm{d} t_{j}-\sum_{i_{j}=1}^{N} \omega_{i_{j}} g_{j}\left(t_{[j-1]}, s_{i_{j}}\right)\right) \mathrm{d} t_{[j-1]}\right| \\
\leq & \int_{[0,1]^{j-1}}\left|g_{j}\left(t_{[j-1]}, t_{j}\right) \mathrm{d} t_{j}-\sum_{i_{j}=1}^{N} \omega_{i_{j}} g_{j}\left(t_{[j-1]}, s_{i_{j}}\right)\right| \mathrm{d} t_{[j-1]} \\
\leq & \tau \cdot \max _{t_{[j-1]} \in[0,1]^{j-1} \max _{t_{j} \in[0,1]} \max _{[d] \backslash[j]} \in[0,1]^{d-j}}\left|f_{t_{j}}^{(2 N)}\left(t_{[j-1]}, t_{j}, t_{[d] \backslash[j]}\right)\right| \\
= & \tau \cdot \max _{t \in[0,1]^{d}}\left|\frac{\partial^{(2 N)} f}{\partial t_{j}^{(2 N)}(t)}\right| .
\end{aligned}
$$




\section{Perturbed Volumetric Center Cutting Plane Method}

Recall that the feasibility problem is defined as follows.

Feasibility Problem: Given a separation oracle for a set $K$ contained in a box of radius $R$ either find a point $x \in K$ or prove that $K$ does not contain a ball of radius $\epsilon$.

Let $K \subset \mathbb{R}^{n}$ be a convex set that is contained in a box of radius $R$ and equipped with a separation oracle. To solve the feasibility problem, a cutting plane method iteratively refines a feasible region $\Omega$ and a query point $z$. In each iteration, $z$ is the input to the separation oracle which then either certifies $z \in K$ or produces a hyperplane $a^{\top} x=b$ separating $z$ from $K$. The algorithm then refines $\Omega$ and computes the next query point $z^{\text {(new) }}$ from the new separating hyperplane and any past information.

At a high level, Vaidya's method heavily employs leverage scores to decide which hyperplane to add or to drop from the feasible region. The main innovation behind our faster implementation is a data structure that maintains an estimate of the leverage scores in amortized $O\left(n^{2}\right)$. This removes the bottleneck in Vaidya's algorithm and yields the following result.

Theorem 4.1. Given a separation oracle for a convex set $K \subset \mathbb{R}^{n}$ that is contained in a box of radius $R$ and a parameter $\epsilon>0$, there is a cutting plane method that either computes a point in $K$ or proves that $K$ does not contain a ball of radius $\epsilon$ in $O\left(\left(n \mathrm{SO}+n^{3}\right) \log (\kappa)\right)$ time, where SO is the complexity of the separation oracle and $\kappa=n R / \epsilon$.

Since the proof of the validity of Vaidya's method for our result is mostly a perturbed version of his analysis, we defer the proof of Theorem 4.1 to Section A. 


\section{Main Data Structure for Leverage Score Maintenance}

In this section, we present our main data structure for leverage score maintenance that achieves an amortized $O\left(n^{2}\right)$ time per update. Combined with Theorem 4.1 in Section 4, our main data structure implies a faster $O\left(n \mathrm{SO} \log (\kappa)+n^{3} \log (\kappa)\right)$ time cutting plane method. Our main data structure further uses the simple leverage score maintenance data structure in Section 7 and the complicated leverage score maintenance data structure in Section 8.

Theorem 5.1 (Leverage score maintenance). Given an initial matrix $A \in \mathbb{R}^{m \times n}$ with $m=O(n)$, initial weight $w \in \mathbb{R}_{+}^{m}$. Then for any constant $c>0$, there is a randomized data structure (Algorithm 1) that approximately maintains the leverage scores

$$
\sigma_{i}(w)=\left(\sqrt{W} A\left(A^{\top} W A\right)^{-1} A^{\top} \sqrt{W}\right)_{i, i}
$$

for positive diagonal matrices $W=\operatorname{diag}(w)$ through the following operations:

1. $\operatorname{InIT}(A, w)$ : takes $O\left(n^{\omega+o(1)}\right)$ time to intialize the data structure.

2. UPDATE(act): updates the data structure for the single update act.

3. QUeRY () : takes $O(n)$ time to output a vector $\widetilde{\sigma} \in \mathbb{R}^{m}$.

Moreover, if the sequence of $K$ updates $\left(A^{(k)} \in \mathbb{R}^{m^{(k)} \times n}, w^{(k)} \in \mathbb{R}_{+}^{m^{(k)}}\right)$ with $m^{(k)}=O(n)$ satisfies that each update $k$ is one of the following:

1. Insertion (resp. deletion) of row a with weight $w_{a}$ into (resp. from) $\left(A^{(k-1)}, w^{(k-1)}\right)$ that satisfies

$$
w_{a} a a^{\top} \preceq 0.01\left(A^{(k-1)}\right)^{\top} W^{(k-1)} A^{(k-1)} .
$$

2. Update $w^{(k-1)} \in \mathbb{R}_{+}^{m^{(k-1)}}$ to $w^{(k)} \in \mathbb{R}_{+}^{m^{(k)}}$ such that $m^{(k-1)}=m^{(k)}$ and that

$$
\left\|\log \left(w^{(k)}\right)-\log \left(w^{(k-1)}\right)\right\|_{2} \leq 0.01,
$$

then the function UPDATE(act) takes an amortized $O\left(n^{2}\right)$ time, and the vector $\widetilde{\sigma}^{(k)}$ output by QueRY() at each step $k \in[K]$ satisfies that

$$
\left\|\widetilde{\sigma}^{(k)}-\sigma\left(w^{(k)}\right)\right\|_{2} \leq O\left(1 / \log ^{c}(n)\right) .
$$

The proof of Theorem 5.1 relies on the data structures in Sections 6, 7 and 8 .

Proof of Theorem 5.1. We first prove the running time upper bound of the different functions. Notice that the running time upper bound for $\operatorname{InIT}(A, w)$, and $\operatorname{QueRY}()$ are immediate corollaries of Theorem 7.2 and 8.1. We prove running time upper bound for the function UPDATE(act) in the following. In particular, we show that the inner, middle and outer phases all run in amortized $O\left(n^{2}\right)$ time, and the amortized time to restart the data structure in Step 29 is $O\left(n^{2}\right)$.

We start by analyzing the running time of the inner phase. Since each call to Update(act) makes one call of UPDATE(act) with act having a single action act, it follows from Theorem 7.2 that the inner phase makes one call to PM.UPDATE and takes extra time at most $O\left(n^{2}\right)$. Since we choose our parameter as $\epsilon_{\text {inn }}=1 / \log ^{25}(n)$ (see Table 5), it then follows from Theorem B.4 with 
$C=O(1)$ that the amortized time per call to PM.UPDATE is $O\left(n^{2}\right)$. Therefore, the inner phase runs in amortized time $O\left(n^{2}\right)$.

Next we analyze the running time of the middle phase. Notice that each middle update happens once every $T_{\mathrm{inn}}=\log ^{10}(n) \leq n^{0.08}$ calls to UpdATE(act), so Assumption 7.1 holds for the middle update in Step 23 of Algorithm 1. It then follows from Theorem 7.2 that for every $T_{\text {inn }}$ calls to the function UPDATE(act), the middle phase makes one call to PM.UPDATE and takes extra time at most $O\left(n^{2} T_{\text {inn }}\right)$. Since we choose $\epsilon_{\text {mid }}=1 / n^{0.08}$, it follows from Theorem B.2 with $C=T_{\text {inn }}=\log ^{10}(n)$ that the time for one call to PM.UPDATE is $O\left(n^{2} \log ^{2}(n)\right)$. Amortized over the $T_{\text {inn }}=\log ^{10}(n)$ calls to the function UPDATE(act), the amortized running time for the middle phase is thus $O\left(n^{2}\right)$.

Finally we analyze the running time of the outer phase. Each outer update happens once every $T_{\text {inn }} \cdot T_{\text {mid }}=n^{0.01} \log ^{10}(n)$ calls to UPDATE(act), so Assumption 7.1 holds for the outer update in Step 26 of Algorithm 1. It then follows from Theorem 8.1 that for every $T_{\text {inn }} \cdot T_{\text {mid }}$ calls to the function UPdATE(act), the outer phase makes $O(N)=O\left(\log ^{2}(n)\right)$ calls to PM.UPDATE and takes extra time at most

$$
O\left(n^{2} \cdot T_{\mathrm{inn}} \cdot T_{\mathrm{mid}}+T\left(n, n, r_{\mathrm{out}}\right) \cdot N^{3} \cdot \log (n)\right)=O\left(n^{2} \cdot T_{\mathrm{inn}} \cdot T_{\mathrm{mid}}+n^{2+o(1)} \log ^{7}(n)\right) .
$$

Since we choose $\epsilon_{\text {out }}=1 / n^{0.1}$, it follows from Theorem B.2 with $C=T_{\text {inn }} \cdot T_{\text {mid }}$ that the time for $O(N)$ calls to PM.update is $O\left(n^{2+o(1)}\right)$. Amortized over the $T_{\mathrm{inn}} \cdot T_{\operatorname{mid}}=n^{0.01} \log ^{10}(n)$ calls to the function UPDATE(act), the amortized running time for the outer phase is again $O\left(n^{2}\right)$.

It remains to upper bound the amortized time to restart the data structure due to Step 29. We note that restarting takes time $O\left(n^{\omega+o(1)}\right)$ every $T_{\mathrm{inn}} \cdot T_{\mathrm{mid}} \cdot T_{\text {out }}=\Omega\left(n^{\omega-1.99}\right)$ calls to the function $\operatorname{UPDATE}\left(\right.$ act). It follows that the amortized time to restart the data structure is $O\left(n^{\omega+o(1)}\right) /\left(T_{\mathrm{inn}}\right.$. $\left.T_{\text {mid }} \cdot T_{\text {out }}\right)=O\left(n^{2}\right)$. This finishes the running time analysis.

Now we proceed to prove the error guarantee given in the second part of the theorem. We only prove (1) for $c=15$ as it's easy to adjust the parameter settings in Table 5 to achieve the guarantee for any constant $c>0$. By Theorem 7.2, the $\ell_{2}$-error of the inner phase is $O\left(\epsilon_{\text {inn }}\right)=O\left(\log ^{-25}(n)\right)$ per call to $\operatorname{UpdATE}(\mathrm{act})$ and the inner phase runs for at most $T_{\mathrm{inn}}=\log ^{10}(n)$ steps before its estimate gets refined by the estimate of the middle or outer phases (Step 37 and 33). Therefore, the error introduced by the inner phase is at most $O\left(\epsilon_{\mathrm{inn}}\right) \cdot T_{\mathrm{inn}}=O\left(\log ^{-15}(n)\right)$. For the middle phase, it follows from Theorem 7.2 that the $\ell_{2}$-error introduced by each middle step is at most $O\left(\left(\epsilon_{\text {mid }}+T_{\text {inn }} \cdot n^{-0.08}\right) \cdot T_{\text {inn }}\right) \leq n^{-0.07}$. Since the middle phase run for at most $T_{\text {mid }}=n^{0.01}$ steps before its estimate gets refined by the estimate of the outer phase (Step 34), the error of the middle phase is at most $n^{-0.07} \cdot n^{0.01}=n^{-0.06}$. For the outer phase, the error in Theorem 8.1 is negligible so it suffices to bound the variance. From Theorem 8.1, the variance in each outer step is $O\left(N^{3} / r_{\text {out }}\right) \cdot \epsilon_{\text {out }}^{2} \cdot\left(T_{\text {inn }} \cdot T_{\text {mid }}\right)^{2} \leq O\left(n^{-0.48}\right)$. Since the outer phase run for at most $T_{\text {out }}=n^{\omega-2}$ steps before the data structure gets restarted, the variance accumulated in the $T_{\text {out }}$ steps is at most $O\left(n^{-0.1}\right)$. Therefore, we have $\left\|\widetilde{\sigma}^{(k)}-\sigma\left(w^{(k)}\right)\right\|_{2} \leq O\left(\log ^{-15}(n)\right)$ for each $k \in[K]$. This completes the proof of Theorem 5.1. 


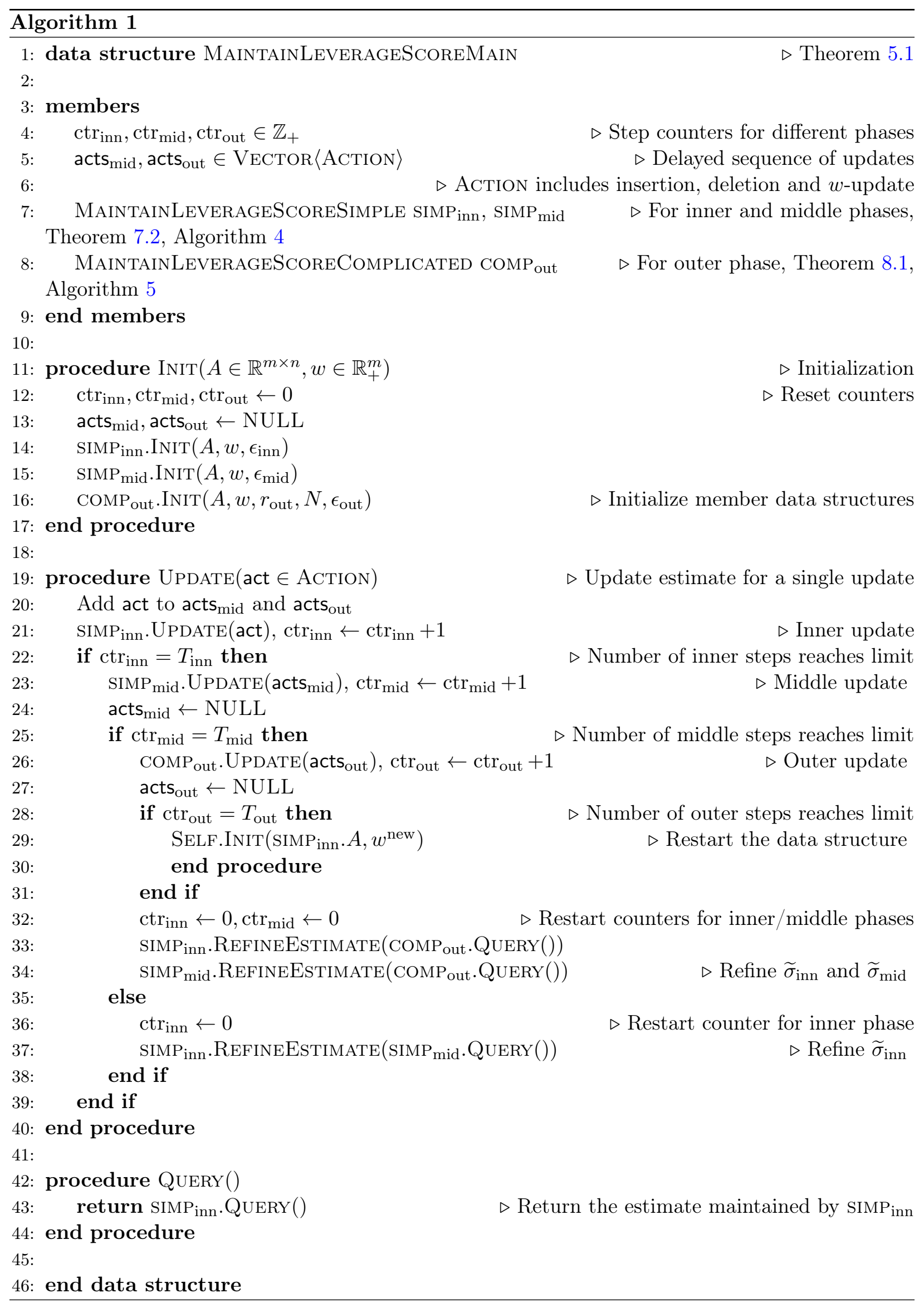




\begin{tabular}{|l|l|l|}
\hline Parameter & Value & Description \\
\hline$T_{\text {inn }}$ & $\log ^{10} n$ & Number of inner steps per middle step \\
\hline$T_{\text {mid }}$ & $n^{0.01}$ & Number of middle step per outer step \\
\hline$T_{\text {out }}$ & $n^{\omega-2}$ & Number of outer step before re-computing \\
\hline$r_{\text {out }}$ & $n^{0.31}$ & Matrix multiplication dimension for outer step \\
\hline$\epsilon_{\text {inn }}$ & $1 / \log ^{25} n$ & Inner step projection maintenance error \\
\hline$\epsilon_{\text {mid }}$ & $1 / n^{0.08}$ & Middle step projection maintenance error \\
\hline$\epsilon_{\text {out }}$ & $1 / n^{0.1}$ & Outer step projection maintenance error \\
\hline$N$ & $100 \log ^{2} n$ & Number of discrete sampling points in an outer step \\
\hline
\end{tabular}

Table 5: Values of parameters used in the main data structure for leverage score maintenance in Algorithm 1.

\section{Batched Low Rank Update}

\subsection{Simple low rank update}

In this section, we show how to maintain leverage score under low rank update. First, we start with a preconditioning lemma showing that given two spectrally similar matrix, we can invert one matrix faster by knowing the inverse of the other matrix.

Lemma 6.1. Given $n \times n$ PSD matrices $A$ and $M$ such that $0 \preceq M \preceq A \preceq \kappa \cdot M$. For any integer $t \geq 1$ such that $\kappa(1-1 / \kappa)^{t+1}<1$, we have

$$
f(M, t) \preceq A^{-1} \preceq \frac{1}{1-\kappa(1-1 / \kappa)^{t+1}} \cdot f(M, t)
$$

where $f: \mathbb{R}^{n \times n} \times \mathbb{N} \rightarrow \mathbb{R}^{n \times n}$ is defined as

$$
f(M, t)=\frac{1}{\kappa} M^{-1} \sum_{i=0}^{t}\left(I-\frac{1}{\kappa} A M^{-1}\right)^{i} .
$$

Furthermore, if $M^{-1}$ and $A$ are given explicitly, for any $V \in \mathbb{R}^{n \times r}$, then we can compute $f(M, t) \cdot V$ in $O\left(\mathcal{T}_{\text {mat }}(n, n, r) \cdot t\right)$ time.

Proof. Note that

$$
\begin{aligned}
A^{-1} & =M^{-\frac{1}{2}}\left(M^{-\frac{1}{2}} A M^{-\frac{1}{2}}\right)^{-1} M^{-\frac{1}{2}} \\
& =\frac{1}{\kappa} M^{-\frac{1}{2}}\left(I-\left(I-\frac{1}{\kappa} M^{-\frac{1}{2}} A M^{-\frac{1}{2}}\right)\right)^{-1} M^{-\frac{1}{2}} .
\end{aligned}
$$

Using

$$
0 \preceq I-(1 / \kappa) \cdot M^{-\frac{1}{2}} A M^{-\frac{1}{2}} \preceq(1-1 / \kappa) \cdot I
$$

and

$$
\sum_{i=0}^{t} x^{i} \leq(1-x)^{-1} \leq \sum_{i=0}^{t} x^{i}+\kappa(1-1 / \kappa)^{t+1} \quad \forall x \in[0,1-1 / \kappa]
$$


we have

$$
\begin{aligned}
\sum_{i=0}^{t}\left(I-\frac{1}{\kappa} M^{-\frac{1}{2}} A M^{-\frac{1}{2}}\right)^{i} & \preceq\left(I-\left(I-\frac{1}{\kappa} M^{-\frac{1}{2}} A M^{-\frac{1}{2}}\right)\right)^{-1} \\
& \preceq \sum_{i=0}^{N}\left(I-\frac{1}{\kappa} M^{-\frac{1}{2}} A M^{-\frac{1}{2}}\right)^{i}+\kappa \cdot(1-1 / \kappa)^{t+1} I
\end{aligned}
$$

Multiplying $\frac{1}{\kappa} M^{-1}$ on both sides of the above equation with a formula $A^{-1}$ (Eq. (2)), we get

$$
\frac{1}{\kappa} M^{-1} \sum_{i=0}^{t}\left(I-\frac{1}{\kappa} M^{-\frac{1}{2}} A M^{-\frac{1}{2}}\right)^{i} \preceq A^{-1} \preceq \frac{1}{\kappa} M^{-1} \sum_{i=0}^{t}\left(I-\frac{1}{\kappa} M^{-\frac{1}{2}} A M^{-\frac{1}{2}}\right)^{i}+(1-1 / \kappa)^{t+1} M^{-1}
$$

Using the definition of $f(M, t)$, we have that

$$
f(M, t) \preceq A^{-1} \preceq f(M, t)+(1-1 / \kappa)^{t+1} M^{-1} .
$$

Using $M^{-1} \preceq \kappa A^{-1}$, we have

$$
f(M, t) \preceq A^{-1} \preceq f(M, t)+\kappa \cdot(1-1 / \kappa)^{t+1} A^{-1} .
$$

The result follows from some rearranging.

Now, we show how to do leverage score update under monotone updates.

Lemma 6.2. Given a matrix $A \in \mathbb{R}^{m \times n}$, non-negative weights $w, w^{\text {new }} \in \mathbb{R}^{m}$ such that $w^{\text {new }} \geq w$ with $m=O(n)$ and $\left\|w^{\text {new }}-w\right\|_{0}=k$. Let $\beta>1$ denote the parameter such that $A^{\top} W^{\text {new }} A \preceq$ $\beta \cdot A^{\top} W A$. Given some explicit matrix $U \in \mathbb{R}^{n \times n}$ and $U^{\text {new }} \in \mathbb{R}^{n \times n}$ such that

$$
\begin{aligned}
U^{-1} & \preceq A^{\top} W A \preceq(1+1 / \log n) U^{-1}, \\
\left(U^{\text {new }}\right)^{-1} & \preceq A^{\top} W^{\text {new }} A \preceq(1+1 / \log n)\left(U^{\text {new }}\right)^{-1} .
\end{aligned}
$$

Then, for any $\epsilon \in(0,1 / 2)$, we can output a vector $c \in \mathbb{R}^{m}$ such that

$$
\left\|c-\left(\sigma\left(w^{\text {new }}\right)-\sigma(w)\right)\right\|_{2} \leq \epsilon
$$

in time

$$
O\left(\mathcal{T}_{\text {mat }}(n, n, k) \cdot\left(1+\log _{\log (n)}(\beta k / \epsilon)\right)\right) .
$$

The same statement holds for decreasing $w$, namely $w^{\text {new }} \leq w$, with $A^{\top} W^{\text {new }} A \succeq \beta^{-1} A^{\top} W A$.

Proof. We note that the decreasing case follows from the increasing case by swapping $w$ and $w^{\text {new }}$. Hence, we focus on the increasing case. Using Lemma 6.1, we can construct $U_{\epsilon}$ such that $U_{\epsilon}$ is low-degree polynomial of $U$ and $A^{\top} W A$ and that

$$
U_{\epsilon} \preceq\left(A^{\top} W A\right)^{-1} \preceq(1+\epsilon) U_{\epsilon} .
$$

We define $U_{\epsilon}^{\text {new }}$ similarly. We will show how to use $U_{\epsilon}$ and $U_{\epsilon}^{\text {new }}$ to approximate $\sigma\left(w^{\text {new }}\right)-\sigma(w)$. First, we note that

$$
\sigma\left(w^{\text {new }}\right)-\sigma(w)=\left(w^{\text {new }}-w\right) \cdot \tau\left(w^{\text {new }}\right)+w \cdot\left(\tau\left(w^{\text {new }}\right)-\tau(w)\right)
$$


We define $c_{i}, c_{1, i}, c_{2, i}$ as follows:

$$
\begin{aligned}
c_{i} & =\sigma\left(w^{\text {new }}\right)_{i}-\sigma(w)_{i} \\
& =\underbrace{\left(w^{\text {new }}-w\right)_{i} \cdot \tau\left(w^{\text {new }}\right)_{i}}_{c_{1, i}}+\underbrace{w_{i} \cdot\left(\tau\left(w^{\text {new }}\right)-\tau(w)\right)_{i}}_{c_{2, i}}
\end{aligned}
$$

We estimate the right hand side by

$$
\widetilde{c}_{i}:=\underbrace{\left(w^{\text {new }}-w\right)_{i} \cdot\left(A U_{\widetilde{\epsilon}}^{\text {new }} A^{\top}\right)_{i, i}}_{\widetilde{c}_{1, i}}+\underbrace{w_{i} \cdot\left(A\left(\left(U_{\widetilde{\epsilon}}^{-1}+\Delta\right)^{-1}-U_{\widetilde{\epsilon}}\right) A^{\top}\right)_{i, i}}_{\widetilde{c}_{2, i}}
$$

where $\Delta=A^{\top} W^{\text {new }} A-A^{\top} W A$ and $\widetilde{\epsilon}=\frac{\epsilon}{3 \beta \sqrt{k}}$.

Cost of computing $\widetilde{c}$ For the first term in (5). We note that $w^{\text {new }}-w$ has only $k$ non-zeros. Hence, we only need to compute $A U_{\widetilde{\epsilon}}^{\text {new }} A^{\top}$ on $k$ of the diagonals. Let $A_{S} \in \mathbb{R}^{k \times n}$ where each rows of $A$ that $w^{\text {new }} \neq w$. Then, we can compute the $k$ of the diagonals via the whole matrix

$$
A_{S} U_{\widetilde{\epsilon}}^{\text {new }} A_{S}^{\top} \text {. }
$$

Lemma 6.1 shows that $U_{\widetilde{\epsilon}}^{\text {new }}$ has $O\left(1+\frac{\log \widetilde{\epsilon}}{\log \log (n)}\right)$ terms and that it takes

$$
O\left(\mathcal{T}_{\text {mat }}(n, n, k) \cdot\left(1+\frac{\log \widetilde{\epsilon}}{\log \log (n)}\right)\right)
$$

to compute $U_{\widetilde{\epsilon}}^{\text {new }} A_{S}^{\top}$. Finally, it takes extra $\mathcal{T}_{\text {mat }}(k, n, k)=O\left(\mathcal{T}_{\text {mat }}(n, n, k)\right)$ to do the left multiplication on $A_{S}$.

For the second term in (5). Woodbury matrix identity shows that

$$
\left(U_{\widetilde{\epsilon}}^{-1}+\Delta\right)^{-1}-U_{\widetilde{\epsilon}}=U_{\widetilde{\epsilon}} A_{S}^{\top}\left(\Delta_{W}^{-1}+A_{S} U_{\widetilde{\epsilon}} A_{S}^{\top}\right)^{-1} A_{S} U_{\widetilde{\epsilon}}
$$

where $\Delta_{W} \in \mathbb{R}^{k \times k}$ be $W^{\text {new }}-W$ restricted on non-zeros. By same argument above, we can compute $A_{S} U_{\widetilde{\epsilon}} A_{S}^{\top}$ in

$$
O\left(\mathcal{T}_{\text {mat }}(n, n, k) \cdot\left(1+\frac{\log \widetilde{\epsilon}}{\log \log (n)}\right)\right)
$$

time.

Then, we can compute $\left(\Delta_{W}^{-1}+A_{S} U_{\widetilde{\epsilon}} A_{S}^{\top}\right)^{-1}$ in $\mathcal{T}_{\text {mat }}(k, k, k)=O\left(\mathcal{T}_{\text {mat }}(n, n, k)\right)$ time. Then, we compute

$$
A U_{\widetilde{\epsilon}} A_{S}^{\top}\left(\Delta_{W}^{-1}+A_{S} U_{\widetilde{\epsilon}} A_{S}^{\top}\right)^{-1} \quad \text { in time } \mathcal{T}_{\text {mat }}(m, n, k)=O\left(\mathcal{T}_{\text {mat }}(n, n, k)\right)
$$

and

$$
A_{S} U_{\widetilde{\epsilon}}=\left(U_{\widetilde{\epsilon}} A_{S}^{\top}\right)^{\top} \quad \text { in time } O\left(\mathcal{T}_{\text {mat }}(n, n, k) \cdot\left(1+\frac{\log \widetilde{\epsilon}}{\log \log (n)}\right)\right)
$$

Now, we multiple the two terms above together in time $\mathcal{T}_{\text {mat }}(n, k, n)=O\left(\mathcal{T}_{\text {mat }}(n, n, k)\right)$. Hence, the total time is

$$
O\left(\mathcal{T}_{\text {mat }}(n, n, k) \cdot\left(1+\frac{\log \tilde{\epsilon}}{\log \log (n)}\right)\right)=O\left(\mathcal{T}_{\text {mat }}(n, n, k)\left(1+\log _{\log (n)}(\beta k / \epsilon)\right)\right) .
$$


The accuracy of $\widetilde{c}$ For the first term in $\widetilde{c}$, we have

$$
A\left(A^{\top} W^{\text {new }} A\right)^{-1} A^{\top} \preceq A U_{\widetilde{\epsilon}}^{\text {new }} A^{\top} \preceq(1+\widetilde{\epsilon}) \cdot A\left(A^{\top} W^{\text {new }} A\right)^{-1} A^{\top} .
$$

Hence, we have

$$
\begin{aligned}
\left|\widetilde{c}_{1, i}-c_{1, i}\right| & =\left|\left(w^{\text {new }}-w\right)_{i}\left(A U_{\widetilde{\epsilon}}^{\text {new }} A^{\top}-A\left(A^{\top} W^{\text {new }} A\right)^{-1} A^{\top}\right)_{i, i}\right| \\
& \leq \widetilde{\epsilon} \cdot\left|w_{i}^{\text {new }}-w_{i}\right| \cdot\left(A\left(A^{\top} W^{\text {new }} A\right)^{-1} A^{\top}\right)_{i, i} \\
& \leq \widetilde{\epsilon} \cdot w_{i}^{\text {new }} \cdot\left(A\left(A^{\top} W^{\text {new }} A\right)^{-1} A^{\top}\right)_{i, i} \\
& \leq \widetilde{\epsilon}
\end{aligned}
$$

where we used that $w^{\text {new }} \geq w$ in the second inequality and leverage score is upper bounded by 1 in the last equality. Hence the $\ell_{\infty}$ norm error of the first term is given by $\widetilde{\epsilon}$. Hence, the $\ell_{2}$ norm error is given by

$$
\left\|\widetilde{c}_{1}-c_{1}\right\|_{2} \leq \sqrt{k} \cdot \widetilde{\epsilon}
$$

For the second term in $\widetilde{c}$, we have that the error is given by

$$
\widetilde{c}_{2, i}-c_{2, i}=w_{i} \cdot\left(A\left(\left(U_{\widetilde{\epsilon}}^{-1}+\Delta\right)^{-1}-U_{\widetilde{\epsilon}}\right) A^{\top}\right)_{i, i}-w_{i} \cdot\left(A\left((M+\Delta)^{-1}-M^{-1}\right) A^{\top}\right)_{i, i}
$$

where $M=A^{\top} W A$. To simplify the notation, we define $M_{t, s}=M+t \cdot \Delta_{2}+s \cdot \Delta$ where $\Delta_{2}=U_{\widetilde{\epsilon}}^{-1}-M$. Then, the error term becomes

$$
\left(\sqrt{W} A\left(M_{1,1}^{-1}-M_{1,0}^{-1}-M_{0,1}^{-1}+M_{0,0}^{-1}\right) A^{\top} \sqrt{W}\right)_{i, i}
$$

Note that

$$
\begin{aligned}
& M_{1,1}^{-1}-M_{1,0}^{-1}-M_{0,1}^{-1}+M_{0,0}^{-1} \\
= & \int_{0}^{1} \int_{0}^{1} \frac{\mathrm{d}^{2}}{\mathrm{~d} t \mathrm{~d} s} M_{t, s}^{-1} \mathrm{~d} t \mathrm{~d} s \\
= & \int_{0}^{1} \int_{0}^{1} M_{t, s}^{-1} \Delta_{2} M_{t, s}^{-1} \Delta M_{t, s}^{-1}+M_{t, s}^{-1} \Delta M_{t, s}^{-1} \Delta_{2} M_{t, s}^{-1} \mathrm{~d} t \mathrm{~d} s
\end{aligned}
$$

Combining (7) and (8), we have that the $\ell_{2}$ norm error for the second term is bounded by

$$
\begin{aligned}
\left\|\widetilde{c}_{2}-c_{2}\right\|_{2} & \leq\left\|\int_{0}^{1} \int_{0}^{1} \sqrt{W} A\left(M_{t, s}^{-1} \Delta_{2} M_{t, s}^{-1} \Delta M_{t, s}^{-1}+M_{t, s}^{-1} \Delta M_{t, s}^{-1} \Delta_{2} M_{t, s}^{-1}\right) A^{\top} \sqrt{W} \mathrm{~d} t \mathrm{~d} s\right\|_{F} \\
& \leq 2 \max _{t \in[0,1], s \in[0,1]}\left\|\sqrt{W} A M_{t, s}^{-1} \Delta_{2} M_{t, s}^{-1} \Delta M_{t, s}^{-1} A^{\top} \sqrt{W}\right\|_{F} .
\end{aligned}
$$

To bound the Frobenius norm, we note that $\Delta \succeq 0$ by the assumption and that $\Delta_{2}=U_{\widetilde{\epsilon}}^{-1}-M \succeq 0$ (4). Hence, we have that $M_{t, s} \succeq M$ and

$$
\begin{aligned}
& \left\|\sqrt{W} A M_{t, s}^{-1} \Delta_{2} M_{t, s}^{-1} \Delta M_{t, s}^{-1} A^{\top} \sqrt{W}\right\|_{F}^{2} \\
= & \operatorname{tr}\left[A^{\top} W A M_{t, s}^{-1} \Delta_{2} M_{t, s}^{-1} \Delta M_{t, s}^{-1} A^{\top} W A M_{t, s}^{-1} \Delta M_{t, s}^{-1} \Delta_{2} M_{t, s}^{-1}\right] \\
= & \operatorname{tr}\left[M^{\frac{1}{2}} M_{t, s}^{-1} \Delta_{2} M_{t, s}^{-1} \Delta M_{t, s}^{-1} M M_{t, s}^{-1} \Delta M_{t, s}^{-1} \Delta_{2} M_{t, s}^{-1} M^{\frac{1}{2}}\right] \\
\leq & \operatorname{tr}\left[M^{\frac{1}{2}} M_{t, s}^{-1} \Delta_{2} M_{t, s}^{-1} \Delta M_{t, s}^{-1} \Delta M_{t, s}^{-1} \Delta_{2} M_{t, s}^{-1} M^{\frac{1}{2}}\right] \\
= & \operatorname{tr}\left[M_{t, s}^{-\frac{1}{2}} \Delta M_{t, s}^{-1} \Delta_{2} M_{t, s}^{-1} M^{\frac{1}{2}} M^{\frac{1}{2}} M_{t, s}^{-1} \Delta_{2} M_{t, s}^{-1} \Delta M_{t, s}^{-\frac{1}{2}}\right] \\
\leq & \operatorname{tr}\left[M_{t, s}^{-\frac{1}{2}} \Delta M_{t, s}^{-1} \Delta_{2} M^{-1} \Delta_{2} M_{t, s}^{-1} \Delta M_{t, s}^{-\frac{1}{2}}\right] .
\end{aligned}
$$


(4) shows that $\Delta_{2} \preceq \widetilde{\epsilon} \cdot M$ and hence $\left(M^{-\frac{1}{2}} \Delta_{2} M^{-\frac{1}{2}}\right)^{2} \preceq \widetilde{\epsilon}^{2} \cdot I$. Continuing the last equation, we have

$$
\begin{aligned}
\left\|\sqrt{W} A M_{t, s}^{-1} \Delta_{2} M_{t, s}^{-1} \Delta M_{t, s}^{-1} A^{\top} \sqrt{W}\right\|_{F}^{2} & \leq \widetilde{\epsilon}^{2} \cdot \operatorname{tr}\left[M_{t, s}^{-\frac{1}{2}} \Delta M_{t, s}^{-1} M M_{t, s}^{-1} \Delta M_{t, s}^{-\frac{1}{2}}\right] \\
& \leq \widetilde{\epsilon}^{2} \cdot \operatorname{tr}\left[M_{t, s}^{-\frac{1}{2}} \Delta M_{t, s}^{-1} \Delta M_{t, s}^{-\frac{1}{2}}\right] \\
& \leq \widetilde{\epsilon}^{2} \cdot\left\|M^{-\frac{1}{2}} \Delta M^{-\frac{1}{2}}\right\|_{F}^{2} .
\end{aligned}
$$

Finally, using $\Delta$ has rank $k$, we have

$$
\begin{aligned}
\left\|\widetilde{c}_{2}-c_{2}\right\|_{2} & \leq 2 \widetilde{\epsilon} \cdot\left\|M^{-\frac{1}{2}} \Delta M^{-\frac{1}{2}}\right\|_{F} \\
& \leq 2 \widetilde{\epsilon} \cdot \sqrt{k}\left\|M^{-\frac{1}{2}} \Delta M^{-\frac{1}{2}}\right\| \\
& \leq 2 \widetilde{\epsilon} \cdot \beta \sqrt{k} .
\end{aligned}
$$

Combining (6) and (9), the total $\ell_{2}$ error is bounded by $2 \widetilde{\epsilon} \beta \sqrt{k}+\sqrt{k} \cdot \widetilde{\epsilon} \leq 3 \widetilde{\epsilon} \beta \sqrt{k}$. This explains the choice of $\widetilde{\epsilon}$.

\subsection{Batched low rank update}

When we are given a sequence of update and we need to compute the change of leverage score, we cannot apply Lemma 6.2 directly for the following reasons:

- The update in general involves both increasing some weight and decreasing some weight. This can be fixed by splitting the update into positive update and negative update. (See Step 1 in Algorithm 2)

- To get error $\frac{1}{n^{O(1)}}$, Lemma 6.2 takes at least $n^{2} \log (n)$ time. Hence, it is too costly to do a rank 1 update, which can happen if we have alternating positive and negative updates (since we can only apply Lemma 6.2 for a monotone change). This can be fixed by moving all positive/update and insert in the front of the update sequence. (See Step 2 in Algorithm 2)

- Our $\ell_{2}$ update are generally dense. This can be fixed by ignoring all small multiplicative update. (See Line 44 and 49 of Step 3 in Algorithm 2)

- In general, the matrices $A^{\top} W A$ can change by a factor of $e^{\Theta(T)}$ after $T$ iterations. So, Lemma 6.2 takes at least $\Omega\left(\mathcal{T}_{\text {mat }}(n, n, k) \frac{T}{\log \log (n)}\right)$ time. This forces us to do rank $k$ changes where $\mathcal{T}_{\text {mat }}(n, n, k) \leq n^{2} \log \log (n)$. Hence, for $\ell_{2}$ update, we need to handle coordinates with notso-small multiplicative updates separately. This can be fixed by cutting the update sequence into phases of length $L$ and noticing there are not too many coordinate that is changed by $e^{\Theta(L)}$ factor. (See the rest of Step 3 in Algorithm 2)

Given the above intuitions, Theorem 6.3 follows from multiple use of Lemma 6.2.

Theorem 6.3 (Batched Update of Leverage Score). Given an initial matrix $A \in \mathbb{R}^{m \times n}$, initial weight $w \in \mathbb{R}_{+}^{m}$ and a sequence of $T$ updates that belong to one of the following forms:

- Update: Update the weight to w

- Insert: Insert a row $a \in \mathbb{R}^{n}$ to the matrix $A \in \mathbb{R}^{m \times n}$ and weight $w_{a}$ to the vector $w$

- Delete: Delete a row $a \in \mathbb{R}^{n}$ from the matrix $A \in \mathbb{R}^{m \times n}$ and weight $w_{a}$ from the vector $w$ 
Let the sequence of matrices and vectors be $A^{(0)}, A^{(1)}, \cdots, A^{(T)} \in \mathbb{R}^{m \times n}$ and $w^{(0)}, w^{(1)}, \cdots, w^{(T)} \in$ $\mathbb{R}^{m}$. We assume that $T \leq n^{0.08}$, the maximum number of rows is bounded by $O(n)$, and that the changes satisfy

- Update: $\left\|\log w^{(k)}-\log w^{(k-1)}\right\|_{2} \leq 0.01$

- Insert/Delete: $w_{a} a a^{\top} \preceq 0.01 \cdot A^{(k-1)} W^{(k-1)} A^{(k-1)}$

Then, there is an algorithm (BATCHEDUPDATE in Algorithm 2 and 3) that finds a vector $v \in \mathbb{R}^{n}$ and a vector $c \in \mathbb{R}^{n}$ such that

$$
\left\|\log v-\log w^{(T)}\right\|_{\infty} \leq T \cdot n^{-0.08},\left\|\log v-\log w^{(T)}\right\|_{2} \leq 0.01 \cdot T
$$

and that

$$
\left\|c-\left(\sigma_{A^{(T)}}(v)-\sigma_{A^{(0)}}\left(w^{(0)}\right)\right)\right\|_{2} \leq 1 / n^{100} .
$$

in time $O\left(n^{2} T\right)$.

Proof.

Correctness Note that the algorithm 2 involves reducing the sequence $\left\{M^{(0, k)}\right\}$ to $\left\{M^{(1, k)}\right\}$ to $\left\{M^{(2, k)}\right\}$ and finally to $\left\{M^{(3, k)}\right\}$. All steps maintains the matrix at $k=0$. The first two steps maintains the matrix at the last step. For the last step, we only ignore the rows with multiplicative changes less than $\eta=n^{-0.08}$. Since there are at most $T / L=n^{0.08} / \log ^{3}(n)$ phases, the total accumulated multiplicative changes for one row is $T n^{-0.08}$. Therefore, we have

$$
M^{(3, \mathrm{END})}=\left(A^{(T)}\right)^{\top} V A^{(T)}
$$

for some $v$ such that $\left\|\log v-\log w^{(T)}\right\|_{\infty} \leq T n^{-0.08}$. Furthermore, we output the good enough approximation of $\sigma_{A^{(T)}}(v)-\sigma_{A^{(0)}}\left(w^{(0)}\right)=\sigma\left(M^{(3, \mathrm{END})}\right)-\sigma\left(M^{(3,0)}\right)$ where we abused the notation to use $\sigma(M)$ to denote the leverage score of the rows insides $M$. This is simply because we split the difference into

$$
\sigma\left(M^{(3, \mathrm{END})}\right)-\sigma\left(M^{(3,0)}\right)=\sum_{k=1}^{T} \sigma\left(M^{(3, k)}\right)-\sigma\left(M^{(3, k-1)}\right)
$$

and estimate each step with $\ell_{2}$ error $n^{-1000}$. There are at most $8 T$ many terms, so the total error is less than $n^{-100}$. (The number of terms can increases by at most a factor of 8 due to step 1 , step 2 and step 4.)

Ratio on $M$ within each phase By the assumptions on insert/delete and update, we see that $0.8 M^{(0, k-1)} \preceq M^{(0, k)} \preceq 1.2 M^{(0, k-1)}$ for all $k$. The first step clearly maintains this relation. We claim that the second step also maintains this relation. To see this, we let $\Delta^{(1, k)}=M^{(1, k+1)}-M^{(1, k)}$. By the relation, we have that

$$
-0.2 M^{(1, k)} \preceq \Delta^{(k)} \preceq 0.2 M^{(1, k)} .
$$

Note that step 2 simply permutes $\Delta^{(1, k)}$, namely, for each $k$ there is an unique $k^{\prime}$ such that $\Delta^{(1, k)}=$ $\Delta^{\left(2, k^{\prime}\right)}$. Finally, we note that $M^{(1, k)} \preceq M^{\left(2, k^{\prime}\right)}$ for all $k$ because $M^{\left(2, k^{\prime}\right)}$ is simply $M^{\left(2, k^{\prime}\right)}$ plus some 
PSD matrix (which due to some insert/positive update move into the sum or some delete/negative update removed from the sum). Hence, we still have the relation (10).

For the step 3, we should not expect the relation (10). However, we claim that within each phase, let $M^{(3, k)}$ and $M^{(3, \bar{k})}$ be two matrices in the phase. Then, we have that

$$
2^{-O(L)} M^{(3, \bar{k})} \preceq M^{(3, k)} \preceq 2^{O(L)} M^{(3, \bar{k})} .
$$

The proof for this is same for the phase with increasing $M$ and the phase with decreasing phase. Hence, we only discuss the first case. Say $k_{1}$ is the first matrix in the phase and $k_{2}$ is the last matrix in the phase. Note that the step 3 does not change the matrix $M^{\left(3, k_{1}\right)}$ and $M^{\left(3, k_{2}\right)}$ because we only swapping operation order within a phase. Hence by (10), we have

$$
M^{\left(3, k_{2}\right)}=M^{\left(2, k_{2}\right)} \preceq 2^{O(L)} M^{\left(2, k_{1}\right)}=2^{O(L)} M^{\left(3, k_{1}\right)} .
$$

Since all matrix in the phase is sandwiched between $M^{\left(3, k_{1}\right)}$ and $M^{\left(3, k_{2}\right)}$ (due to the monotonicity of the sequence of $M)$. Hence, we have (11).

Cost of Insert/Delete (Line 65) Now, we bound the runtime. We will show the each phase takes $O\left(n^{2} L\right)$ time. Since there are $O(T / L)$ phases, this shows that the total cost is $O\left(n^{2} T\right)$.

Each phase contains at most $L$ insert or delete. Hence, Line 65 involves estimating leverage score up to rank $L$ update. By (11), we know the matrix is changed by a $2^{O(L)}$ factor. Theorem 6.3 shows the cost is

$$
O\left(\mathcal{T}_{\text {mat }}(n, n, k)\left(1+\log _{\log (n)}(\beta k / \epsilon)\right)\right)
$$

where $\epsilon=n^{-1000}, \beta=2^{O(L)}$ and $k=O(L)$. By the choice of $L=\log ^{3}(n)$, we have $\mathcal{T}_{\text {mat }}(n, n, k)=n^{2}$ and hence the cost is $O\left(n^{2} L\right)$.

Cost of Large Update (Line 63) Each phase contains at most $L$ updates. By the assumption, each update change the $\ell_{2}$ norm of $\log w$ by at most $O(1)$. Note that this is maintains during all steps. Hence, after merging all updates into 1 , we have $\left\|\log w^{(k)}-\log w^{(k-1)}\right\|_{2}=O(L)$. By the construction of $w^{\left(k-\frac{1}{2}\right)}$, we have that $\left\|\log w^{(k)}-\log w^{\left(k-\frac{1}{2}\right)}\right\|_{2}=O(L)$ and that $\mid \log w_{i}^{\left(k-\frac{1}{2}\right)}-$ $\log w_{i}^{(k)} \mid$ is either 0 or at least $\log 2$ for all $i$. Hence the number of coordinates that is non-zero in $\log w^{(k)}-\log w^{\left(k-\frac{1}{2}\right)}$ is at most $O\left(L^{2}\right)$. Again, by (11), we know the matrix is changed by a $2^{O(L)}$ factor. Hence, Theorem 6.3 shows the cost is

$$
O\left(\mathcal{T}_{\text {mat }}(n, n, k)\left(1+\log _{\log (n)}(\beta k / \epsilon)\right)\right)
$$

where $\epsilon=n^{-1000}, \beta=2^{O(L)}$ and $k=O\left(L^{2}\right)$. By the choice of $L=\log ^{3}(n)$, we have $\mathcal{T}_{\text {mat }}(n, n, k)=$ $n^{2}$ and hence the cost is $O\left(n^{2} L\right)$.

Cost of Small Update (Line 62) Similar to above, we have that $\left\|\log w^{\left(k-\frac{1}{2}\right)}-\log w^{(k-1)}\right\|_{2}=$ $O(L)$. Furthermore, we have that $\left\|\log w^{\left(k-\frac{1}{2}\right)}-\log w^{(k-1)}\right\|_{\infty}=O(1)$. Due to Line 44 and 49 , we removed all rows with multiplicative changes less than $\eta$. Hence, the number of coordinates that is non-zero in $\log w^{\left(k-\frac{1}{2}\right)}-\log w^{(k-1)}$ is at most $O\left(L^{2} / \eta^{2}\right)$. Hence, Theorem 6.3 shows the cost is

$$
O\left(\mathcal{T}_{\text {mat }}(n, n, k)\left(1+\log _{\log (n)}(\beta k / \epsilon)\right)\right)
$$

where $\epsilon=n^{-1000}, \beta=O(1)$ and $k=O\left(L^{2} / \eta^{2}\right)$. By the choice of $L=\log ^{3}(n)$ and $\eta=n^{-0.08}$, we have $\mathcal{T}_{\text {mat }}(n, n, k)=n^{2} \log ^{2}(n)$ and hence the cost is $O\left(n^{2} \log ^{3}(n)\right)=O\left(n^{2} L\right)$. 
Summary For the cost of the rest of the algorithm, we note that step 1,2 and 3 simply involves rearrangements and it takes $O(n T)$ for all of them, much faster than $O\left(n^{2} T\right)$. Finally, we note that Theorem 6.3 requires having an approximate explicit matrix inverse. This can be done by matrix maintenance (Theorem B.4) which takes $n^{2} L$ per phase. To see this, we note that Theorem B.4 takes $O\left(n^{2}\right)$ time per rank 1 update and it takes $O\left(n^{2}\right)$ time per unit multiplicative $\ell_{2}$ changes in the weight (if we pick $\epsilon=0.01$ in Theorem B.4). Hence, each phase, the cost is just $O\left(n^{2} L\right)$.

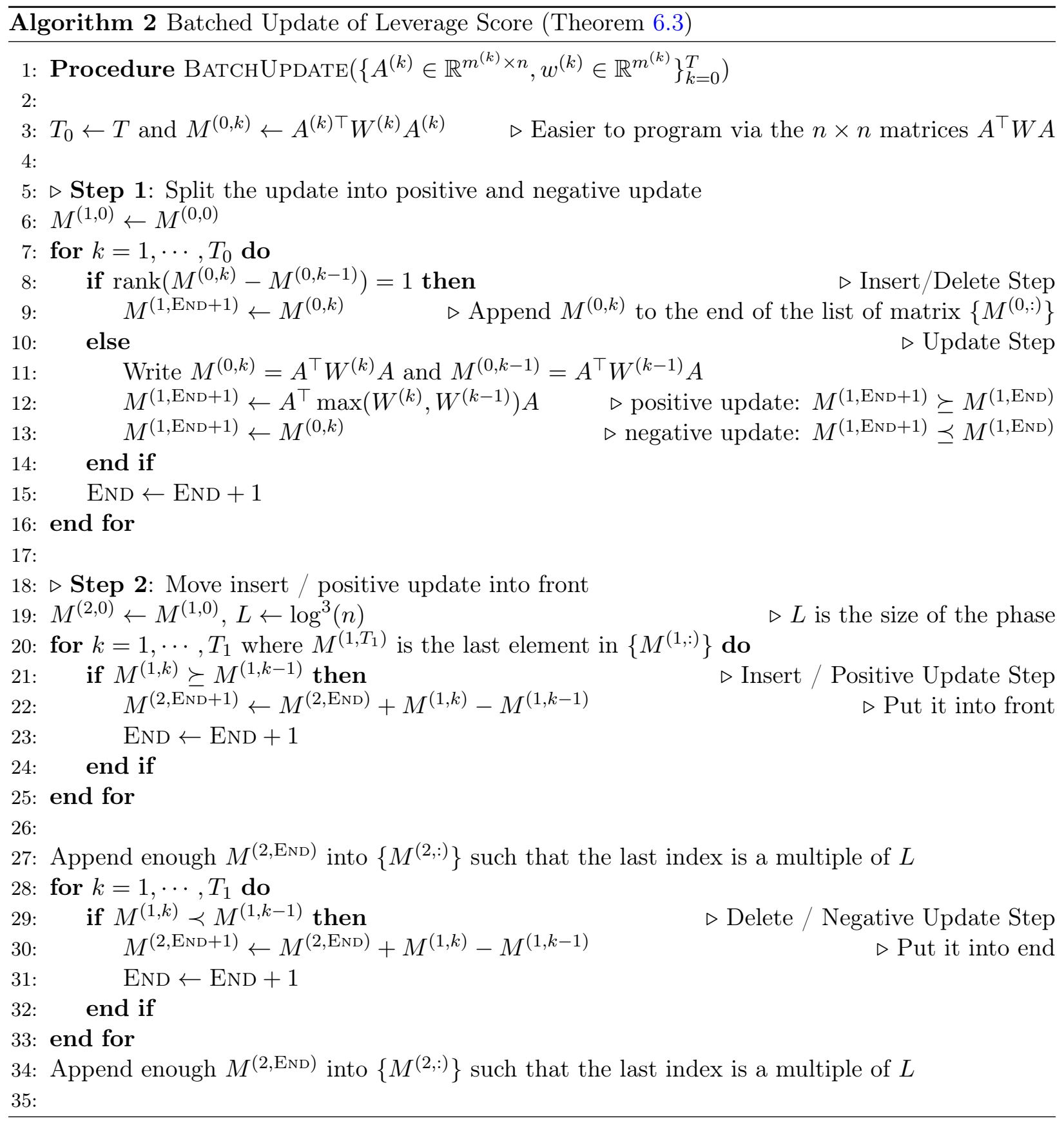




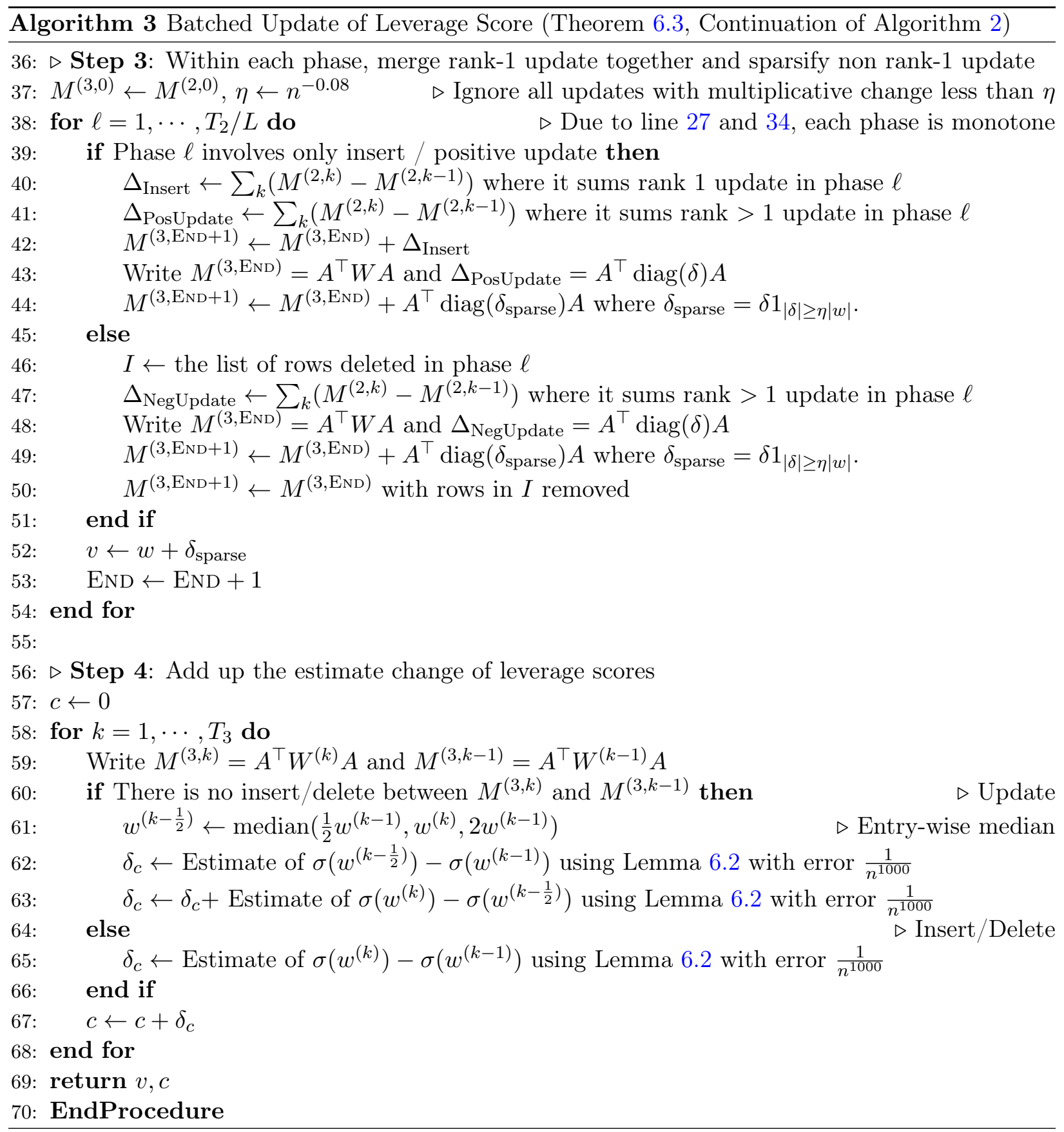




\section{Simple Deterministic Leverage Score Maintenance}

In this section, we give a simple deterministic leverage score maintenance data-structure which is used by both the inner and middle phases in our main data structure in Section 5. As a subprocedure, we make use of the batched low-rank update procedure in Section 6 which requires the following assumption for a sequence of updates acts:

Assumption 7.1 (Short sequence and small updates). Let the sequence of matrices and vectors in acts be $A^{(0)}, A^{(1)}, \cdots, A^{(T)} \in \mathbb{R}^{m \times n}$ and $w^{(0)}, w^{(1)}, \cdots, w^{(T)} \in \mathbb{R}^{m}$. We assume that $T \leq n^{0.08}$, the maximum number of rows is bounded by $O(n)$, and that the changes satisfy

- Update: $\left\|\log w^{(k)}-\log w^{(k-1)}\right\|_{2} \leq 0.01$.

- Insert/Delete: $w_{a} a a^{\top} \preceq 0.01\left(A^{(k-1)}\right)^{\top} W^{(k-1)} A^{(k-1)}$.

\subsection{Main result}

Theorem 7.2 (Simple leverage score maintenance). Given an initial matrix $A \in \mathbb{R}^{m \times n}$ with $m=$ $O(n)$, initial weight $w \in \mathbb{R}_{+}^{m}$, and error parameter $\epsilon_{\text {simp }} \leq 0.01$. There is a deterministic data structure (Algorithm 4) that approximately maintains the leverage scores

$$
\sigma_{i}(w)=\left(\sqrt{W} A\left(A^{\top} W A\right)^{-1} A^{\top} \sqrt{W}\right)_{i, i},
$$

for positive diagonal matrices $W=\operatorname{diag}(w)$ through the following operations:

1. $\operatorname{Init}\left(A, w, \epsilon_{\text {simp }}\right)$ : takes $O\left(n^{\omega+o(1)}\right)$ time to initialize the data structure.

2. UPDATE(acts): update the matrix A, vector $w$ and the approximation of the leverage scores for the sequence of updates in acts. Moreover, if acts satisfies Assumption \%.1, then the function UPDATE(acts) makes one call to PM.UPDATE ${ }^{2}$ and takes extra time $O\left(n^{2} \cdot \mid\right.$ acts $\left.\mid\right)$ to compute a vector $\Delta \widetilde{\sigma} \in \mathbb{R}^{m}$ such that

$$
\|\Delta \widetilde{\sigma}-\Delta \sigma\|_{2} \leq \frac{1}{n^{100}}+O\left(\epsilon_{\operatorname{simp}}+\mid \text { acts } \mid \cdot n^{-0.08}\right) \cdot \mid \text { acts } \mid .
$$

3. $\operatorname{RefineEstimate}\left(\widetilde{\sigma}^{\text {new }}\right)$ : takes $O(n)$ time to update the approximation of $\sigma(w)$ to $\widetilde{\sigma}^{\text {new }}$.

4. QUERY(): takes $O(n)$ time to output the approximation $\widetilde{\sigma} \in \mathbb{R}^{m}$ maintained by the data structure.

Proof of Theorem 7.2. We only need to prove the guarantee for the function UPDATE(acts), as the guarantees for other functions are straightforward. Notice that each call to the function UPDATE(acts) makes at most one call to PM.UPDATE, and the extra running time of $O\left(n^{2} \cdot \mid\right.$ acts $\left.\mid\right)$ follows directly from Theorem 6.3. To prove the error guarantee, we let the sequence of matrices and vectors in acts be $A^{(0)}, A^{(1)}, \cdots, A^{(T)}$ and $w^{(0)}, w^{(1)}, \cdots, w^{(T)}$. notice that by Theorem 6.3 , we have

$$
\left\|\sigma_{A^{(T)}}\left(w^{\text {mid }}\right)-\sigma_{A^{(0)}}\left(w^{(0)}\right)-\Delta \widetilde{\sigma}^{\text {mid }}\right\|_{2} \leq \frac{1}{n^{100}} .
$$

It follows from Lemma 7.6 that

$$
\left\|\sigma_{A^{(T)}}\left(w^{(T)}\right)-\sigma_{A^{(T)}}\left(w^{\text {mid }}\right)-\Delta \tilde{\sigma}^{\text {new }}\right\|_{2} \leq O\left(\epsilon_{\text {simp }}+\mid \text { acts } \mid \cdot n^{-0.08}\right) \cdot \mid \text { acts } \mid .
$$

The error guarantee then follows by summing up the two error upper bounds above.

\footnotetext{
${ }^{2}$ The running time of procedure UPDATE depends on $\epsilon_{\text {simp }}$, see details in Theorem B.1
} 
The following corollary is an immediate consequence of Theorem 7.2 and B.1. It states that if matrix multiplication can be performed fast enough, then the simple leverage score maintenance would imply a deterministic $O\left(n^{3} \log (n / \epsilon)\right)$ algorithm for the cutting plane method.

Corollary 7.3. If $\mathcal{T}_{\text {mat }}(n, n, r)=O\left(n^{2} \log O(1)(n)\right)$ for $r=n^{\alpha}$ with $\alpha>2 / 3$, then there's a deterministic $O\left(n^{3} \log (n / \epsilon)\right)$ time algorithm for the cutting plane method.

Proof. This algorithm only uses the inner and middle phases. The inner phase is run as is our main algorithm. For the middle phase, we notice that, in this case, the exponent of matrix multiplication time can be bounded as $\omega<3-2 / 3=7 / 3$. Therefore, the data structure is restarted after every $n^{1 / 3}$ calls to the middle phase. For the middle phase, we use the simple leverage score maintenance data structure in Theorem 7.2 with parameter $\epsilon_{\text {simp }}=n^{-\alpha / 2} / \log ^{O(1)}(n)$. It follows from Theorem 7.2 that the error accumulated in the $n^{0.2}$ steps is $O\left(n^{-\alpha / 2} n^{1 / 3} \log O(1)(n)\right)=n^{-\Omega(1)}$. By Theorem 7.2 and 5.1, the running time is $O\left(n^{2} \log ^{O(1)}(n)\right)$ per step for middle phase which is fast enough. This finishes the proof of the corollary.

\subsection{Approximate leverage score's moving}

Lemma 7.4 (Leverage score's moving). Given any matrix $A \in \mathbb{R}^{m \times n}$, vectors $w^{\text {mid }}, w^{\text {new }} \in \mathbb{R}_{+}^{m}$. The change in leverage score from $w^{\text {mid }}$ to $w^{\text {new }}$ can be written as

$$
\begin{aligned}
\Delta \sigma_{i}^{\text {new }} & :=\sigma\left(w^{\text {new }}\right)_{i}-\sigma\left(w^{\text {mid }}\right)_{i} \\
& =\left(w_{i}^{\text {new }}-w_{i}^{\text {mid }}\right) \cdot \tau\left(w^{\text {mid }}\right)_{i}+w_{i}^{\text {new }} \cdot \int_{0}^{1} e_{i}^{\top} Q\left(x_{t}\right)\left(W^{\text {mid }}-W^{\text {new }}\right) Q\left(x_{t}\right) e_{i} \mathrm{~d} t
\end{aligned}
$$

where we define $x_{t}=w^{\text {mid }}+t\left(w^{\text {new }}-w^{\text {mid }}\right)$ for each $t \in[0,1]$.

Proof. We can rewrite $\sigma\left(w^{\text {new }}\right)_{i}-\sigma\left(w^{\text {mid }}\right)_{i}$ as

$$
\sigma\left(w^{\text {new }}\right)_{i}-\sigma\left(w^{\text {mid }}\right)_{i}=\left(w_{i}^{\text {new }}-w_{i}^{\text {mid }}\right) \cdot \tau\left(w^{\text {mid }}\right)_{i}+w_{i}^{\text {new }}\left(\tau\left(w^{\text {new }}\right)_{i}-\tau\left(w^{\text {mid }}\right)_{i}\right) .
$$

Then the second term is computed as

$$
\begin{aligned}
& w_{i}^{\text {new }}\left(\tau\left(w^{\text {new }}\right)_{i}-\tau\left(w^{\text {mid }}\right)_{i}\right) \\
= & w_{i}^{\text {new }} \cdot\left(\left(A\left(A^{\top} W^{\text {new }} A\right)^{-1} A^{\top}\right)_{i, i}-\left(A\left(A^{\top} W^{\text {mid }} A\right)^{-1} A^{\top}\right)_{i, i}\right) \\
= & w_{i}^{\text {new }} \cdot \int_{0}^{1} e_{i}^{\top} Q\left(x_{t}\right)\left(W^{\text {mid }}-W^{\text {new }}\right) Q\left(x_{t}\right) e_{i} \mathrm{~d} t .
\end{aligned}
$$

where the last step follows by the definition of $Q(v)=A\left(A^{\top} V A\right)^{-1} A^{\top} \in \mathbb{R}^{m \times m}$.

Lemma 7.5 (Computing $\Delta \widetilde{\sigma}^{\text {new }}$ ). Given any matrix $A \in \mathbb{R}^{m \times n}$, vectors $w^{\text {mid }}, w^{\text {new }} \in \mathbb{R}_{+}^{m}$, where $m=O(n)$. Define an approximation $\Delta \widetilde{\sigma}_{i}^{\text {new }}$ to the change in leverage score to be

$$
\Delta \widetilde{\sigma}_{i}^{\text {new }}=\left(w_{i}^{\text {new }}-w_{i}^{\text {mid }}\right) \cdot e_{i}^{\top} Q\left(v^{\text {mid }}\right) e_{i}+w_{i}^{\text {new }} e_{i}^{\top} Q\left(v^{\text {mid }}\right)\left(W^{\text {mid }}-W^{\text {new }}\right) Q\left(v^{\text {mid }}\right) e_{i} .
$$

Then given the matrix $Q\left(v^{\text {mid }}\right)$, we can compute $\Delta \widetilde{\sigma}_{i}^{\text {new }}$ for all $i \in[m]$ in time $O\left(n^{2}\right)$. 


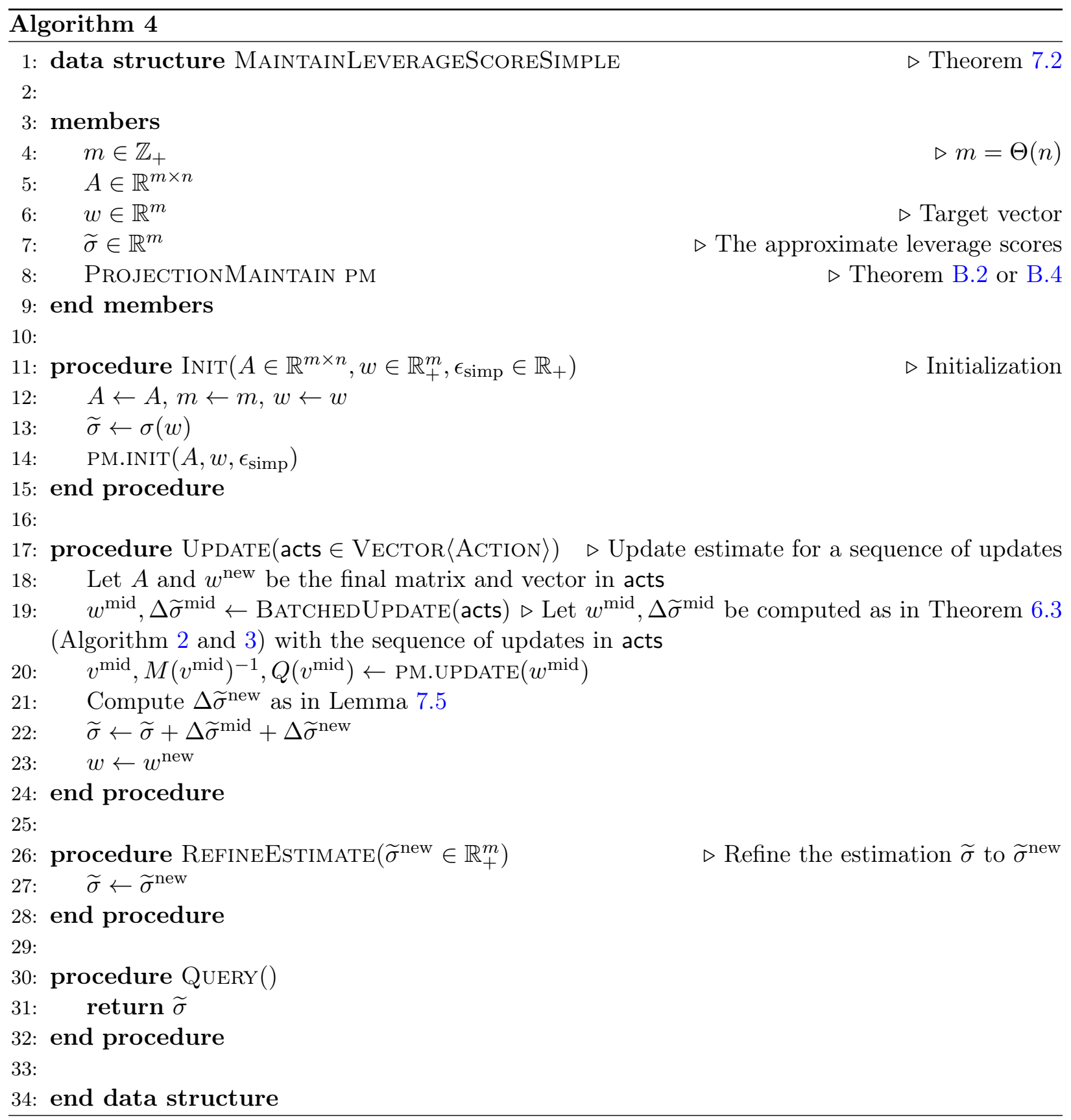


Proof. Since we know the matrix $Q\left(v^{\text {mid }}\right)$, the first term is essentially reading all the diagonal entries of matrix $Q\left(v^{\mathrm{mid}}\right)$, which can be computed for all $i \in[m]$ in time $O(n)$. For the second term, we denote $Q=Q\left(x_{t}\right)$ and $Q^{(2)}$ the entry-wise square of $Q$. Then we have

$$
\begin{aligned}
& w_{i}^{\text {new }} e_{i}^{\top} Q\left(W^{\text {mid }}-W^{\text {new }}\right) Q e_{i} \\
= & w_{i}^{\text {new }} \cdot \sum_{j \in[m]}\left(Q_{i, j}\right)^{2}\left(w^{\text {mid }}-w^{\text {new }}\right)_{j} \\
= & w_{i}^{\text {new }} \cdot e_{i}^{\top} Q^{(2)}\left(w^{\text {mid }}-w^{\text {new }}\right) .
\end{aligned}
$$

Therefore, in order to compute the second term for all $i \in[m]$, it suffices to compute the matrixvector product $Q^{(2)}\left(w^{\text {mid }}-w^{\text {new }}\right)$, which takes time $O\left(n^{2}\right)$.

\section{$7.3 \quad \ell_{2}$-error bound}

Let $A^{(T)}$ and $A^{(0)}$ be the final and initial matrix in the sequence of updates in acts, and $w^{(0)}$ be the initial vector in acts. Denote $\Delta \sigma^{\text {mid }}=\sigma_{A^{(T)}}\left(w^{\text {mid }}\right)-\sigma_{A^{(0)}}\left(w^{(0)}\right)$. It follows from Theorem 6.3 that if Assumption 7.1 holds, then we have

$$
\left\|\Delta \widetilde{\sigma}^{\text {mid }}-\Delta \sigma^{\text {mid }}\right\|_{2} \leq \frac{1}{n^{100}}
$$

It therefore suffices to bound the $\ell_{2}$-error $\left\|\Delta \widetilde{\sigma}^{\text {new }}-\Delta \sigma^{\text {new }}\right\|_{2}$. For simplicity, we use $A$ to denote $A^{(T)}$. Recall from Lemma 7.4 that

$$
\begin{aligned}
\Delta \sigma_{i}^{\text {new }} & :=\sigma\left(w^{\text {new }}\right)_{i}-\sigma\left(w^{\text {mid }}\right)_{i} \\
& =\left(w_{i}^{\text {new }}-w_{i}^{\text {mid }}\right) \cdot \tau\left(w^{\text {mid }}\right)_{i}+w_{i}^{\text {new }} \cdot \int_{0}^{1} e_{i}^{\top} Q\left(x_{t}\right)\left(W^{\text {mid }}-W^{\text {new }}\right) Q\left(x_{t}\right) e_{i} \mathrm{~d} t
\end{aligned}
$$

where $x_{t}=w^{\text {mid }}+t\left(w^{\text {new }}-w^{\text {mid }}\right)$ for each $t \in[0,1]$. Also recall that in Lemma 7.5 , our approximation $\Delta \widetilde{\sigma}^{\text {new }}$ is computed as

$$
\Delta \widetilde{\sigma}_{i}^{\text {new }}=\left(w_{i}^{\text {new }}-w_{i}^{\text {mid }}\right) \cdot e_{i}^{\top} Q\left(v^{\text {mid }}\right) e_{i}+w_{i}^{\text {new }} e_{i}^{\top} Q\left(v^{\text {mid }}\right)\left(W^{\text {mid }}-W^{\text {new }}\right) Q\left(v^{\text {mid }}\right) e_{i} .
$$

We use $\Delta_{1}$ and $\Delta_{2}$ to denote the two corresponding error terms

$$
\begin{aligned}
& \left(\Delta_{1}\right)_{i}=\left(w_{i}^{\text {new }}-w_{i}^{\text {mid }}\right) \cdot e_{i}^{\top} Q\left(v^{\text {mid }}\right) e_{i}-\left(w_{i}^{\text {new }}-w_{i}^{\text {mid }}\right) \cdot \tau\left(w^{\text {mid }}\right)_{i} \\
& \left(\Delta_{2}\right)_{i}=w_{i}^{\text {new }} e_{i}^{\top} Q\left(v^{\text {mid }}\right)\left(W^{\text {mid }}-W^{\text {new }}\right) Q\left(v^{\text {mid }}\right) e_{i}-w_{i}^{\text {new }} \cdot \int_{0}^{1} e_{i}^{\top} Q\left(x_{t}\right)\left(W^{\text {mid }}-W^{\text {new }}\right) Q\left(x_{t}\right) e_{i} \mathrm{~d} t
\end{aligned}
$$

where $x_{t}=w^{\text {mid }}+t\left(w^{\text {new }}-w^{\text {mid }}\right)$. It follows that

$$
\left\|\Delta \widetilde{\sigma}^{\text {new }}-\Delta \sigma^{\text {new }}\right\|_{2} \leq\left\|\Delta_{1}\right\|_{2}+\left\|\Delta_{2}\right\|_{2} .
$$

We prove the following bound on the $\ell_{2}$-error.

Lemma 7.6 ( $\ell_{2}$-error bound). Assume Assumption 7.1 holds and that $\epsilon_{\text {simp }} \leq 0.01$, where $\epsilon_{\text {simp }}$ is the error parameter in Algorithm 4. Then the $\ell_{2}$-error is upper bounded as

$$
\left\|\Delta \widetilde{\sigma}^{\text {new }}-\Delta \sigma^{\text {new }}\right\|_{2} \leq O\left(\epsilon_{\text {simp }}+\mid \text { acts } \mid \cdot n^{-0.08}\right) \cdot \mid \text { acts } \mid \text {. }
$$


Proof. For simplicity, we assume here that $w^{\text {new }}-w^{\text {mid }} \geq 0$ and that $w^{\text {mid }}-v^{\text {mid }} \geq 0$. This is without loss of generality, since in the case where these assumptions don't hold, we can break the corresponding terms into positive and negative parts and bound each one of them. The lemma then follows immeditely from the following Lemma 7.7 and 7.8 .

Lemma $7.7\left(\left\|\Delta_{1}\right\|_{2}\right)$. Assume Assumption 7.1 holds and that $\epsilon_{\mathrm{simp}} \leq 0.01$. Then we have the following upper bound on $\left\|\Delta_{1}\right\|_{2}$

$$
\left\|\Delta_{1}\right\|_{2} \leq O\left(\epsilon_{\mathrm{simp}}\right) \cdot \mid \text { acts } \mid
$$

Proof. Recall the definition of the error term $\Delta_{1}$ as

$$
\left(\Delta_{1}\right)_{i}=\left(w_{i}^{\text {new }}-w_{i}^{\text {mid }}\right) \cdot e_{i}^{\top} Q\left(v^{\text {mid }}\right) e_{i}-\left(w_{i}^{\text {new }}-w_{i}^{\text {mid }}\right) \cdot \tau\left(w^{\text {mid }}\right)_{i} .
$$

We define $y_{s}=w^{\text {mid }}+s\left(v^{\text {mid }}-w^{\text {mid }}\right)$ for $s \in[0,1]$. The term $\left(\Delta_{1}\right)_{i}$ can be rewritten as

$$
\begin{aligned}
\left(\Delta_{1}\right)_{i} & =\left(w_{i}^{\text {new }}-w_{i}^{\text {mid }}\right) \cdot e_{i}^{\top} Q\left(v^{\text {mid }}\right) e_{i}-\left(w_{i}^{\text {new }}-w_{i}^{\text {mid }}\right) \cdot \tau\left(w^{\text {mid }}\right)_{i} \\
& =\left(w_{i}^{\text {new }}-w_{i}^{\text {mid }}\right) \cdot e_{i}^{\top}\left(\int_{0}^{1} Q\left(y_{s}\right)\left(W^{\text {mid }}-V^{\text {mid }}\right) Q\left(y_{s}\right) \mathrm{d} s\right) e_{i} \\
& =e_{i}^{\top} \sqrt{W^{\text {new }}-W^{\text {mid }}} Q\left(y_{\xi}\right)\left(W^{\text {mid }}-V^{\text {mid }}\right) Q\left(y_{\xi}\right) \sqrt{W^{\text {new }}-W^{\text {mid }}} e_{i},
\end{aligned}
$$

for some $\xi \in[0,1]$. It follows that

$$
\begin{aligned}
\left\|\Delta_{1}\right\|_{2}^{2} & \leq\left\|\sqrt{\frac{W^{\text {new }}-W^{\text {mid }}}{Y_{\xi}}} P\left(y_{\xi}\right) \frac{W^{\text {mid }}-V^{\text {mid }}}{Y_{\xi}} P\left(y_{\xi}\right) \sqrt{\frac{W^{\text {new }}-W^{\text {mid }}}{Y_{\xi}}}\right\|_{F}^{2} \\
& =\operatorname{tr}\left[(\sqrt{C} P B P \sqrt{C})^{2}\right]
\end{aligned}
$$

where in the last step we define diagonal matrices $B, C \in \mathbb{R}^{m \times m}$ as

$$
B=\frac{W^{\mathrm{mid}}-V^{\mathrm{mid}}}{Y_{\xi}}, C=\frac{W^{\mathrm{new}}-W^{\mathrm{mid}}}{Y_{\xi}},
$$

and we use $P$ to denote $P\left(y_{\xi}\right)$. Then we have

$$
\begin{aligned}
& \operatorname{tr}\left[(\sqrt{C} P B P \sqrt{C})^{2}\right] \\
\leq & \|b\|_{\infty}^{2} \cdot \operatorname{tr}\left[C^{2}\right] \\
= & O(1) \cdot\left\|\log \left(w^{\text {mid }}\right)-\log \left(v^{\text {mid }}\right)\right\|_{\infty}^{2} \cdot\left\|\log \left(w^{\text {new }}\right)-\log \left(w^{\text {mid }}\right)\right\|_{2}^{2} \\
\leq & O\left(\epsilon_{\text {simp }}^{2}\right) \cdot\left\|\log \left(w^{\text {new }}\right)-\log \left(w^{\text {mid }}\right)\right\|_{2}^{2} \\
\leq & O\left(\epsilon_{\text {simp }}^{2}\right) \cdot \mid \text { acts }\left.\right|^{2}
\end{aligned}
$$

where the last step follows from Theorem 6.3.

Lemma $7.8\left(\left\|\Delta_{2}\right\|_{2}\right)$. Assume Assumption $\% .1$ holds and that $\epsilon_{\mathrm{simp}} \leq 0.01$. Then we have the following upper bound on $\left\|\Delta_{2}\right\|_{2}$

$$
\left\|\Delta_{2}\right\|_{2} \leq O\left(\epsilon_{\mathrm{simp}}+\mid \text { acts } \mid \cdot n^{-0.08}\right) \cdot \mid \text { acts } \mid .
$$


Proof. Recall the definition of the error term $\Delta_{2}$ as

$$
\left(\Delta_{2}\right)_{i}=w_{i}^{\text {new }} e_{i}^{\top} Q\left(v^{\text {mid }}\right)\left(W^{\text {mid }}-W^{\text {new }}\right) Q\left(v^{\text {mid }}\right) e_{i}-w_{i}^{\text {new }} \cdot \int_{0}^{1} e_{i}^{\top} Q\left(x_{t}\right)\left(W^{\text {mid }}-W^{\text {new }}\right) Q\left(x_{t}\right) e_{i} \mathrm{~d} t,
$$

where $x_{t}=w^{\text {mid }}+t\left(w^{\text {new }}-w^{\text {mid }}\right)$. It follows that there exists $t \in[0,1]$ such that

$$
\begin{aligned}
\left(\Delta_{2}\right)_{i} & =w_{i}^{\text {new }} e_{i}^{\top} Q\left(v^{\text {mid }}\right)\left(W^{\text {mid }}-W^{\text {new }}\right) Q\left(v^{\text {mid }}\right) e_{i}-w_{i}^{\text {new }} e_{i}^{\top} Q\left(x_{t}\right)\left(W^{\text {mid }}-W^{\text {new }}\right) Q\left(x_{t}\right) e_{i} \\
& =\int_{0}^{1} \frac{\mathrm{d}}{\mathrm{d} s}\left(e_{i}^{\top} \sqrt{W^{\text {new }}} Q\left(v_{t, s}\right)\left(W^{\text {mid }}-W^{\text {new }}\right) Q\left(v_{t, s}\right) \sqrt{W^{\text {new }}} e_{i}\right) \mathrm{d} s
\end{aligned}
$$

where in the last line we define $v_{t, s}=x_{t}+s\left(v^{\text {mid }}-x_{t}\right)$ for $s \in[0,1]$. Therefore, in order to bound the $\left\|\Delta_{2}\right\|_{i}$, it suffices to bound the $\ell_{2}$-norm of the derivative:

$$
\begin{aligned}
\left\|\Delta_{2}\right\|_{2} & \leq \int_{0}^{1}\left\|\left(\frac{\mathrm{d}}{\mathrm{d} s}\left(e_{i}^{\top} \sqrt{W^{\text {new }}} Q\left(v_{t, s}\right)\left(W^{\text {mid }}-W^{\text {new }}\right) Q\left(v_{t, s}\right) \sqrt{W^{\text {new }}} e_{i}\right)\right)_{i=1}^{m}\right\|_{2} \mathrm{~d} s \\
& \leq \sup _{s \in[0,1]}\left\|\left(\frac{\mathrm{d}}{\mathrm{d} s}\left(e_{i}^{\top} \sqrt{W^{\text {new }}} Q\left(v_{t, s}\right)\left(W^{\text {mid }}-W^{\text {new }}\right) Q\left(v_{t, s}\right) \sqrt{W^{\text {new }}} e_{i}\right)\right)_{i=1}^{m}\right\|_{2} .
\end{aligned}
$$

Notice that

$$
\begin{aligned}
& \frac{\mathrm{d}}{\mathrm{d} s}\left(e_{i}^{\top} \sqrt{W^{\text {new }}} Q\left(v_{t, s}\right)\left(W^{\text {mid }}-W^{\text {new }}\right) Q\left(v_{t, s}\right) \sqrt{W^{\text {new }}} e_{i}\right) \\
= & -2 e_{i}^{\top} \sqrt{W^{\text {new }}} Q\left(v_{t, s}\right)\left(V^{\text {mid }}-X_{t}\right) Q\left(v_{t, s}\right)\left(W^{\text {mid }}-W^{\text {new }}\right) Q\left(v_{t, s}\right) \sqrt{W^{\text {new }}} e_{i} .
\end{aligned}
$$

It follows that for any $s \in[0,1]$, we have

$$
\begin{aligned}
& \left\|\left(\frac{\mathrm{d}}{\mathrm{d} s}\left(e_{i}^{\top} \sqrt{W^{\text {new }}} Q\left(v_{t, s}\right)\left(W^{\text {mid }}-W^{\text {new }}\right) Q\left(v_{t, s}\right) \sqrt{W^{\text {new }}} e_{i}\right)\right)_{i=1}^{m}\right\|_{2}^{2} \\
\leq & 4 \cdot\left\|\sqrt{W^{\text {new }}} Q\left(v_{t, s}\right)\left(V^{\text {mid }}-X_{t}\right) Q\left(v_{t, s}\right)\left(W^{\text {mid }}-W^{\text {new }}\right) Q\left(v_{t, s}\right) \sqrt{W^{\text {new }}}\right\|_{F}^{2} \\
= & 4 \operatorname{tr}[\sqrt{D} P C P B P D P B P C P \sqrt{D}],
\end{aligned}
$$

where in the last step we define diagonal matrices $B, C \in \mathbb{R}^{m \times m}$ as

$$
B=\frac{W^{\text {mid }}-W^{\text {new }}}{V_{t, s}}, C=\frac{V^{\text {mid }}-X_{t}}{V_{t, s}}, D=\frac{W^{\text {new }}}{V_{t, s}},
$$

and we use $P$ to denote $P\left(v_{t, s}\right)$. By Assumption 7.1, we have

$$
\left\|\log \left(w^{\text {new }}\right)-\log \left(w^{\text {mid }}\right)\right\|_{\infty} \leq 0.01 .
$$

This together with the assumption that $\epsilon_{\text {simp }} \leq 0.01$ further implies that

$$
\left\|\log \left(w^{\text {new }}\right)-\log \left(v_{s, t}\right)\right\|_{\infty} \leq 0.1
$$


Therefore, we have

$$
\begin{array}{rlr} 
& \operatorname{tr}[\sqrt{D} P C P B P D P B P C P \sqrt{D}] \\
\leq & O(1) \cdot \operatorname{tr}\left[\sqrt{D} P C P B^{2} P C P \sqrt{D}\right] & P D P \preceq O(1) \cdot I \\
= & O(1) \cdot \operatorname{tr}[B P C P D P C P B] & \\
\leq & O(1) \cdot \operatorname{tr}\left[B P C^{2} P B\right] & P D P \preceq O(1) \cdot I \\
\leq & O(1) \cdot\|c\|_{\infty}^{2} \cdot\|b\|_{2}^{2} & P C^{2} P \preceq\|c\|_{\infty}^{2} \cdot I \\
\leq & O(1) \cdot\left\|\log \left(v^{\text {mid }}\right)-\log \left(x_{t}\right)\right\|_{\infty}^{2} \cdot\left\|\log \left(w^{\text {new }}\right)-\log (w)\right\|_{2}^{2} & \\
\leq & O(1) \cdot\left(\epsilon_{\text {simp }}+\left\|\log \left(w^{\text {new }}\right)-\log \left(w^{\text {mid }}\right)\right\|_{\infty}\right)^{2} \cdot\left\|\log \left(w^{\text {new }}\right)-\log (w)\right\|_{2}^{2} . &
\end{array}
$$

The lemma then follows from Theorem 6.3 which gives $\left\|\log \left(w^{\text {new }}\right)-\log \left(w^{\text {mid }}\right)\right\|_{\infty} \leq \mid$ acts $\mid \cdot n^{-0.08}$ and $\left\|\log \left(w^{\text {new }}\right)-\log \left(w^{\text {mid }}\right)\right\|_{2} \leq 0.01 \mid$ acts $\mid$. 


\section{Complicated Randomized Leverage Score Maintenance}

In this section, we present a complicated randomized leverage score maintenance data structure which is used by the outer phase in our main data structure in Section 5. We again make crucial use of the batched low-rank update procedure in Section 6.

\subsection{Main result}

Theorem 8.1 (Complicated leverage score maintenance). Given an initial matrix $A \in \mathbb{R}^{m \times n}$ with $m=O(n)$, initial weight $w \in \mathbb{R}_{+}^{m}$, JL dimension $r>0$, discrete sampling parameter $N \in \mathbb{Z}_{+}$, and error parameter $\epsilon_{\mathrm{comp}}<0.01$. There is a randomized data structure (Algorithm 5) that approximately maintains the leverage scores

$$
\sigma_{i}(w)=\left(\sqrt{W} A\left(A^{\top} W A\right)^{-1} A^{\top} \sqrt{W}\right)_{i, i}
$$

for positive diagonal matrices $W=\operatorname{diag}(w)$ through the following operations:

1. $\operatorname{Init}\left(A, w, r, N, \epsilon_{\mathrm{comp}}\right)$ : takes $O\left(n^{\omega+o(1)}\right)$ time to initialize the data structure.

2. Update(acts): update the matrix A, vector $w$ and the approximation of the leverage scores for the sequence of updates in acts. Moreover, if acts satisfies Assumption 7.1, then the function UPDATE(acts) makes $O(N)$ calls to PM.UPDATE and takes extra time $O\left(n^{2} \cdot \mid\right.$ acts $\mid+$ $\left.\mathcal{T}_{\text {mat }}(n, n, r) \cdot N^{3} \cdot \log (n)\right)$ to compute a random vector $\Delta \widetilde{\sigma} \in \mathbb{R}^{m}$ with error upper bounded by

$$
\|\Delta \sigma-\mathbf{E}[\Delta \widetilde{\sigma}]\|_{2} \leq \frac{1}{n^{100}}+O\left(\operatorname{poly}(n) / 2^{2 N}\right),
$$

and variance upper bounded by

$$
\mathbf{E}\left[\left\|\sigma\left(w^{\text {new }}\right)-\sigma(w)-\Delta \widetilde{\sigma}\right\|_{2}^{2}\right] \leq O\left(N^{3} / r\right) \cdot \epsilon_{\text {comp }}^{2} \cdot \mid \text { acts }\left.\right|^{2} .
$$

3. RefineEstimate $\left(\widetilde{\sigma}^{\text {new }}\right)$ : takes $O(n)$ time to update the approximation of $\sigma(w)$ to $\widetilde{\sigma}^{\text {new }}$.

4. QUeRY () : takes $O(n)$ time to output the approximation $\widetilde{\sigma} \in \mathbb{R}^{m}$ maintained by the data structure.

Proof of Theorem 8.1. We only need to prove the guarantee for the function UPDATE(acts), as the guarantees for other functions are straightforward. The running time upper bound for the function $\operatorname{UpDATE}\left(\right.$ acts) is given in Lemma 8.7. The upper bound on the $\ell_{2}$-error $\|\Delta \sigma-\mathbf{E}[\Delta \widetilde{\sigma}]\|_{2}$ follows from Theorem 6.3 and the lemmas in Section 8.6. The upper bound on the variance follows from the lemmas in Section 8.4 and 8.5.

\subsection{Leverage score's moving}

Definition $8.2\left(x_{t}, z_{t}\right.$ and $\left.y_{s, t}\right)$. Given vectors $w^{\text {mid }}, w^{\text {new }} \in \mathbb{R}_{+}^{m}$ and $v^{\text {mid }} \in \mathbb{R}_{+}^{m}$. For all $t \in[0,1]$, we define $x_{t}$ to be $w^{\text {mid }}+t\left(w^{\text {new }}-w^{\text {mid }}\right)$.

To define $z_{t}$ for $t \in[0,1]$, we first define $z_{t}$ for all $t \in \mathcal{T}$ as in Algorithm 5. We then extend the definition of $z_{t}$ to the entire interval $[0,1]$ by connecting consecutive points with segments.

For any $s \in[0,1]$, we define $y_{s, t}=z_{t}+s\left(x_{t}-z_{t}\right)$. 


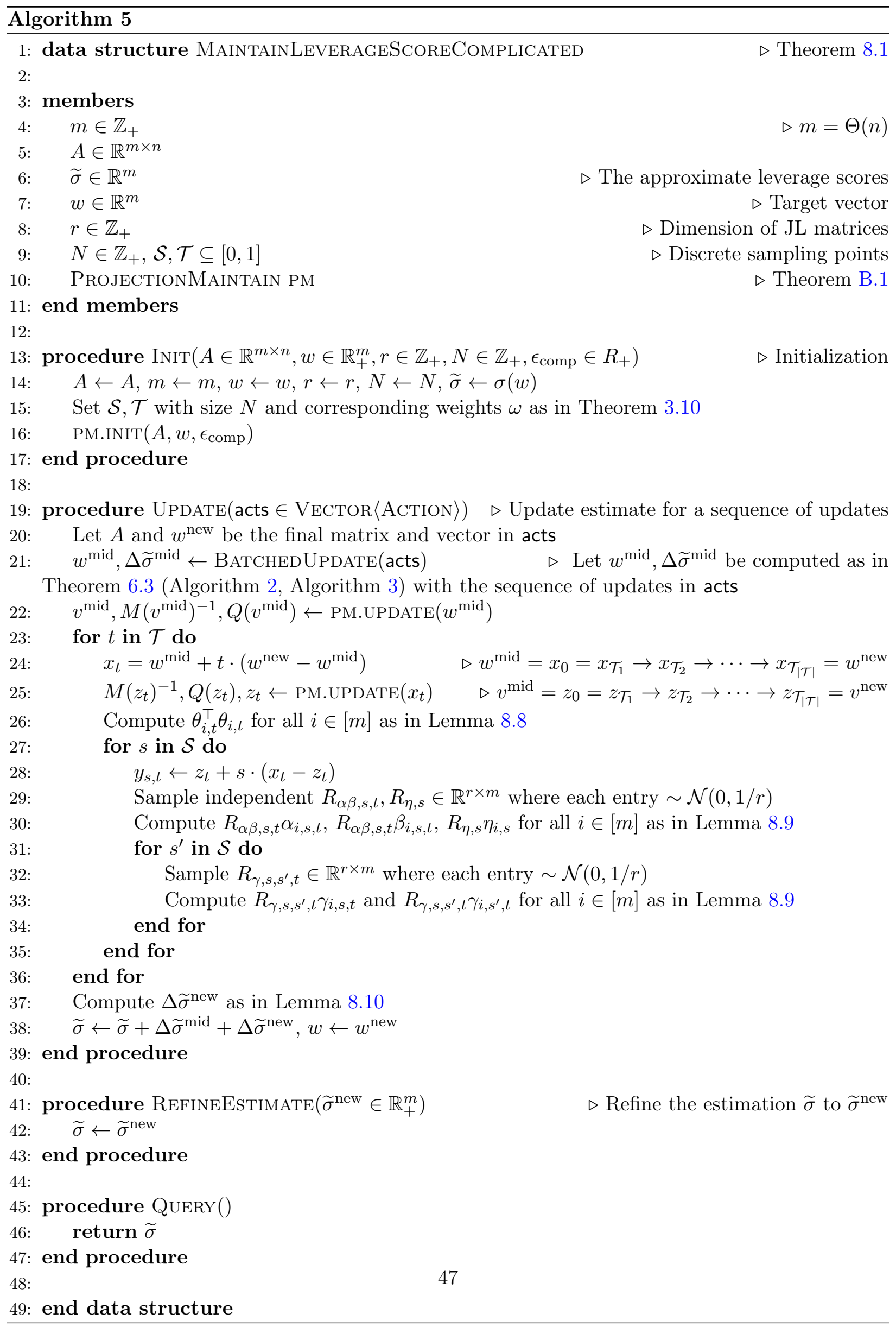




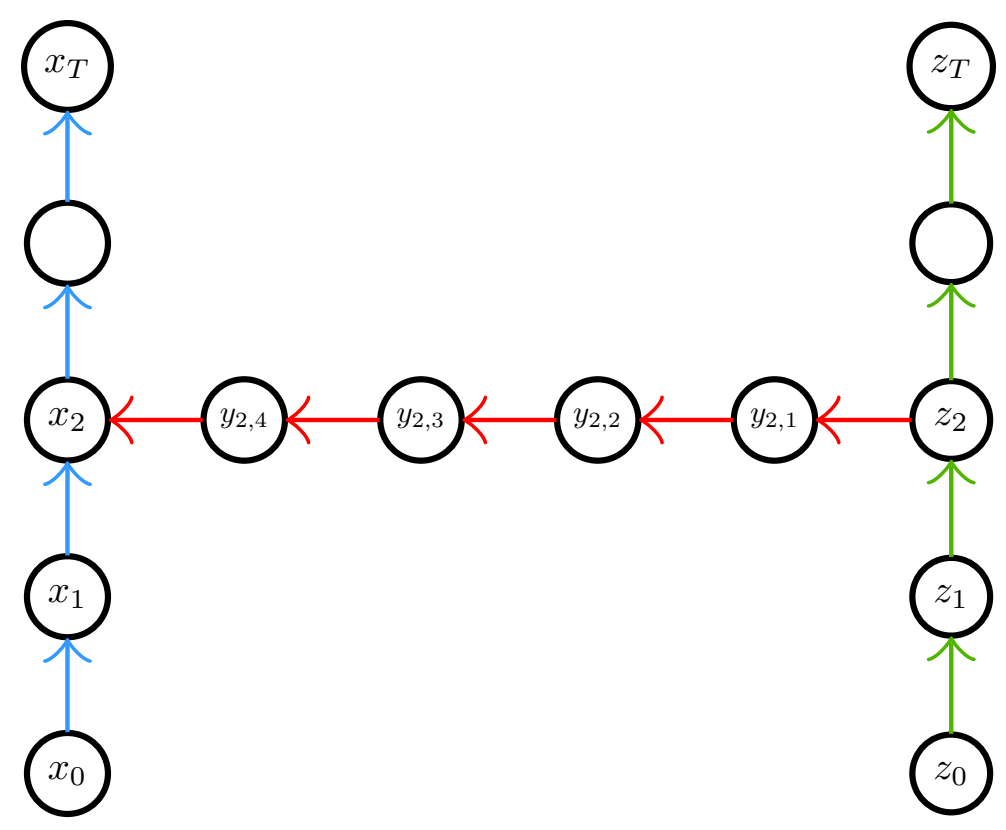

Figure 2: This figure is related to Algorithm 5.

Definition $8.3(\eta, \theta, \alpha, \beta$ and $\gamma)$. We define

$$
\begin{aligned}
\eta_{i, s} & =\sqrt{Z_{0}-X_{0}} \cdot Q\left(y_{s, 0}\right) \cdot \sqrt{W^{\text {mid }}-W^{\text {new }}} \cdot e_{i} \\
\theta_{i, t} & =\sqrt{W^{\text {mid }}-W^{\text {new }}} \cdot Q\left(z_{t}\right) \cdot \sqrt{W^{\text {new }}} \cdot e_{i}, \\
\alpha_{i, s, t} & =\sqrt{Z_{t}-X_{t}} \cdot Q\left(y_{s, t}\right) \cdot \sqrt{W^{\text {new }}} \cdot e_{i}, \\
\beta_{i, s, t} & =\sqrt{Z_{t}-X_{t}} \cdot Q\left(y_{s, t}\right) \cdot\left(W^{\text {mid }}-W^{\text {new }}\right) \cdot Q\left(z_{t}\right) \cdot \sqrt{W^{\text {new }}} \cdot e_{i}, \\
\gamma_{i, s, t} & =\sqrt{W^{\text {mid }}-W^{\text {new }}} \cdot Q\left(y_{s, t}\right) \cdot\left(Z_{t}-X_{t}\right) \cdot Q\left(y_{s, t}\right) \cdot \sqrt{W^{\text {new }}} \cdot e_{i} .
\end{aligned}
$$

Here we make the simplifying assumption that $\left(Z_{t}-X_{t}\right)$ is PSD for every $t \in[0,1]$ and that $\left(W^{\text {mid }}-W^{\text {new }}\right)$ is PSD. In the case where these might not hold, we can simply break these matrices into positive and negative parts and bound both parts using essentially the same calculations.

Lemma 8.4 (Leverage score's moving). The change in leverage score can be written as

$$
\begin{aligned}
\sigma\left(w^{\text {new }}\right)_{i}-\sigma\left(w^{\text {mid }}\right)_{i}= & \int_{0}^{1} \theta_{i, t}^{\top} \theta_{i, t} \mathrm{~d} t+2 \int_{0}^{1} \int_{0}^{1} \alpha_{i, s, t}^{\top} \beta_{i, s, t} \mathrm{~d} s \mathrm{~d} t+\int_{0}^{1} \int_{0}^{1} \int_{0}^{1} \gamma_{i, s, t}^{\top} \gamma_{i, s^{\prime}, t} \mathrm{~d} s \mathrm{~d} s^{\prime} \mathrm{d} t \\
& +\tau\left(v^{\text {mid }}\right)_{i} \cdot\left(w_{i}^{\text {new }}-w_{i}^{\text {mid }}\right)-\int_{0}^{1} \eta_{i, s}^{\top} \eta_{i, s} \mathrm{~d} s .
\end{aligned}
$$

Proof. We first rewrite $\sigma\left(w^{\text {new }}\right)_{i}-\sigma\left(w^{\text {mid }}\right)_{i}$ into two terms

$$
\begin{aligned}
\sigma\left(w^{\text {new }}\right)_{i}-\sigma\left(w^{\text {mid }}\right)_{i} & =w_{i}^{\text {new }} \tau\left(w^{\text {new }}\right)_{i}-w_{i}^{\text {mid }} \tau\left(w^{\text {mid }}\right)_{i} \\
& =w_{i}^{\text {new }} \cdot\left(\tau\left(w^{\text {new }}\right)_{i}-\tau\left(w^{\text {mid }}\right)_{i}\right)+\tau\left(w^{\text {mid }}\right)_{i} \cdot\left(w_{i}^{\text {new }}-w_{i}^{\text {mid }}\right) .
\end{aligned}
$$

The lemma then follows immediately from the following Lemma 8.5 and 8.6.

Lemma 8.5 (First term in leverage score's moving). For the first term in Lemma 8.4, we have $w_{i}^{\text {new }} \cdot\left(\tau\left(w^{\text {new }}\right)_{i}-\tau\left(w^{\text {mid }}\right)_{i}\right)=\int_{0}^{1} \theta_{i, t}^{\top} \theta_{i, t} \mathrm{~d} t+2 \int_{0}^{1} \int_{0}^{1} \alpha_{i, s, t}^{\top} \beta_{i, s, t} \mathrm{~d} s \mathrm{~d} t+\int_{0}^{1} \int_{0}^{1} \int_{0}^{1} \gamma_{i, s, t}^{\top} \gamma_{i, s^{\prime}, t} \mathrm{~d} s \mathrm{~d} s^{\prime} \mathrm{d} t$. 
Proof. We can compute $\tau\left(w^{\text {new }}\right)_{i}-\tau\left(w^{\text {mid }}\right)_{i}$ as

$$
\begin{aligned}
& \tau\left(w^{\text {new }}\right)_{i}-\tau\left(w^{\text {mid }}\right)_{i} \\
= & \left(A\left(A^{\top} W^{\text {new }} A\right)^{-1} A^{\top}\right)_{i, i}-\left(A\left(A^{\top} W^{\text {mid }} A\right)^{-1} A^{\top}\right)_{i, i} \\
= & {\left[\int_{0}^{1} A\left(A^{\top}\left(W^{\text {mid }}+t\left(W^{\text {new }}-W^{\text {mid }}\right)\right) A\right)^{-1} A^{\top}\left(W^{\text {mid }}-W^{\text {new }}\right) A\left(A^{\top}\left(W^{\text {mid }}+t\left(W^{\text {new }}-W^{\text {mid }}\right)\right) A\right)^{-1} A^{\top} \mathrm{d} t\right]_{i, i} } \\
= & {\left[\int_{0}^{1} Q\left(x_{t}\right)\left(W^{\text {mid }}-W^{\text {new }}\right) Q\left(x_{t}\right) \mathrm{d} t\right]_{i, i}, }
\end{aligned}
$$

where the last step follows by the definition of $Q(v)=A\left(A^{\top} V A\right)^{-1} A^{\top} \in \mathbb{R}^{m \times m}$ and $x_{t}=w^{\text {mid }}+$ $t\left(w^{\text {new }}-w^{\text {mid }}\right)$. Now we write $Q\left(x_{t}\right)-Q\left(z_{t}\right)$ in a similar way as follows:

$$
\begin{aligned}
Q\left(x_{t}\right)-Q\left(z_{t}\right) & =A\left(A^{\top} X_{t} A\right)^{-1} A^{\top}-A\left(A^{\top} Z_{t} A\right)^{-1} A^{\top} \\
& =\int_{0}^{1} Q\left(y_{s, t}\right) \cdot\left(Z_{t}-X_{t}\right) \cdot Q\left(y_{s, t}\right) \mathrm{d} s,
\end{aligned}
$$

where recall the definition of $y_{s, t}=z_{t}+s\left(x_{t}-z_{t}\right)$. Using the above expressions, we can rewrite $w_{i}^{\text {new }} \cdot\left(\tau\left(w^{\text {new }}\right)_{i}-\tau\left(w^{\text {mid }}\right)_{i}\right)$ as follows:

$$
\begin{aligned}
& w_{i}^{\text {new }} \cdot\left(\tau\left(w^{\text {new }}\right)_{i}-\tau\left(w^{\text {mid }}\right)_{i}\right) \\
= & w_{i}^{\text {new }} \cdot\left[\int_{0}^{1}\left(Q\left(z_{t}\right)+\int_{0}^{1} Q\left(y_{s, t}\right) \cdot\left(Z_{t}-X_{t}\right) \cdot Q\left(y_{s, t}\right) \mathrm{d} s\right) \cdot\left(W^{\text {mid }}-W^{\text {new }}\right)\right. \\
& \left.\cdot\left(Q\left(z_{t}\right)+\int_{0}^{1} Q\left(y_{s^{\prime}, t}\right) \cdot\left(Z_{t}-X_{t}\right) \cdot Q\left(y_{s^{\prime}, t}\right) \mathrm{d} s^{\prime}\right) \mathrm{d} t\right]_{i, i} \\
= & \int_{0}^{1} e_{i}^{\top} \sqrt{W^{\text {new }}} \cdot Q\left(z_{t}\right) \cdot\left(W^{\text {mid }}-W^{\text {new }}\right) \cdot Q\left(z_{t}\right) \cdot \sqrt{W^{\text {new }}} e_{i} \mathrm{~d} t \\
& +2 \int_{0}^{1} e_{i}^{\top} \sqrt{W^{\text {new }}}\left(\int_{0}^{1} Q\left(y_{s, t}\right) \cdot\left(Z_{t}-X_{t}\right) \cdot Q\left(y_{s, t}\right) \mathrm{d} s\right)\left(W^{\text {mid }}-W^{\text {new }}\right) Q\left(z_{t}\right) \sqrt{W^{\text {new }}} e_{i} \mathrm{~d} t \\
& +\int_{0}^{1} e_{i}^{\top} \sqrt{W^{\text {new }}}\left(\int_{0}^{1} Q\left(y_{s, t}\right) \cdot\left(Z_{t}-X_{t}\right) \cdot Q\left(y_{s, t}\right) \mathrm{d} s\right) \cdot\left(W^{\text {mid }}-W^{\text {new }}\right) \\
& \cdot\left(\int_{0}^{1} Q\left(y_{s^{\prime}, t}\right) \cdot\left(Z_{t}-X_{t}\right) \cdot Q\left(y_{s^{\prime}, t}\right) \mathrm{d} s^{\prime}\right) \sqrt{W^{\text {new }}} e_{i} \mathrm{~d} t \\
= & \int_{0}^{1} \theta_{i, t}^{\top} \theta_{i, t} \mathrm{~d} t+2 \int_{0}^{1} \int_{0}^{1} \alpha_{i, s, t}^{\top} \beta_{i, s, t} \mathrm{~d} s \mathrm{~d} t+\int_{0}^{1} \int_{0}^{1} \int_{0}^{1} \gamma_{i, s, t}^{\top} \gamma_{i, s^{\prime}, t} \mathrm{~d} s \mathrm{~d} s^{\prime} \mathrm{d} t .
\end{aligned}
$$

Lemma 8.6 (Second term in leverage score's moving). For the second term in Lemma 8.4, we have

$$
\tau\left(w^{\text {mid }}\right)_{i} \cdot\left(w_{i}^{\text {new }}-w_{i}^{\text {mid }}\right)=\tau\left(v^{\text {mid }}\right)_{i} \cdot\left(w_{i}^{\text {new }}-w_{i}^{\text {mid }}\right)-\int_{0}^{1} \eta_{i, s}^{\top} \eta_{i, s} \mathrm{~d} s .
$$

Proof. We can rewrite $\tau\left(w^{\text {mid }}\right)_{i} \cdot\left(w_{i}^{\text {new }}-w_{i}^{\text {mid }}\right)$ as

$$
\tau\left(w^{\text {mid }}\right)_{i} \cdot\left(w_{i}^{\text {new }}-w_{i}^{\text {mid }}\right)=\tau\left(v^{\text {mid }}\right)_{i} \cdot\left(w_{i}^{\text {new }}-w_{i}^{\text {mid }}\right)+\left(\tau\left(w^{\text {mid }}\right)_{i}-\tau\left(v^{\text {mid }}\right)_{i}\right) \cdot\left(w_{i}^{\text {new }}-w_{i}^{\text {mid }}\right) .
$$


The second term can be computed as

$$
\begin{aligned}
& \left(w_{i}^{\text {new }}-w_{i}^{\text {mid }}\right) \cdot\left(\tau\left(w^{\text {mid }}\right)_{i}-\tau\left(v^{\text {mid }}\right)_{i}\right) \\
= & \left(w_{i}^{\text {new }}-w_{i}^{\text {mid }}\right) \cdot\left(\tau\left(x_{0}\right)_{i}-\tau\left(z_{0}\right)_{i}\right) \\
= & \left(w_{i}^{\text {new }}-w_{i}^{\text {mid }}\right) \cdot\left[\int_{0}^{1} Q\left(z_{0}+s\left(x_{0}-z_{0}\right)\right) \cdot\left(Z_{0}-X_{0}\right) \cdot Q\left(z_{0}+s\left(x_{0}-z_{0}\right)\right) \mathrm{d} s\right]_{i, i} \\
= & -\int_{0}^{1} e_{i}^{\top} \cdot \sqrt{W^{\text {mid }}-W^{\text {new }}} \cdot Q\left(y_{s, 0}\right) \cdot\left(Z_{0}-X_{0}\right) \cdot Q\left(y_{s, 0}\right) \cdot \sqrt{W^{\text {mid }}-W^{\text {new }}} \cdot e_{i} \mathrm{~d} s \\
= & -\int_{0}^{1} \eta_{i, s}^{\top} \eta_{i, s} \mathrm{~d} s .
\end{aligned}
$$

\subsection{Running time anlaysis}

Lemma 8.7 (Update time). Assume the sequence acts satisfies Assumption $\% .1$ and $\epsilon_{\mathrm{comp}} \leq 0.01$. Then each call to the function UPDATE(acts) makes $O(N)$ calls to PM.UPDATE and takes an extra $O\left(n^{2} \cdot \mid\right.$ acts $\left.\mid+\mathcal{T}_{\text {mat }}(n, n, r) \cdot N^{3} \cdot \log (n)\right)$ time.

Proof. The number of calls to PM.UpDATE directly follows from Algorithm 5. The time to compute $\Delta \widetilde{\sigma}^{\text {mid }}$ is $O\left(n^{2} \cdot \mid\right.$ acts $\left.\mid\right)$ by applying Theorem 6.3. The remaining part of the running time is given by the following Lemma 8.8, 8.9 and 8.10.

Lemma 8.8 (Computing $\theta^{\top} \theta$ ). Assume $m=O(n)$. Then for any $t \in \mathcal{T}$, the time to compute $\theta_{i, t}^{\top} \theta_{i, t}$ for all $i \in[m]$ is $O\left(n^{2}\right)$.

Proof. Recall from Definition 8.3 that

$$
\theta_{i, t}=\sqrt{W^{\text {mid }}-W^{\text {new }}} \cdot Q\left(z_{t}\right) \cdot \sqrt{W^{\text {new }}} \cdot e_{i} .
$$

Denote $Q=Q\left(z_{t}\right)$ and $Q^{(2)}$ the entry-wise square of $Q$. We have

$$
\begin{aligned}
\theta_{i, t}^{\top} \theta_{i, t} & =w_{i}^{\text {new }} e_{i}^{\top} Q\left(W^{\text {mid }}-W^{\text {new }}\right) Q e_{i} \\
& =w_{i}^{\text {new }} \cdot \sum_{j \in[m]}\left(Q_{i, j}\right)^{2}\left(w^{\text {mid }}-w^{\text {new }}\right)_{j} \\
& =w_{i}^{\text {new }} \cdot e_{i}^{\top} Q^{(2)}\left(w^{\text {mid }}-w^{\text {new }}\right) .
\end{aligned}
$$

Therefore, in order to compute $\theta_{i, t}^{\top} \theta_{i, t}$ for all $i \in[m]$, it suffices to compute the matrix-vector product $Q^{(2)}\left(w^{\text {mid }}-w^{\text {new }}\right)$, which takes time $O\left(n^{2}\right)$.

Lemma 8.9 (Computing $\alpha, \beta, \gamma, \eta)$. Assume $m=O(n)$ and $\epsilon_{\text {comp }} \leq 0.01$. For any $r \geq 0, t \in \mathcal{T}$, $s, s^{\prime} \in \mathcal{S}$ and matrices $R_{\alpha \beta, s, t}, R_{\gamma, s, s^{\prime}, t}, R_{\eta, s} \in \mathbb{R}^{r \times m}$, the time to compute $R_{\alpha \beta, s, t} \alpha_{i, s, t}, R_{\alpha \beta, s, t} \beta_{i, s, t}$, $R_{\gamma, s, s^{\prime}, t} \gamma_{i, s, t}, R_{\gamma, s, s^{\prime}, t} \gamma_{i, s^{\prime}, t}, R_{\eta, s} \eta_{i, s}$ for all $i \in[m]$ (up to negligible error) is $O\left(\mathcal{T}_{\text {mat }}(n, n, r) \cdot \log (n)\right.$ ) using fast matrix multiplication.

Proof. We only describe how to compute $R_{\alpha \beta, s, t} \alpha_{i, s, t}$ for all $i \in[m]$ in time $O\left(\mathcal{T}_{\text {mat }}(n, n, r) \cdot \log (n)\right)$ as the rest of the calculations are similar. Notice that that computing $R_{\alpha \beta, s, t} \alpha_{i, s, t}$ for all $i \in[m]$ is essentially computing the matrix

$$
\left\{R_{\alpha \beta, s, t} \alpha_{i, s, t}\right\}_{i \in[m]}=R_{\alpha \beta, s, t} \sqrt{Z_{t}-X_{t}} \cdot A \cdot M\left(y_{s, t}\right)^{-1} \cdot A^{\top} \cdot \sqrt{W^{\text {new }}} .
$$


Assume we know the matrix $M\left(y_{s, t}\right)^{-1}$, then we can perform the computation from left to right, and it follows that each matrix multiplication here can be done in time $O\left(\mathcal{T}_{\text {mat }}(n, n, r)\right)$. To remove the assumption that we know $M\left(y_{s, t}\right)^{-1}$, we pre-condition on the matrix $M\left(z_{t}\right)^{-1}$ and apply Lemma 6.1 to compute $R_{\alpha \beta, s, t} \alpha_{i, s, t}$ for all $i \in[m]$ (up to negligible error) in time $O\left(\mathcal{T}_{\text {mat }}(n, n, r) \cdot \log (n)\right.$ ).

Lemma 8.10 (Computing $\Delta \widetilde{\sigma}^{\text {new }}$ ). Assume $m=O(n)$ and $\epsilon_{\mathrm{comp}} \leq 0.01$. Define an approximation $\Delta \widetilde{\sigma}_{i}^{\text {new }}$ to the change in leverage score to be

$$
\begin{aligned}
\Delta \widetilde{\sigma}_{i}^{\text {new }}=\tau\left(v^{\text {mid }}\right)_{i} \cdot\left(w_{i}^{\text {new }}-w_{i}^{\text {mid }}\right)+\sum_{t \in \mathcal{T}} \omega_{t} \theta_{i, t}^{\top} \theta_{i, t}-\sum_{s \in \mathcal{S}} \omega_{s} \eta_{i, s}^{\top} R_{\eta, s}^{\top} R_{\eta, s} \eta_{i, s} \\
\quad+2 \sum_{t \in \mathcal{T}} \sum_{s \in \mathcal{S}} \omega_{t} \omega_{s} \alpha_{i, s, t}^{\top} R_{\alpha \beta, s, t}^{\top} R_{\alpha \beta, s, t} \beta_{i, s, t}+\sum_{t \in \mathcal{T}} \sum_{s \in \mathcal{S}} \sum_{s^{\prime} \in \mathcal{S}} \omega_{t} \omega_{s} \omega_{s^{\prime}} \gamma_{i, s, t}^{\top} R_{\gamma, s, s^{\prime}, t}^{\top} R_{\gamma, s, s^{\prime}, t} \gamma_{i, s^{\prime}, t} .
\end{aligned}
$$

Then the time to compute $\Delta \widetilde{\sigma}_{i}^{\text {new }}$ for all $i \in[m]$ (up to negligible error) is $O\left(\mathcal{T}_{\text {mat }}(n, n, r) \cdot N^{3} \cdot \log (n)\right)$.

Proof. Since we know $\tau\left(v^{\text {mid }}\right)_{i}=Q\left(v^{\text {mid }}\right)_{i, i}$ and $w_{i}^{\text {new }}-w_{i}^{\text {mid }}$, we can compute the first term for all $i \in[m]$ in $O(n)$ time. By Lemma 8.8, the time to compute the second term for all $i \in[m]$ is $O\left(n^{2} \cdot N\right)$. It follows from Lemma 8.9 that the time to compute the rest of the terms for all $i \in[m]$ is $O\left(\mathcal{T}_{\text {mat }}(n, n, r) \cdot N^{3} \cdot \log (n)\right)$.

\subsection{Upper bounding $\eta, \alpha, \beta$ and $\gamma$}

From the computation in Section 8.5, the variance of the three terms where we applied JL matrices are bounded by $\sum_{i}\left\|\eta_{i}\right\|_{2}^{4}, \sum_{i}\left\|\alpha_{i}\right\|_{2}^{2}\left\|\beta_{i}\right\|_{2}^{2}$ and $\sum_{i}\left\|\gamma_{i}\right\|_{2}^{4}$. Our bounds for these terms are summarized in Table 6.

\begin{tabular}{|l|l|l|}
\hline Quantity & Bound & Lemma \\
\hline$\sum_{i=1}^{m}\left\|\eta_{i}\right\|_{2}^{4}$ & $\epsilon_{\text {comp }}^{2} \cdot\left\|\log \left(w^{\text {new }}\right)-\log \left(w^{\text {mid }}\right)\right\|_{2}^{2}$ & Lemma 8.11 \\
\hline$\sum_{i=1}^{m}\left\|\alpha_{i}\right\|_{2}^{2}\left\|\beta_{i}\right\|_{2}^{2}$ & $\epsilon_{\text {comp }}^{2} \cdot\left\|\log \left(w^{\text {new }}\right)-\log \left(w^{\text {mid }}\right)\right\|_{2}^{2}$ & Lemma 8.12 \\
\hline$\sum_{i=1}^{m}\left\|\gamma_{i}\right\|_{2}^{4}$ & $\epsilon_{\text {comp }}^{4} \cdot\left\|\log \left(w^{\text {new }}\right)-\log \left(w^{\text {mid }}\right)\right\|_{2}^{2}$ & Lemma 8.13 \\
\hline
\end{tabular}

Table 6: Upper bounds for $\sum_{i}\left\|\eta_{i}\right\|_{2}^{4}, \sum_{i}\left\|\alpha_{i}\right\|_{2}^{2}\left\|\beta_{i}\right\|_{2}^{2}$ and $\sum_{i}\left\|\gamma_{i}\right\|_{2}^{4}$, where $\eta, \alpha, \beta$ and $\gamma$ are defined in Definition 8.3.

\subsubsection{Upper bounding $\sum_{i=1}^{m}\left\|\eta_{i}\right\|_{2}^{4}$}

Lemma 8.11. Assume $\epsilon_{\mathrm{comp}} \leq 0.01$, where $\epsilon_{\mathrm{comp}}$ is the error parameter in Algorithm 5. Then for any $s \in \mathcal{S}$, we have

$$
\sum_{i=1}^{m}\left\|\eta_{i, s}\right\|_{2}^{4}=O\left(\epsilon_{\text {comp }}^{2}\right) \cdot\left\|\log \left(w^{\text {new }}\right)-\log \left(w^{\text {mid }}\right)\right\|_{2}^{2} .
$$

Proof. We recall the definition of vector $\eta_{i, s} \in \mathbb{R}^{m}$ from Definition 8.3 as follows:

$$
\eta_{i, s}=\sqrt{Z_{0}-X_{0}} \cdot Q\left(y_{s, 0}\right) \cdot \sqrt{W^{\text {mid }}-W^{\text {new }}} \cdot e_{i},
$$


where $y_{s, t}=z_{t}+s\left(x_{t}-z_{t}\right)$ and $Y_{s, t}=Z_{t}+s\left(X_{t}-Z_{t}\right)$. Recall from Definition 3.2 that $P(v)=$ $V^{1 / 2} A\left(A^{\top} V A\right)^{-1} A^{\top} V^{1 / 2}=V^{1 / 2} \cdot Q(v) \cdot V^{1 / 2}$. We can rewrite $\eta_{i, s}$ as

$$
\eta_{i, s}=\frac{\sqrt{Z_{0}-X_{0}}}{\sqrt{Y_{s, 0}}} P\left(y_{s, 0}\right) \frac{\sqrt{W^{\text {mid }}-W^{\text {new }}}}{\sqrt{Y_{s, 0}}} e_{i} .
$$

Therefore, we have

$$
\begin{aligned}
& \sum_{i=1}^{m}\left\|\eta_{i, s}\right\|_{2}^{4} \\
= & \sum_{i=1}^{m}\left(e_{i}^{\top} \frac{\sqrt{W^{\text {mid }}-W^{\text {new }}}}{\sqrt{Y_{s, 0}}} P\left(y_{s, 0}\right) \frac{\sqrt{Z_{0}-X_{0}}}{\sqrt{Y_{s, 0}}} \cdot \frac{\sqrt{Z_{0}-X_{0}}}{\sqrt{Y_{s, 0}}} P\left(y_{s, 0}\right) \frac{\sqrt{W^{\text {mid }}-W^{\text {new }}}}{\sqrt{Y_{s, 0}}} e_{i}\right)^{2} \\
\leq & \left\|\frac{\sqrt{W^{\text {mid }}-W^{\text {new }}}}{\sqrt{Y_{s, 0}}} P\left(y_{s, 0}\right) \frac{Z_{0}-X_{0}}{Y_{s, 0}} P\left(y_{s, 0}\right) \frac{\sqrt{W^{\text {mid }}-W^{\text {new }}}}{\sqrt{Y_{s, 0}}}\right\|_{F}^{2} \\
= & \operatorname{tr}\left[\frac{\sqrt{W^{\text {mid }}-W^{\text {new }}}}{\sqrt{Y_{s, 0}}} P\left(y_{s, 0}\right) \frac{Z_{0}-X_{0}}{Y_{s, 0}} P\left(y_{s, 0}\right) \frac{\sqrt{W^{\text {mid }}-W^{\text {new }}}}{\sqrt{Y_{s, 0}}}\right. \\
& \left.\cdot \frac{\sqrt{W^{\text {mid }}-W^{\text {new }}}}{\sqrt{Y_{s, 0}}} P\left(y_{s, 0}\right) \frac{Z_{0}-X_{0}}{Y_{s, 0}} P\left(y_{s, 0}\right) \frac{\sqrt{W^{\text {mid }}-W^{\text {new }}}}{\sqrt{Y_{s, 0}}}\right] \\
= & \operatorname{tr}\left[(\sqrt{B} P C P \sqrt{B})^{2}\right],
\end{aligned}
$$

where in the last step we define diagonal matrices $B, C \in \mathbb{R}^{m \times m}$ as

$$
B=\frac{W^{\text {mid }}-W^{\text {new }}}{Y_{s, 0}}, C=\frac{Z_{0}-X_{0}}{Y_{s, 0}},
$$

and for simplicity, we use $P$ to denote the projection matrix $P\left(y_{s, 0}\right)$. Since $\epsilon_{\text {comp }} \leq 0.01$, it follows that

$$
\left\|\log \left(y_{s, 0}\right)-\log \left(x_{0}\right)\right\|_{\infty} \leq 0.01
$$

It follows that

$$
\begin{aligned}
& \operatorname{tr}\left[(\sqrt{B} P C P \sqrt{B})^{2}\right] \\
& \leq\|c\|_{\infty}^{2} \cdot \operatorname{tr}\left[B^{2}\right] \quad P \cdot C \cdot P \preceq\|c\|_{\infty} \cdot I \\
& =\|c\|_{\infty}^{2} \cdot\|b\|_{2}^{2} \\
& =O(1) \cdot\left\|\log \left(z_{0}\right)-\log \left(x_{0}\right)\right\|_{\infty}^{2} \cdot\left\|\log \left(w^{\text {new }}\right)-\log \left(w^{\text {mid }}\right)\right\|_{2}^{2} \\
& =O\left(\epsilon_{\mathrm{comp}}^{2}\right) \cdot\left\|\log \left(w^{\mathrm{new}}\right)-\log \left(w^{\mathrm{mid}}\right)\right\|_{2}^{2} \text {. }
\end{aligned}
$$

\subsubsection{Upper bounding $\sum_{i=1}^{m}\left\|\alpha_{i}\right\|_{2}^{2}\left\|\beta_{i}\right\|_{2}^{2}$}

Lemma 8.12. Assume $\epsilon_{\mathrm{comp}} \leq 0.01$ and $\left\|\log \left(w^{\text {new }}\right)-\log \left(w^{\text {mid }}\right)\right\|_{\infty} \leq 0.01$. Then we have

$$
\sum_{i=1}^{m}\left\|\alpha_{i, s, t}\right\|_{2}^{2}\left\|\beta_{i, s, t}\right\|_{2}^{2}=O\left(\epsilon_{\text {comp }}^{2}\right) \cdot\left\|\log \left(w^{\text {new }}\right)-\log \left(w^{\text {mid }}\right)\right\|_{2}^{2} .
$$


Proof. We recall the definition of vector $\alpha_{i, s, t} \in \mathbb{R}^{m}$ and $\beta_{i, s, t} \in \mathbb{R}^{m}$ from Definition 8.3 as follows:

$$
\begin{aligned}
\alpha_{i, s, t} & =\sqrt{Z_{t}-X_{t}} \cdot Q\left(y_{s, t}\right) \cdot \sqrt{W^{\text {new }}} \cdot e_{i}, \\
\beta_{i, s, t} & =\sqrt{Z_{t}-X_{t}} \cdot Q\left(y_{s, t}\right) \cdot\left(W^{\text {mid }}-W^{\text {new }}\right) \cdot Q\left(z_{t}\right) \cdot \sqrt{W^{\text {new }}} \cdot e_{i},
\end{aligned}
$$

where recall that $y_{s, t}=z_{t}+s\left(x_{t}-z_{t}\right)$ and $Y_{s, t}=Z_{t}+s\left(X_{t}-Z_{t}\right)$. Recall from Definition 3.2 that $P(v)=V^{1 / 2} A\left(A^{\top} V A\right)^{-1} A^{\top} V^{1 / 2}=V^{1 / 2} \cdot Q(v) \cdot V^{1 / 2}$. We therefore can rewrite $\alpha_{i, s, t}$ and $\beta_{i, s, t}$ as

$$
\begin{aligned}
\alpha_{i, s, t} & =\frac{\sqrt{Z_{t}-X_{t}}}{\sqrt{Y_{s, t}}} P\left(y_{s, t}\right) \frac{\sqrt{W^{\text {new }}}}{\sqrt{Y_{s, t}}} e_{i}, \\
\beta_{i, s, t} & =\frac{\sqrt{Z_{t}-X_{t}}}{\sqrt{Y_{s, t}}} P\left(y_{s, t}\right) \frac{W^{\text {mid }}-W^{\text {new }}}{\sqrt{Z_{t} Y_{s, t}}} P\left(z_{t}\right) \frac{\sqrt{W^{\text {new }}}}{\sqrt{Z_{t}}} e_{i} .
\end{aligned}
$$

Our assumptions $\epsilon_{\text {comp }} \leq 0.01$ and $\left\|\log \left(w^{\text {new }}\right)-\log \left(w^{\text {mid }}\right)\right\|_{\infty} \leq 0.01$ imply that

$$
\left\|\log \left(y_{s, t}\right)-\log \left(w^{\text {new }}\right)\right\|_{\infty} \leq 0.1 \quad \text { and } \quad\left\|\log \left(z_{t}\right)-\log \left(w^{\text {new }}\right)\right\|_{\infty} \leq 0.1 .
$$

Therefore we can upper bound $\alpha_{i, s, t}$ as

$$
\begin{aligned}
\max _{i, s, t}\left\|\alpha_{i, s, t}\right\|_{2}^{2} & =\max _{i, s, t}\left\|\frac{\sqrt{Z_{t}-X_{t}}}{\sqrt{Y_{s, t}}} P\left(y_{s, t}\right) \frac{\sqrt{W^{\text {new }}}}{\sqrt{Y_{s, t}}} e_{i}\right\|_{2}^{2} \\
& \leq \max _{i, s, t}\left\|y_{s, t}^{-1}\left(z_{t}-x_{t}\right)\right\|_{\infty} \cdot\left\|\frac{\sqrt{W^{\text {new }}}}{\sqrt{Y_{s, t}}} e_{i}\right\|_{2}^{2} \\
& =O\left(\epsilon_{\mathrm{comp}}\right) .
\end{aligned}
$$

For $\beta_{i, s, t}$, we have

$$
\begin{aligned}
& \sum_{i=1}^{m}\left\|\beta_{i, s, t}\right\|_{2}^{2} \\
= & \sum_{i=1}^{m} e_{i}^{\top} \frac{\sqrt{W^{\text {new }}}}{\sqrt{Z_{t}}} P\left(z_{t}\right) \frac{W^{\text {mid }}-W^{\text {new }}}{\sqrt{Z_{t} Y_{s, t}}} P\left(y_{s, t}\right) \frac{Z_{t}-X_{t}}{Y_{s, t}} P\left(y_{s, t}\right) \frac{W^{\text {mid }}-W^{\text {new }}}{\sqrt{Z_{t} Y_{s, t}}} P\left(z_{t}\right) \frac{\sqrt{W^{\text {new }}}}{\sqrt{Z_{t}}} e_{i} \\
= & \operatorname{tr}\left[\frac{\sqrt{W^{\text {new }}}}{\sqrt{Z_{t}}} P\left(z_{t}\right) \frac{W^{\text {mid }}-W^{\text {new }}}{\sqrt{Z_{t} Y_{s, t}}} P\left(y_{s, t}\right) \frac{Z_{t}-X_{t}}{Y_{s, t}} P\left(y_{s, t}\right) \frac{W^{\text {mid }}-W^{\text {new }}}{\sqrt{Z_{t} Y_{s, t}}} P\left(z_{t}\right) \frac{\sqrt{W^{\text {new }}}}{\sqrt{Z_{t}}}\right] \\
= & \operatorname{tr}\left[\sqrt{D} P\left(z_{t}\right) B P\left(y_{s, t}\right) C P\left(y_{s, t}\right) B P\left(z_{t}\right) \sqrt{D}\right],
\end{aligned}
$$

where in the last step we define diagonal matrices

$$
B=\frac{W^{\text {mid }}-W^{\text {new }}}{\sqrt{Z_{t} Y_{s, t}}}, C=\frac{Z_{t}-X_{t}}{Y_{s, t}}, D=\frac{W^{\text {new }}}{Z_{t}} .
$$

Thus, we have

$$
\begin{aligned}
& \operatorname{tr}\left[\sqrt{D} P\left(z_{t}\right) B P\left(y_{s, t}\right) C P\left(y_{s, t}\right) B P\left(z_{t}\right) \sqrt{D}\right] \\
& \leq\|c\|_{\infty} \cdot \operatorname{tr}\left[\sqrt{D} P\left(z_{t}\right) B^{2} P\left(z_{t}\right) \sqrt{D}\right] \\
& P\left(y_{s, t}\right) \cdot C \cdot P\left(y_{s, t}\right) \preceq\|c\|_{\infty} \cdot I \\
& =\|c\|_{\infty} \cdot \operatorname{tr}\left[B P\left(z_{t}\right) D P\left(z_{t}\right) B\right] \\
& =O(1) \cdot\|c\|_{\infty} \cdot \operatorname{tr}\left[B^{2}\right] \\
& =O(1) \cdot\|c\|_{\infty} \cdot\|b\|_{2}^{2} \\
& =O\left(\epsilon_{\mathrm{comp}}\right) \cdot\left\|\log \left(w^{\text {new }}\right)-\log \left(w^{\text {mid }}\right)\right\|_{2}^{2} \text {. } \\
& P\left(z_{t}\right) \cdot D \cdot P\left(z_{t}\right) \preceq O(1) \cdot I
\end{aligned}
$$




\subsubsection{Upper bounding $\sum_{i=1}^{m}\left\|\gamma_{i}\right\|_{2}^{4}$}

Lemma 8.13. Assume $\epsilon_{\mathrm{comp}} \leq 0.01$ and $\left\|\log \left(w^{\text {new }}\right)-\log \left(w^{\mathrm{mid}}\right)\right\|_{\infty} \leq 0.01$. Then for any $s \in \mathcal{S}$ and $t \in \mathcal{T}$, we have

$$
\sum_{i=1}^{m}\left\|\gamma_{i, s, t}\right\|_{2}^{4}=O\left(\epsilon_{\text {comp }}^{4}\right) \cdot\left\|\log \left(w^{\text {new }}\right)-\log \left(w^{\text {mid }}\right)\right\|_{2}^{2} .
$$

Proof. We recall the definition of vector $\gamma_{i, s, t} \in \mathbb{R}^{m}$ from Definition 8.3 as follows:

$$
\gamma_{i, s, t}=\sqrt{W^{\text {mid }}-W^{\text {new }}} \cdot Q\left(y_{s, t}\right) \cdot\left(Z_{t}-X_{t}\right) \cdot Q\left(y_{s, t}\right) \cdot \sqrt{W^{\text {new }}} \cdot e_{i},
$$

where recall that $y_{s, t}=\left(z_{t}+s\left(x_{t}-z_{t}\right)\right)$ and $Y_{s, t}=\left(Z_{t}+s\left(X_{t}-Z_{t}\right)\right)$. Recall from Definition 3.2 that $P(v)=V^{1 / 2} A\left(A^{\top} V A\right)^{-1} A^{\top} V^{1 / 2}=V^{1 / 2} \cdot Q(v) \cdot V^{1 / 2}$. We therefore can rewrite $\gamma_{i, s, t}$ as

$$
\gamma_{i, s, t}=\frac{\sqrt{W^{\text {mid }}-W^{\text {new }}}}{\sqrt{Y_{s, t}}} \cdot P\left(y_{s, t}\right) \cdot \frac{Z_{t}-X_{t}}{Y_{s, t}} \cdot P\left(y_{s, t}\right) \cdot \frac{\sqrt{W^{\text {new }}}}{\sqrt{Y_{s, t}}} \cdot e_{i} .
$$

It follows that

$$
\begin{aligned}
& \sum_{i=1}^{m}\left\|\gamma_{i, s, t}\right\|_{2}^{4} \\
= & \sum_{i=1}^{m}\left(e_{i}^{\top} \frac{\sqrt{W^{\text {new }}}}{\sqrt{Y_{s, t}}} P\left(y_{s, t}\right) \frac{Z_{t}-X_{t}}{Y_{s, t}} P\left(y_{s, t}\right) \frac{W^{\text {mid }}-W^{\text {new }}}{Y_{s, t}} P\left(y_{s, t}\right) \frac{Z_{t}-X_{t}}{Y_{s, t}} P\left(y_{s, t}\right) \frac{\sqrt{W^{\text {new }}}}{\sqrt{Y_{s, t}}} e_{i}\right)^{2} \\
\leq & \left\|\frac{\sqrt{W^{\text {new }}}}{\sqrt{Y_{s, t}}} P\left(y_{s, t}\right) \frac{Z_{t}-X_{t}}{Y_{s, t}} P\left(y_{s, t}\right) \frac{W^{\text {mid }}-W^{\text {new }}}{Y_{s, t}} P\left(y_{s, t}\right) \frac{Z_{t}-X_{t}}{Y_{s, t}} P\left(y_{s, t}\right) \frac{\sqrt{W^{\text {new }}}}{\sqrt{Y_{s, t}}}\right\|_{F}^{2} \\
= & \operatorname{tr}\left[\frac{\sqrt{W^{\text {new }}}}{\sqrt{Y_{s, t}}} P\left(y_{s, t}\right) \frac{Z_{t}-X_{t}}{Y_{s, t}} P\left(y_{s, t}\right) \frac{W^{\text {mid }}-W^{\text {new }}}{Y_{s, t}} P\left(y_{s, t}\right) \frac{Z_{t}-X_{t}}{Y_{s, t}} P\left(y_{s, t}\right) \frac{\sqrt{W^{\text {new }}}}{\sqrt{Y_{s, t}}}\right. \\
= & \left.\frac{\sqrt{W^{\text {new }}}}{\sqrt{Y_{s, t}}} P\left(y_{s, t}\right) \frac{Z_{t}-X_{t}}{Y_{s, t}} P\left(y_{s, t}\right) \frac{W^{\text {mid }}-W^{\text {new }}}{Y_{s, t}} P\left(y_{s, t}\right) \frac{Z_{t}-X_{t}}{Y_{s, t}} P\left(y_{s, t}\right) \frac{\sqrt{W^{\text {new }}}}{\sqrt{Y_{s, t}}}\right] \\
= & \left.\operatorname{ri} P B P C P{ }^{2}\right] .
\end{aligned}
$$

where in the last step we define diagonal matrices $B, C, D \in \mathbb{R}^{m \times m}$,

$$
B=\frac{W^{\text {mid }}-W^{\text {new }}}{Y_{s, t}}, C=\frac{Z_{t}-X_{t}}{Y_{s, t}}, D=\frac{W^{\text {new }}}{Y_{s, t}} .
$$

and for simplicity, we use $P$ to denote $P\left(y_{s, t}\right)$. By our assumptions that $\epsilon_{\text {simp }} \leq 0.01$ and

$$
\left\|\log \left(w^{\text {new }}\right)-\log \left(w^{\text {mid }}\right)\right\|_{\infty} \leq 0.01,
$$

we have

$$
\left\|\log \left(y_{s, t}\right)-\log \left(w^{\text {new }}\right)\right\|_{\infty} \leq 0.1
$$


Therefore we have

$$
\begin{aligned}
& \operatorname{tr}\left[(\sqrt{D} P C P B P C P \sqrt{D})^{2}\right] \\
& =\operatorname{tr}\left[(\sqrt{B} P C P D P C P \sqrt{B})^{2}\right] \\
& \leq O(1) \cdot \operatorname{tr}\left[\left(\sqrt{B} P C^{2} P \sqrt{B}\right)^{2}\right] \quad P \cdot D \cdot P \preceq O(1) \cdot I \\
& \leq O(1) \cdot\|c\|_{\infty}^{4} \cdot \operatorname{tr}\left[B^{2}\right] \quad P \cdot C^{2} \cdot P \preceq\|c\|_{\infty}^{2} \cdot I \\
& =O(1) \cdot\|c\|_{\infty}^{4} \cdot\|b\|_{2}^{2} \\
& =O\left(\epsilon_{\mathrm{comp}}^{4}\right) \cdot\left\|\log \left(w^{\mathrm{new}}\right)-\log \left(w^{\mathrm{mid}}\right)\right\|_{2}^{2} .
\end{aligned}
$$

\subsection{Variance upper bound for random Gaussian matrices}

\begin{tabular}{|l|l|l|l|}
\hline Notation & Statement & Quantity & Upper bound \\
\hline$\eta^{\top} \eta$ & Lemma 8.15 & $\operatorname{Var}\left[\sum \eta^{\top} \eta-\sum \eta^{\top} R^{\top} R \eta\right]$ & $O(1 / r) \cdot \sum\left\|\eta_{i, s}\right\|_{2}^{4}$ \\
\hline$\alpha^{\top} \beta$ & Lemma 8.16 & $\operatorname{Var}\left[\sum \alpha^{\top} \beta-\sum \beta^{\top} R^{\top} R \alpha\right]$ & $O(1 / r) \cdot \sum\left\|\beta_{i, s, t}\right\|_{2}^{2}\left\|\alpha_{i, s, t}\right\|_{2}^{2}$ \\
\hline$\gamma^{\top} \gamma$ & Lemma 8.17 & $\operatorname{Var}\left[\sum \gamma^{\top} \gamma-\sum \gamma^{\top} R^{\mid} R \gamma\right]$ & $O(1 / r) \cdot \sum\left\|\gamma_{i, s, t}\right\|_{2}^{4}$ \\
\hline
\end{tabular}

Table 7: Variance upper bounds for $\eta^{\top} \eta, \alpha^{\top} \beta$ and $\gamma^{\top} \gamma$, where $\eta, \alpha, \beta$ and $\gamma$ are defined in Definition 8.3.

Our variance bounds for the terms where we applied JL matrices are summarized in Table 7. These results are consequences of the following Lemma 8.14.

\subsubsection{A variance bound for random Gaussian matrices}

Lemma 8.14 (JL variance bound). Given vectors $x, y \in \mathbb{R}^{m}$. Let $R \in \mathbb{R}^{r \times m}$ be a random Gaussian matrix with $R_{i, j} \sim \mathcal{N}(0,1 / r)$. Then we have

$$
\underset{R}{\mathbf{E}}\left[x^{\top} R^{\top} R y\right]=x^{\top} y, \quad \underset{R}{\operatorname{Var}}\left[x^{\top} R^{\top} R y\right] \leq \frac{3}{r} \cdot\|x\|_{2}^{2}\|y\|_{2}^{2} .
$$

Proof. Since $\mathbf{E}\left[R^{\top} R\right]=I$, we have

$$
\underset{R}{\mathbf{E}}\left[x^{\top} R^{\top} R y\right]=x^{\top} y
$$

Next we prove the bound on the variance. We use $R_{i}$ for each $i \in[r]$ to denote the column vector that corresponds to the $i$ th row of $R$. We have 


$$
\begin{aligned}
\underset{R}{\operatorname{Var}\left[x^{\top} R^{\top} R y\right]} & =\underset{R}{\operatorname{Var}}\left[\sum_{i \in[r]} x^{\top} R_{i} R_{i}^{\top} y\right] \\
& =r \underset{R_{1}}{\operatorname{Var}}\left[x^{\top} R_{1} R_{1}^{\top} y\right] \\
& \leq r \underset{R_{1}}{\mathbf{E}}\left[\left(x^{\top} R_{1} R_{1}^{\top} y\right)^{2}\right] \\
& \leq r\left(\underset{R_{1}}{\mathbf{E}}\left[\left(x^{\top} R_{1}\right)^{4}\right]\right)^{1 / 2} \cdot\left(\underset{R_{1}}{\mathbf{E}}\left[\left(y^{\top} R_{1}\right)^{4}\right]\right)^{1 / 2} \\
& \leq \frac{3}{r} \cdot\|x\|_{2}^{2}\|y\|_{2}^{2},
\end{aligned}
$$

where the last inequality is because $x^{\top} R_{1}$ is a Gaussian random variable with variance $\|x\|_{2}^{2} / r$.

\subsubsection{Variance bound for $\eta^{\top} \eta, \alpha^{\top} \beta$ and $\gamma^{\top} \gamma$}

The lemmas below all follow immediately from Lemma 8.14.

Lemma 8.15 (Variance bound for $\eta^{\top} \eta$ ). Let $\sigma_{i, \mathrm{dis}}$ and $\sigma_{i, \mathrm{jl}}$ be defined as follows

$$
\sigma_{i, \mathrm{dis}}=\sum_{s \in \mathcal{S}} \omega_{s} \eta_{i, s}^{\top} \eta_{i, s}, \quad \sigma_{i, \mathrm{jl}}=\sum_{s \in \mathcal{S}} \omega_{s} \eta_{i, s}^{\top} R_{\eta, s}^{\top} R_{\eta, s} \eta_{i, s}
$$

where $R_{\eta, s} \in \mathbb{R}^{r \times m}$ for $s \in \mathcal{S}$ are independent random Gaussian matrices with $\left(R_{\eta, s}\right)_{i, j} \sim \mathcal{N}(0,1 / r)$. Then, we have

$$
\underset{R}{\mathbf{E}}\left[\sigma_{i, \mathrm{jl}}\right]=\sigma_{i, \mathrm{dis}}, \quad \underset{R}{\operatorname{Var}}\left[\sigma_{i, \mathrm{jl}}\right] \leq \frac{3}{r} \cdot \sum_{s \in \mathcal{S}} \omega_{s}^{2}\left\|\eta_{i, s}\right\|_{2}^{4}
$$

Lemma 8.16 (Variance bound for $\alpha^{\top} \beta$ ). Let $\sigma_{i, \mathrm{dis}}$ and $\sigma_{i, \mathrm{jl}}$ be defined as follows

$$
\sigma_{i, \mathrm{dis}}=\sum_{t \in \mathcal{T}} \sum_{s \in \mathcal{S}} \omega_{t} \omega_{s} \beta_{i, s, t}^{\top} \alpha_{i, s, t}, \quad \sigma_{i, \mathrm{jl}}=\sum_{t \in \mathcal{T}} \sum_{s \in \mathcal{S}} \omega_{t} \omega_{s} \beta_{i, s, t}^{\top} R_{\alpha \beta, s, t}^{\top} R_{\alpha \beta, s, t} \alpha_{i, s, t} .
$$

where $R_{\alpha \beta, s, t} \in \mathbb{R}^{r \times m}$ for $s \in \mathcal{S}, t \in \mathcal{T}$ are independent random Gaussian matrices with $\left(R_{\alpha \beta, s, t}\right)_{i, j} \sim$ $\mathcal{N}(0,1 / r)$. Then, we have

$$
\underset{R}{\mathbf{E}}\left[\sigma_{i, \mathrm{j}}\right]=\sigma_{i, \mathrm{dis}}, \quad \underset{R}{\operatorname{Var}}\left[\sigma_{i, \mathrm{j}}\right] \leq \frac{3}{r} \sum_{t \in \mathcal{T}} \sum_{s \in \mathcal{S}} \omega_{t}^{2} \omega_{s}^{2}\left\|\beta_{i, s, t}\right\|_{2}^{2}\left\|\alpha_{i, s, t}\right\|_{2}^{2}
$$

Lemma 8.17 (Variance bound for $\gamma^{\top} \gamma$ ). Let $\sigma_{i, \mathrm{dis}}$ and $\sigma_{i, \mathrm{jl}}$ be defined as follows

$$
\sigma_{i, \mathrm{dis}}=\sum_{t \in \mathcal{T}} \sum_{s \in \mathcal{S}} \sum_{s^{\prime} \in \mathcal{S}} \omega_{t} \omega_{s} \omega_{s^{\prime}} \gamma_{i, s, t}^{\top} \gamma_{i, s^{\prime}, t}, \quad \sigma_{i, \mathrm{jl}}=\sum_{t \in \mathcal{T}} \sum_{s \in \mathcal{S}} \sum_{s^{\prime} \in \mathcal{S}} \omega_{t} \omega_{s} \omega_{s^{\prime}} \gamma_{i, s, t}^{\top} R_{\gamma, s, s^{\prime}, t}^{\top} R_{\gamma, s, s^{\prime}, t} \gamma_{i, s^{\prime}, t} .
$$

where $R_{\gamma, s, s^{\prime}, t} \in \mathbb{R}^{r \times m}$ are independent random Gaussian matrices with $\left(R_{\gamma, s, s^{\prime}, t}\right)_{i, j} \sim \mathcal{N}(0,1 / r)$. Then, we have

$$
\underset{R}{\mathbf{E}}\left[\sigma_{i, \mathrm{jl}}\right]=\sigma_{i, \mathrm{dis}}, \quad \underset{R}{\operatorname{Var}}\left[\sigma_{i, \mathrm{j}}\right] \leq \frac{3}{r} \sum_{t \in \mathcal{T}} \sum_{s \in \mathcal{S}} \sum_{s^{\prime} \in \mathcal{S}} \omega_{t}^{2} \omega_{s}^{2} \omega_{s^{\prime}}^{2}\left\|\gamma_{i, s, t}\right\|_{2}^{4}
$$




\begin{tabular}{|l|l|l|l|}
\hline Notation & Statement & Quantity & Upper bound \\
\hline$\eta^{\top} \eta$ & Lemma 8.18 & $\left|\int \eta^{\top} \eta-\sum \eta^{\top} \eta\right|$ & $O\left(\operatorname{poly}(n) / 2^{2 N}\right)$ \\
\hline$\theta^{\top} \theta$ & Lemma 8.20 & $\left|\int \theta^{\top} \theta-\sum \theta^{\top} \theta\right|$ & $O\left(\operatorname{poly}(n) / 2^{2 N}\right)$ \\
\hline$\alpha^{\top} \beta$ & Lemma 8.21 & $\left|\int \alpha^{\top} \beta-\sum \alpha^{\top} \beta\right|$ & $O\left(\operatorname{poly}(n) / 2^{2 N}\right)$ \\
\hline$\gamma^{\top} \gamma$ & Lemma 8.22 & $\left|\int \gamma^{\top} \gamma-\sum \gamma^{\top} \gamma\right|$ & $O\left(\operatorname{poly}(n) / 2^{2 N}\right)$ \\
\hline
\end{tabular}

Table 8: Error upper bounds for discrete sampling. Here, $\eta, \alpha, \beta$ and $\gamma$ are defined as in Definition 8.3.

\subsection{Error upper bound for discrete sampling}

Our error upper bounds for approximating integral by discrete sampling are summarized in Table 8. We only prove Lemma 8.18 in the following. The rest of the lemmas follows from similar arguments.

Lemma 8.18 (Error upper bound for $\eta^{\top} \eta$ ). Assume $\epsilon_{\text {comp }} \leq 0.01$. Let $\sigma_{i \text {,cts }}$ and $\sigma_{i, \text { dis }}$ be defined as follows

$$
\sigma_{i, \mathrm{cts}}=\int_{0}^{1} \eta_{i, s}^{\top} \eta_{i, s} \mathrm{~d} s, \quad \sigma_{i, \mathrm{dis}}=\sum_{s \in \mathcal{S}} \omega_{s} \eta_{i, s}^{\top} \eta_{i, s},
$$

where $|\mathcal{S}|=N$. Then we have

$$
\left|\sigma_{i, \mathrm{dis}}-\sigma_{i, \mathrm{cts}}\right| \leq O\left(\operatorname{poly}(n) / 2^{2 N}\right) .
$$

Proof. Define function $f:[0,1] \rightarrow \mathbb{R}$ to be $f(s)=\eta_{i, s}^{\top} \eta_{i, s}$. Applying Theorem 3.9 with $N=|\mathcal{S}|$, the error is bounded as

$$
\left|\sigma_{i, \mathrm{dis}}-\sigma_{i, \mathrm{cts}}\right| \leq \frac{M_{2 N}}{(2 N) ! \cdot 2^{2 N}}
$$

where

$$
M_{2 N}=\max _{s \in[0,1]} \frac{\partial^{(2 N)} f}{\partial s^{(2 N)}}(s) .
$$

In order to bound $M_{2 N}$, we need the following Cauchy's estimates.

Theorem 8.19 (Cauchy's Estimates). Suppose $f$ is holomorphic on a neighborhood of the ball $B=\left\{z \in \mathbb{C}:\left|z-z_{0}\right| \leq r\right\}$, then we have that

$$
\left|f^{(k)}\left(z_{0}\right)\right| \leq \frac{k !}{r^{k}} \cdot \sup _{z \in B}|f(z)| .
$$

Since $f(x)$ is a rational polynomial, we can extend the definition of $f(s)$ to the complex plane and the resulting function, which we also denote as $f(s)$. Since $\left\|\log \left(z_{0}\right)-\log \left(x_{0}\right)\right\|_{\infty} \leq \epsilon_{\text {comp }} \leq 0.01$, $M\left(y_{s, 0}\right)$ is invertible for $|s| \leq 1$. Hence $f$ is holomorphic on the unit ball on the complex plane around 0. Applying Theorem 8.19 with $r=1$, we have that

$$
M_{2 N} \leq(2 N) ! \cdot \operatorname{poly}(n) .
$$

Therefore, the error is bounded as

$$
\left|\sigma_{i, \mathrm{dis}}-\sigma_{i, \mathrm{cts}}\right| \leq \frac{\operatorname{poly}(n)}{2^{2 N}}
$$

which finishes the proof of the lemma. 
Lemma 8.20 (Error upper bound for $\theta^{\top} \theta$ ). Assume $\epsilon_{\text {comp }} \leq 0.01$. Let $\sigma_{i, \mathrm{cts}}$ and $\sigma_{i, \text { dis }}$ be defined as follows

$$
\sigma_{i, \mathrm{cts}}=\int_{0}^{1} \theta_{i, t}^{\top} \theta_{i, t} \mathrm{~d} t, \quad \sigma_{i, \mathrm{dis}}=\sum_{t \in \mathcal{T}} \omega_{t} \theta_{i, t}^{\top} \theta_{i, t}
$$

where $|\mathcal{T}|=N$. Then we have

$$
\left|\sigma_{i, \mathrm{dis}}-\sigma_{i, \mathrm{cts}}\right| \leq O\left(\operatorname{poly}(n) / 2^{2 N}\right)
$$

Lemma 8.21 (Error upper bound for $\alpha^{\top} \beta$ ). Assume $\epsilon_{\mathrm{comp}} \leq 0.01$. Let $\sigma_{i, \mathrm{cts}}$ and $\sigma_{i, \mathrm{dis}}$ be defined as follows

$$
\sigma_{i, \mathrm{cts}}=\int_{0}^{1} \int_{0}^{1} \beta_{i, s, t}^{\top} \alpha_{i, s, t} \mathrm{~d} s \mathrm{~d} t, \quad \sigma_{i, \mathrm{dis}}=\sum_{t \in \mathcal{T}} \sum_{s \in \mathcal{S}} \omega_{t} \omega_{s} \beta_{i, s, t}^{\top} \alpha_{i, s, t},
$$

where $|\mathcal{S}|=|\mathcal{T}|=N$. Then we have

$$
\left|\sigma_{i, \mathrm{dis}}-\sigma_{i, \mathrm{cts}}\right| \leq O\left(\operatorname{poly}(n) / 2^{2 N}\right) .
$$

Lemma 8.22 (Error upper bound for $\gamma^{\top} \gamma$ ). Assume $\epsilon_{\mathrm{comp}} \leq 0.01$. Let $\sigma_{i, \mathrm{cts}}$ and $\sigma_{i, \text { dis }}$ be defined as follows

$$
\begin{aligned}
\sigma_{i, \mathrm{cts}} & =\int_{0}^{1} \int_{0}^{1} \int_{0}^{1} \gamma_{i, s, t}^{\top} \gamma_{i, s^{\prime}, t} \mathrm{~d} s \mathrm{~d} s^{\prime} \mathrm{d} t \\
\sigma_{i, \mathrm{dis}} & =\sum_{t \in \mathcal{T}} \sum_{s \in \mathcal{S}} \sum_{s^{\prime} \in \mathcal{S}} \omega_{t} \omega_{s} \omega_{s^{\prime}} \gamma_{i, s, t}^{\top} \gamma_{i, s^{\prime}, t},
\end{aligned}
$$

where $|\mathcal{S}|=|\mathcal{T}|=N$. Then we have

$$
\left|\sigma_{i, \mathrm{dis}}-\sigma_{i, \mathrm{cts}}\right| \leq O\left(\operatorname{poly}(n) / 2^{2 N}\right) .
$$




\section{Appendix}

\section{A Perturbed Volumetric Center Cutting Plane Method}

In this section we present an overview of Vaidya's cutting plane method [Vai89a] and illustrate how our leverage score maintenance data structure in Section 5 implies a faster implementation in $O\left(n \mathrm{SO} \log (\kappa)+n^{3} \log (\kappa)\right)$ time. Formally, we prove the following Theorem 4.1 from Section 4 .

Theorem 4.1. Given a separation oracle for a convex set $K \subset \mathbb{R}^{n}$ that is contained in a box of radius $R$ and a parameter $\epsilon>0$, there is a cutting plane method that either computes a point in $K$ or proves that $K$ does not contain a ball of radius $\epsilon$ in $O\left(\left(n \mathrm{SO}+n^{3}\right) \log (\kappa)\right)$ time, where $\mathrm{SO}$ is the complexity of the separation oracle and $\kappa=n R / \epsilon$.

Our cutting plane method essentially replaces the leverage scores $\sigma$ in Vaidya's method by estimates $\widetilde{\sigma}$ from our leverage score maintenance data structure in Section 5 , which satisfies that $\|\widetilde{\sigma}-\sigma\|_{2} \leq 1 / \log ^{O(1)}(n)$. To justify the validility of such a replacement, we first give an overview of Vaidya's method.

Feasible region Vaidya's algorithm maintains a polytope $P^{(k)}=\left\{x \in \mathbb{R}^{n}: A^{(k)} x \geq b^{(k)}\right\} \subset \mathbb{R}^{n}$ with $m^{(k)}=O(n)$ constraints as the feasible region.

Volmetric barrier function and volumetric center Vaidya's algorithm maintains an approximate minimizer $z^{(k)} \in \mathbb{R}^{n}$, known as the volumetric center, of the volumetric barrier function:

$$
z^{(k)} \approx \arg \min _{x \in P^{(k)}} F^{(k)}(x) \quad \text { with } \quad F^{(k)}(x):=\frac{1}{2} \log \left(\operatorname{det}\left(A^{(k) \top}\left(S_{x}^{(k)}\right)^{-2} A^{(k)}\right)\right)
$$

where $s_{x}^{(k)}=A^{(k)} x-b^{(k)} \in \mathbb{R}^{m^{(k)}}$ is the slack and $S_{x}^{(k)} \in \mathbb{R}^{m^{(k)} \times m^{(k)}}$ is the diagonal matrix for $s_{x}^{(k)}$. Here $z^{(k)} \in \mathbb{R}^{n}$ serves as the query point to the separation oracle.

Leverage score Each constraint $i$ of $P^{(k)}$ is associated with a leverage score

$$
\sigma_{i}(z)=\left(\left(S_{x}^{(k)}\right)^{-1} A^{(k)}\left(A^{(k) \top}\left(S_{x}^{(k)}\right)^{-2} A^{(k)}\right)^{-1} A^{(k) \top}\left(S_{x}^{(k)}\right)^{-1}\right)_{i, i}, \forall i \in\left[m^{(k)}\right]
$$

which measures its relative importance (see preliminary for the definition). It is well-known that $0 \leq \sigma_{i}(z) \leq 1, \forall i \in\left[m^{(k)}\right]$ and $\sum_{i=1}^{m^{(k)}} \sigma_{i}(z)=n$. We denote by $\sigma$ and $\Sigma$ the vector and diagonal matrix of leverage scores respectively.

Updating $P^{(k)}$ according to leverage scores $P^{(k)}$ can be updated in two different ways. Parameters are chosen such that $F(z)-\min _{z \in \mathbb{R}^{n}} F(z)$ does not increased more than a multiplicative constant.

- Whenever the leverage score $\sigma_{i}(z)$ is smaller than some universal constant $c_{1}$, constraint $i$ is dropped and $z$ is updated by the Newton method below. As $\sum_{i} \sigma_{i}(z)=n$, this implies that the number of constraints is $\leq \frac{\sum_{i} \sigma_{i}}{c_{1}}=\frac{n}{c_{1}}=O(n)$.

- Otherwise, the separation oracle is queried at the volumetric center $z^{(k)}$ and returns a new separating hyperplane $a_{k}^{\top} x \geq b_{k}$. However, $P^{(k+1)}$ is not the intersection of $P^{(k)}$ and $a_{k}^{\top} x \geq b_{k}$. Instead, $a_{k}^{\top} x \geq b_{k}^{\prime}$ is added for some $b_{k}^{\prime} \leq b_{k}$ so that the leverage score of $a_{k}^{\top} x \geq b_{k}^{\prime}$ is $0.5\left(\delta c_{1}\right)^{1 / 2}$, where $\delta \geq 10^{3} c_{1}$ is another small universal constant. 
Updating $z^{(k)}$ via Newton-type method Upon adding or removing a constraint the volumetric center $z^{(k)} \in \mathbb{R}^{n}$ must be recomputed. As $z^{(k)}$ should minimize $F^{(k)}(x)$, we iteratively update $z^{(k)}$ via a Newton-type step:

$$
z^{\text {new }} \leftarrow z-\frac{1}{10} Q(z)^{-1} \nabla F(z)
$$

where $Q(z):=A^{\top} S_{z}^{-1} \Sigma S_{z}^{-1} A \in \mathbb{R}^{n \times n}$ can be shown to be a constant spectral approximation to the Hessian $\nabla^{2} F(z) \in \mathbb{R}^{n \times n}$. One can calculate $\nabla F(z)=A^{\top} S_{z}^{-1} \sigma \in \mathbb{R}^{n}$.

We perform this Newton step iteratively until $z^{\text {new }}$ is a good approximate minimizer of $F^{(k)}(x)$, i.e. $F(z)-\min _{z \in \mathbb{R}^{n}} F(z) \leq c_{2}$ for some small universal constant $c_{2}$. Theorem 1 in full version of [Vai89a] states that

$$
F\left(z^{\text {new }}\right)-\min _{z \in \mathbb{R}^{n}} F(z) \leq\left(1-\frac{1}{100}\right)\left(F(z)-\min _{z \in \mathbb{R}^{n}} F(z)\right)
$$

Since the decrease is multiplicative, this can be accomplished in only $O(1)$ many iterations.

\section{Performance guarantee of Vaidya's method}

Number of iterations required

Vaidya showed that after $T$ iterations, the volume of $P^{(k)}$ decreases by a factor of $c^{T-O(n \log (n))}$ for some constant $c$, i.e.

$$
\operatorname{vol}\left(P^{(k)}\right) \leq c^{T-O(n \log (n))} \operatorname{vol}\left(P^{(0)}\right)=c^{T-O(n \log (n))} R^{n} .
$$

Therefore in $T=O(n \log (n R / \epsilon))$ iterations, we have $\operatorname{vol}\left(P^{(k)}\right) \leq \epsilon^{O(n)}$ showing that $P^{(k)}$ does not contain a ball of radius $\epsilon$ and hence solving the feasibility problem.

\section{Running time per iteration}

As $\sum_{i} \sigma_{i}(z)=n$ and the leverage scores are maintained so that $\sigma_{i} \geq c_{1}$ always holds, the number of constraints is $\leq \frac{\sum_{i} \sigma_{i}}{c_{1}}=\frac{n}{c_{1}}=O(n)$. Thus all vectors and matrices above have dimension $O(n)$ and $O(n) \times O(n)$. Moreover, recall that only $O(1)$ steps of Newton method are needed within a iteartion of cutting plane.

Therefore in one iteration, the running time of Vaidya is $O\left(n^{2}\right)$ plus the time to compute $\sigma$ and to solve a linear system in $Q(z)^{-1}$ (from the Newton step), which naively requires $O\left(n^{\omega}\right)$ time. In the rest of this section we explain speed up these two bottlenecks using our leverage score maintenance data structure.

\section{A.1 Our faster implementation via leverage score maintenance}

We provide a faster implementation of Vaidya's method via our leverage score maintenance data structure, which efficiently updates leverage scores. Specifically, We design a data structure which, upon updates to the volumetric center $z$, maintains an estimate $\tilde{\sigma}$ of the leverage scores $\sigma$ in amortized $O\left(n^{2}\right)$ time (Theorem 5.1). Our error guarantee satisfies

$$
\|\widetilde{\sigma}-\sigma\|_{2} \leq 1 / \log O(1)(n) .
$$

To apply our data structure, we plug in $W=S_{z}^{-2}$ as the weight in Theorem 5.1. To establish the validity of our method, we show that conditions (1) and (2) required for our data structure are satisfied for sufficiently small parameters $c_{1}, \delta, c_{2}$. We then prove that Vaidya's performance guarantee is preserved in the presence of a small perturbation to the leverage score. 
Lemma A.1 (Condition 1 of data structure (Theorem 5.1)). For any constraint $a^{\top} x \geq b$ added or removed, let $s=a^{\top} z-b$ be its slack. We have

$$
\frac{1}{s^{2}} a a^{\top} \preceq 0.01 A^{\top} S_{z}^{-2} A .
$$

Proof. Let $H(z)=A^{\top} S_{z}^{-2} A$. Our goal is to show

$$
\frac{1}{s^{2}} a a^{\top} \preceq 0.01 H(z) .
$$

Recall that a constraint is removed when its leverage score is smaller than $c_{1}$ and added so that its leverage score is $\left(\delta c_{1}\right)^{1 / 2}$. In Vaidya's analysis, the only requirement on $c_{1}$ and $\delta$ is that $c_{1}, \delta$ are sufficiently small constants and $\delta \geq 10^{3} c_{1}$. Hence in either case, we can make the leverage score of $a^{\top} x \geq b$ smaller than 0.01 by choosing $c_{1}$ and $\delta$ small enough, i.e. the leverage score of $a^{\top} x \geq b$ satisfies

$$
\frac{1}{s^{2}} \cdot a^{\top} H(z)^{-1} a \leq 0.01
$$

Since $H(z)$ is PSD and the square root of a PSD matrix exists,

$$
\frac{1}{s^{2}} \cdot a^{\top} H(z)^{-1} a=\frac{1}{s^{2}} \cdot\left(H(z)^{-1 / 2} a\right)^{\top}\left(H(z)^{-1 / 2} a\right) \leq 0.01
$$

Note that the spectral norm of $\left(H(z)^{-1 / 2} a\right)\left(H(z)^{-1 / 2} a\right)^{\top}$ is $\left(H(z)^{-1 / 2} a\right)^{\top}\left(H(z)^{-1 / 2} a\right)$. Thus

$$
\frac{1}{s^{2}} H(z)^{-1 / 2} a^{\top} a H(z)^{-1 / 2}=\frac{1}{s^{2}}\left(H(z)^{-1 / 2} a\right)\left(H(z)^{-1 / 2} a\right)^{\top} \preceq 0.01 I .
$$

Multiplying by $H(z)^{1 / 2}$ on the both sides of the above equation, we have

$$
\frac{1}{s^{2}} a^{\top} a \preceq 0.01 \cdot H(z),
$$

thus we complete the proof.

Lemma A.2 (Condition 2 of data structure (Theorem 5.1)). Whenever the volumetric center $z \in \mathbb{R}^{n}$ is updated to $z^{\text {new }} \in \mathbb{R}^{n}$, we have

$$
\left\|\log \left(s_{z^{\text {new }}}\right)-\log \left(s_{z}\right)\right\|_{2} \leq 0.01
$$

Proof. First, we note that it suffices to show that

$$
\left\|\frac{s_{z^{\text {new }}}-s_{z}}{s_{z}}\right\|_{2} \leq 0.00001 .
$$

Indeed, this implies that

$$
(1-0.00001) s_{z^{\text {new }}} \leq s_{z} \leq(1+0.00001) s_{z^{\text {new }}} .
$$

Thus each coordinate of $\log \left(s_{z^{\text {new }}}\right)-\log \left(s_{z}\right)$ is bounded by $\log (1 \pm 0.00001)$.

Now using $\log ^{2}(1+t) \leq 2 t^{2}$ for $|t| \leq 0.01$, we have

$$
\left\|\log \left(s_{z^{\text {new }}}\right)-\log \left(s_{z}\right)\right\|_{2} \leq 2\left\|\frac{s_{z^{\text {new }}}-s_{z}}{s_{z}}\right\|_{2} \leq 0.01
$$


It then remains to prove $\left\|\frac{s_{z} \text { new }-s_{z}}{s_{z}}\right\|_{2} \leq 0.00001$

In Vaidya's work [Vai89a], they showed that

$$
\begin{aligned}
\left(z-z^{\text {new }}\right)^{\top} Q(z)\left(z-z^{\text {new }}\right) & =O\left(F(z)-\min _{z \in \mathbb{R}^{n}} F(z)\right) \\
& =O\left(\delta\left(\delta c_{1}\right)^{1 / 4}\right) \\
& =O\left(\delta^{5 / 4} c_{1}^{1 / 4}\right) .
\end{aligned}
$$

Recall that leverage scores are at least $c_{1}$, and $Q(z)=A^{\top} S_{z}^{-1} \widetilde{\Sigma} S_{z}^{-1} A$ is PSD. Thus

$$
\left(z^{\text {new }}-z\right)^{\top} Q(z)\left(z^{\text {new }}-z\right) \geq c_{1}\left(z^{\text {new }}-z\right)^{\top}\left(A^{\top} S_{z}^{-2} A\right)\left(z^{\text {new }}-z\right) .
$$

Moreover, note that $A\left(z^{\text {new }}-z\right)=s_{z^{\text {new }}}-s_{z}$ so

$$
\left(z^{\text {new }}-z\right)^{\top}\left(A^{\top} S_{z}^{-2} A\right)\left(z^{\text {new }}-z\right)=\left\|\frac{s_{z^{\text {new }}}-s_{z}}{s_{z}}\right\|_{2}^{2} .
$$

Combining all, we obtain

$$
\left\|\frac{s_{z^{\text {new }}}-s_{z}}{s_{z}}\right\|_{2}^{2}=O\left(\delta^{5 / 4} / c_{1}^{3 / 4}\right) .
$$

Having established the conditions of our data structure which maintains perturbed leverage scores, we argue that Vaidya's method tolerates small additive perturbations $o(1)$ in the leverage scores $^{3}$. Note that in the presence of such perturbations, both the procedure for updating $P^{(k)}$ and the Newton step are affected.

Lemma A.3 (Newton step). Assume the leverage scores $\sigma$ are replaced by estimate $\widetilde{\sigma}$, where $\| \widetilde{\sigma}-$ $\sigma \|_{2} \leq 1 / \log ^{O(1)}(n)$ in Vaidya's method. Then Newton step still requires only $O(1)$ many iterations so that $F(z)-\min _{z \in \mathbb{R}^{n}} F(z) \leq c_{2}$ for some small constant $c_{2}$.

Proof. For the Newton step, let $\widetilde{\Sigma}$ be the diagonal matrix of $\widetilde{\sigma}$. Vaidya's Newton step is modified as

$$
z^{\text {new }} \leftarrow z-\frac{1}{10}\left(A^{\top} S_{z}^{-1} \widetilde{\Sigma} S_{z}^{-1} A\right)^{-1} A^{\top} S_{z}^{-1} \widetilde{\sigma}
$$

As only $\Sigma$ and $\sigma$ are changed, this amounts to a small difference in the convergence rate, i.e.

$$
F\left(z^{\text {new }}\right)-\min _{z \in \mathbb{R}^{n}} F(z) \leq\left(1-\frac{1}{101}\right)\left(F(z)-\min _{z \in \mathbb{R}^{n}} F(z)\right)+O\left(\|\widetilde{\sigma}-\sigma\|_{2}^{2}\right) .
$$

Recall that our goal is $F(z)-\min _{z \in \mathbb{R}^{n}} F(z) \leq c_{2}$ for a small universal constant $c_{2}$. Therefore our modified Newton step still requires only $O(1)$ many iterations as long as $\|\widetilde{\sigma}-\sigma\|_{2}^{2}=o(1)$.

Lemma A.4 (Convergence rate). Assume the leverage scores $\sigma$ are replaced by estimate $\tilde{\sigma}$, where $\|\widetilde{\sigma}-\sigma\|_{2} \leq 1 / \log ^{O(1)}(n)$ in Vaidya's method.

Then Vaidya's convergence guarantee still holds: after $T$ iterations, the volume of $P^{(k)}$ decreases by a factor of $c^{T-O(n \log (n))}$ for some constant $c$, i.e.

$$
\operatorname{vol}\left(P^{(k)}\right) \leq c^{T-O(n \log (n))} \operatorname{vol}\left(P^{(0)}\right)=c^{T-O(n \log (n))} R^{n} .
$$

\footnotetext{
${ }^{3}$ In fact, Vaidya's method would survive even if the perturbation is a sufficiently small constant.
} 
Proof. The proof of Vaidya's convergence lemma essentially depends on the fact that leverage scores are at least $c_{1}$ and at most $0.5\left(\delta c_{1}\right)^{1 / 2}$.

Note that $P^{(k)}$ is updated by dropping constraint $i$ if $\sigma_{i}(z) \geq c_{1}$, or adding constraint $i$ s.t. $\sigma_{i}(z)=0.5\left(\delta c_{1}\right)^{1 / 2}$. The purpose of the constraint adding and dropping is to make sure the leverage score of all constraints are $\Theta(1)$. Hence, we can use any constant approximation to leverage score. In particular, an additive $o(1)$ pertubation in the leverage score can be absorbed by scaling $c_{1}, \delta$ slightly.

Now we are ready to bound the running time of our modification of Vaidya's cutting plane method and complete the proof of Theorem 4.1.

Proof of Theorem 4.1. By Lemma A.4, in $T=O(n \log (n R / \epsilon))=O(n \log (\kappa))$ iterations, we have $\operatorname{vol}\left(P^{(k)}\right) \leq \epsilon^{O(n)}$ showing that $P^{(k)}$ does not contain a ball of radius $\epsilon$. Thus the number of calls to the separation oracle is $O(n \log (\kappa))$. We next analyze the runtime per iteration.

By Lemma A.3, we still only need $O(1)$ Newton steps. Thus, as argued at the end of last subsection, the per-iteration running time is $O\left(n^{2}\right)$ plus the time to compute $\sigma$ and to solve a linear system in $Q(z)^{-1}$. We argue that both of these two tasks can be accomplished in amortized $O\left(n^{2}\right)$ time.

For $\sigma$, we instead use its estimate $\widetilde{\sigma}$ output by our leverage score maintenance data structure (Theorem 5.1). By Lemmas A.1 and A.2, the conditions of the data structure are satisfied. Hence we can update $\widetilde{\sigma}$ in amortized $O\left(n^{2}\right)$ time.

Solving a linear system in $Q(z)^{-1}$, as pointed out in Theorem 31 of LSW [LSW15], can be done by inverse maintenance. Using the inverse maintenance procedure in [CLS19], this can also be done in amortized $O\left(n^{2}\right)$ time (see Theorem B.4). 


\section{B Modified Projection Maintenance}

Theorem B.1 ([CLS19, Appendix]). Let $t_{k}=\mathcal{T}_{\text {mat }}(m, m, k)$ denote the time to multiply a $m \times m$ and a $m \times k$ matrix. Given a matrix $A \in \mathbb{R}^{m \times n}$ with $m \geq n$, and $k^{*} \in[m]$, there is a deterministic data structure that approximately maintains the projection matrices $\sqrt{W} A\left(A^{\top} W A\right)^{-1} A^{\top} \sqrt{W}$ and the inverse matrices $\left(A^{\top} W A\right)^{-1}$ for positive diagonal matrices $W$ through the following operations:

- $\operatorname{Initialize}(A, w, \epsilon)$ : Initialize the data structure of the matrix $A$, the weight $w$ and the target accuracy $\epsilon \in(0,1 / 4)$ in $m^{\omega+o(1)}$ time.

- $\operatorname{Update}(w)$ : Output a vector $v \in \mathbb{R}^{m}$ along with $\left(A^{\top} V A\right)^{-1}$ and $\sqrt{V} A\left(A^{\top} V A\right)^{-1} A^{\top} \sqrt{V}$ such that for all $i$,

$$
(1-\epsilon) v_{i} \leq w_{i} \leq(1+\epsilon) v_{i}
$$

- $\operatorname{Insert}\left(a, w_{a}\right)$ : Insert a column a into $A$, a weight $w_{a}$ into $w$ in $O\left(m^{2}\right)$ time.

- $\operatorname{Delete}\left(a, w_{a}\right)$ : Delete a column a from $A$ and its corresponding weight $w_{a}$ from $w$ in $O\left(m^{2}\right)$ time.

Suppose that the number of columns is $O(m)$ during the whole algorithm and that for any call of UPDATE, we have

$$
\sum_{i=1}^{m}\left(\log w_{i}-\log w_{i}^{(o l d)}\right)^{2} \leq C^{2}
$$

where $w$ is the input of call, $w^{(\text {old })}$ is the weight before the call. Then, the amortized expected time per call of $\operatorname{UPDATE}(w)$ is

$$
O\left(t_{k^{*}}+(C / \epsilon) \cdot\left(\frac{t_{k^{*}}^{2}}{k^{*}}+\sum_{i=k^{*}}^{m} \frac{t_{i}^{2}}{i^{2}}\right)^{1 / 2} \cdot \log m\right)
$$

We will use this theorem with difference algorithms for rectangular matrix multiplication. Recall that it takes $O\left(m^{2} \log ^{2} m\right)$ time to multiply an $m \times m$ and an $m \times m^{0.17}$ matrix. By splitting the matrix into blocks (See e.g. [CLS19, Lemma A.5]), one can check it takes

$$
t_{k}:=O\left(m^{2} \log ^{2} m+k^{\frac{\omega-2}{1-\alpha}} m^{2-\frac{\alpha(\omega-2)}{1-\alpha}} \log ^{2} m\right)
$$

time to multiply a $m \times m$ and a $m \times k$ matrix with $\alpha=0.17$. Using this and putting $k^{*}=m^{\alpha}$, we have

$$
\begin{aligned}
\left(\frac{t_{k^{*}}^{2}}{k^{*}}+\sum_{i=k^{*}}^{m} \frac{t_{i}^{2}}{i^{2}}\right)^{1 / 2} & \leq \frac{m^{2} \log ^{2} m}{m^{\alpha / 2}}+m^{2-\frac{\alpha(\omega-2)}{1-\alpha}} \log ^{2} m\left(\sum_{i=k^{*}}^{m} \frac{i^{2 \frac{\omega-2}{1-\alpha}}}{i^{2}}\right)^{1 / 2} \\
& =m^{2-\alpha / 2} \log ^{2} m+m^{2-\frac{\alpha(\omega-2)}{1-\alpha}} \log ^{2} m \cdot O\left(m^{-\frac{\alpha}{2}\left(1-2 \frac{\omega-2}{1-\alpha}\right)}\right) \\
& =O\left(m^{2-\alpha / 2} \log ^{2} m\right)
\end{aligned}
$$

Hence, applying Theorem B.1 with $k^{*}=m^{0.17}$, we have the following Theorem

Theorem B.2. There is a variant of the data structure in Theorem B.1 where the amortized time per call of $\operatorname{UpDATE}(w)$ is

$$
O\left(m^{2} \log ^{2} m+C m^{2} \epsilon^{-1} m^{-0.085}\right) .
$$


Unfortunately, this version still have extra $\log ^{O(1)} m$ terms in the runtime. To get the $m^{2}$ time, we use the following lemma:

Lemma B.3. For any $c>0$, it takes

$$
O\left(m^{2}+\frac{m^{2} r^{0.4}}{\log ^{c} m}\right)
$$

time to multiply $m \times r$ and $r \times m$ matrices.

Proof. If $r>m^{\frac{0.38}{0.39}}$, we simply multiply it using a $m^{2.38}$ time square matrix multiplication algorithm. This is faster than $m^{2} r^{0.39} \leq O\left(\frac{m^{2} r^{0.4}}{\log ^{c} m}\right)$. If $r<\log ^{2 c} m$, we simply use (3) in Theorem 3.8 which takes $O\left(m^{2}\right)$ time. Hence, we can assume $\log ^{2 c} m<r<m^{\frac{0.38}{0.39}}$.

Let $k=\frac{r}{\log ^{c / 0.4} m}$. We can view the problem as multiplying a $\frac{m}{k} \times \frac{r}{k}$ and a $\frac{r}{k} \times \frac{m}{k}$ block matrices and each block has size $k \times k$ size.

Note that

$$
\frac{m}{k}=\frac{m \log ^{c / 0.4} m}{r}=m^{\Omega(1)} \quad \text { and } \quad \frac{r}{k}=\log ^{O(1)} m
$$

Hence, (3) in Theorem 3.8 shows that the total cost is $O\left(\left(\frac{m}{k}\right)^{2}\right)$ many block matrix multiplication and each takes $O\left(k^{2.4}\right)$ time. Therefore, the total cost is

$$
O\left(\left(\frac{m}{k}\right)^{2} \times k^{2.4}\right)=O\left(m^{2} k^{0.4}\right)=O\left(\frac{m^{2} r^{0.4}}{\log ^{c} m}\right) .
$$

Now, applying Theorem B.1 with $k^{*}=\log ^{O(1)} m$, we have

Theorem B.4. For any $c>0$, there is a variant of the data structure in Theorem B.1 with the amortized time per call of $\operatorname{UPDATE}(w)$ is

$$
O\left(m^{2}+\frac{C m^{2}}{\epsilon \log ^{c} m}\right)
$$




\section{Cutting Plane Method for Convex Minimization and Saddle Point Problems}

We show in this section that cutting plane methods can be applied to not only convex minimization, but also the more general problem of computing a saddle point in a convex-concave game with essentially the same guarantee.

\section{C.1 Notations and definitions}

For a convex set $\mathcal{X} \subseteq \mathbb{R}^{n}$, the interior of $\mathcal{X}$, denoted as int $(\mathcal{X})$, is the subset of points in $\mathcal{X}$ that has a small neighborhood fully contained inside $\mathcal{X}$. We denote by vol $(\mathcal{X})$ the volume of $\mathcal{X}$. For any vector $x$ and $r>0$, we use $B(x, r)$ to denote the $\ell_{2}$ ball of radius $r$ centered at $x$, i.e. $B_{\infty}(x, r)=\left\{y:\|x-y\|_{2} \leq r\right\}$. Similarly, $B_{\infty}(x, r)=\left\{y:\|x-y\|_{\infty} \leq r\right\}$. For any set $K$, we denote $B(K,-\epsilon)$ to denote the set $\{x: B(x, \epsilon) \subset K\}$.

\section{C.2 Convex minimization}

Using a standard reduction of convex minimization to the feasiblity problem ([Nem94] and Theorem 42 of [LSW15]), we can minimize a convex function with $O(n \log \kappa)$ subgradient oracle calls and $O\left(n^{3} \log \kappa\right)$ time. This improves over the previous best of $O\left(n^{3} \log ^{O(1)} \kappa\right)$ [LSW15]. Since $\kappa$ can be exponential in certain applications, this allows us to obtain significantly faster algorithms (see e.g. subsection D.3).

Theorem C.1. Let $f$ be a convex function on $\mathbb{R}^{n}$ and $S$ be a convex set that contains a minimizer of $f$. Suppose we have a subgradient oracle for $f$ with cost $\mathcal{T}$ and $S \subset B_{\infty}(0, R)$. Using $B_{\infty}(0, R)$ as the initial polytope for our Cutting Plane Method, for any $0<\alpha<1$, we can compute $x \in S$ such that $f(x)-\min _{y \in S} f(y) \leq \alpha\left(\max _{y \in S} f(y)-\min _{y \in S} f(y)\right)$, with high probability in $n$ and with a running time of

$$
O\left(\mathcal{T} \cdot n \log (\kappa)+n^{3} \log (\kappa)\right)
$$

where $\kappa=n \gamma / \alpha$ and $\gamma=R / \operatorname{minwidth}(S)$. Here the minwidth of $S$ is defined by

$$
\operatorname{minwidth}(S):=\min _{a \in \mathbb{R}^{n}:\|a\|_{2}=1}\left(\max _{y \in S} a^{\top} y-\min _{y \in S} a^{\top} y\right)
$$

\section{C.3 Convex-concave games}

In this subsection we show that a similar guarantee holds for solving convex-concave games. Much of the materials in this section are modified from [Nem95, Lecture 5]. For completeness, we will explain both the standard theory and the various changes needed for our promised runtime.

In the convex-concave game, we are asked to solve

$$
\min _{x \in \mathcal{X}} \max _{y \in \mathcal{Y}} f(x, y)
$$

where $\mathcal{X} \in \mathbb{R}^{n}, \mathcal{Y} \in \mathbb{R}^{m}$ are convex sets and $f(x, y): \mathcal{X} \times \mathcal{Y} \rightarrow \mathbb{R}$ is a continuous function that is convex in $x \in \mathcal{X}$ and concave in $y \in \mathcal{Y}$. Von Neumann's minimax theorem states that

$$
\min _{x \in \mathcal{X}} \max _{y \in \mathcal{Y}} f(x, y)=\max _{y \in \mathcal{Y}} \min _{x \in \mathcal{X}} f(x, y),
$$


and that all solutions to the LHS are solutions to the RHS, and vice versa. Any solution $\left(x^{*}, y^{*}\right)$ to this problem is called a saddle point, and satisifes $f\left(x, y^{*}\right) \geq f\left(x^{*}, y^{*}\right) \geq f\left(x^{*}, y\right)$ for any $(x, y) \in$ $\mathcal{X} \times \mathcal{Y}$.

We will be interested in computing an $\epsilon$-saddle point which we define in the following. Define $\bar{f}(x):=\max _{y \in \mathcal{Y}} f(x, y)$ and $\underline{f}(y)=\min _{x \in \mathcal{X}} f(x, y)$. The minimax theorem is equivalent to

$$
\min _{x \in \mathcal{X}} \bar{f}(x)=\min _{x \in \mathcal{X}} \max _{y \in \mathcal{Y}} f(x, y)=\max _{y \in \mathcal{Y}} \min _{x \in \mathcal{X}} f(x, y)=\max _{y \in \mathcal{Y}} \underline{f}(y),
$$

and it is immediate that the set of saddle points $S^{*}(f)$ is exactly the direct product of the optimal solutions to $\bar{f}: \mathcal{X} \rightarrow \mathbb{R}$ and $\underline{f}: \mathcal{Y} \rightarrow \mathbb{R}:$

$$
S^{*}(f)=\arg \max _{x \in \mathcal{X}} \bar{f}(x) \times \arg \min _{y \in \mathcal{Y}} \underline{f}(y) .
$$

Since certifying the values of $f(x, y)$ around the boundary of $\mathcal{X}$ and $\mathcal{Y}$ is quite difficult under the black-box setting, our definition of $\epsilon$-saddle point ignores small portion of the domain around the boundary.

Definition C.2 ( $\epsilon$-Saddle Point). Consider a convex-concave game with convex sets $\mathcal{X} \subseteq B(0, R) \subset$ $\mathbb{R}^{n}, \mathcal{Y} \subseteq B(0, R) \subset \mathbb{R}^{m}$, and L-Lipschitz function $f(x, y): \mathcal{X} \times \mathcal{Y} \rightarrow \mathbb{R}$ that is convex in $x$ and concave in $y$. Given $\epsilon \in(0,1)$, a pair $(x, y) \in \mathcal{X} \times \mathcal{Y}$ is called an $\epsilon$-saddle point, if

$$
\bar{f}(x)-\underline{f}(y) \leq \epsilon L R \text {. }
$$

It follows from (13) that the LHS of (14) is

$$
\bar{f}(x)-\underline{f}(y)=\left(\bar{f}(x)-\min _{x^{\prime} \in \mathcal{X}} \bar{f}\left(x^{\prime}\right)\right)+\left(\max _{y^{\prime} \in \mathcal{Y}} \underline{f}\left(y^{\prime}\right)-\underline{f}(y)\right) .
$$

In what follows we assume access to a first-order oracle which, given $(x, y) \in \operatorname{int}(\mathcal{X} \times \mathcal{Y})$, returns the subgradient vector

$$
g(x, y)=\left(\nabla_{x} f(x, y),-\nabla_{y} f(x, y)\right) \in \mathbb{R}^{n} \times \mathbb{R}^{m} .
$$

The crucial property of this vector is as follows:

Lemma C.3 (Convex-Concave Property). Let $f: \mathcal{X} \times \mathcal{Y} \rightarrow \mathbb{R}$ be convex in $x$ and concave in $y$. Let $g: \mathcal{X} \times \mathcal{Y} \rightarrow \mathbb{R}^{n+m}$ be defined as in Eq. (15). For any $z=(x, y) \in \mathcal{X} \times \mathcal{Y}$ and $z^{\prime}=\left(x^{\prime}, y^{\prime}\right) \in$ $\operatorname{int}(\mathcal{X} \times \mathcal{Y})$, we have

$$
\left(z^{\prime}-z\right)^{\top} g\left(z^{\prime}\right) \geq f\left(x^{\prime}, y\right)-f\left(x, y^{\prime}\right) .
$$

In particular, if $z=(x, y)$ is a saddle point of $f$, then we have

$$
\left(z^{\prime}-z\right)^{\top} g\left(z^{\prime}\right) \geq 0 \text {. }
$$

Proof. Since $f$ is convex in $x$ and concave in $y$, we have

$$
f\left(x, y^{\prime}\right)-f\left(x^{\prime}, y^{\prime}\right) \geq\left(x-x^{\prime}\right)^{\top} \nabla_{x} f\left(x^{\prime}, y^{\prime}\right) \quad \text { and } \quad f\left(x^{\prime}, y\right)-f\left(x^{\prime}, y^{\prime}\right) \leq\left(y-y^{\prime}\right)^{\top} \nabla_{y} f\left(x^{\prime}, y^{\prime}\right) .
$$

It follows that

$$
f\left(x^{\prime}, y\right)-f\left(x, y^{\prime}\right) \leq\left(z^{\prime}-z\right)^{\top} g\left(z^{\prime}\right)
$$

which proves the first part of the lemma. For the second part, simply notice that if $z=(x, y)$ is a saddle point, we have

$$
f\left(x^{\prime}, y\right) \geq f(x, y) \geq f\left(x, y^{\prime}\right)
$$




\section{C.4 Applying cutting plane method to convex-concave games}

In the cutting plane framework, we maintain a polytope $P^{(k)} \subseteq \mathbb{R}^{n+m}$ that contains a saddle point $\left(x^{*}, y^{*}\right)$ of $f$. The algorithm initially starts with the polytope $P^{(0)}:=B_{\infty}(0, R) \supset \mathcal{X} \times \mathcal{Y}$.

In each iteration, we call the first-order oracle at a certain point $z^{(k)}=\left(x^{(k)}, y^{(k)}\right) \in P^{(k)}$ depending on $P^{(k)}$. If $z^{(k)} \notin \mathcal{X} \times \mathcal{Y}$ is not feasible, then we obtain a supporting hyperplane to separate $z^{(k)}$ from the feasible region $\mathcal{X} \times \mathcal{Y}$; and if $z^{(k)} \in \mathcal{X} \times \mathcal{Y}$ is feasible, Lemma C.3 shows that any saddle point $\left(x^{*}, y^{*}\right)$ lies in the half-space $H^{(k)}:=\left\{z \in \mathbb{R}^{n+m}:\left(z-z^{(k)}\right)^{\top} g\left(z^{(k)}\right) \leq 0\right\}$ and hence $\left(x^{*}, y^{*}\right) \in P^{(k)} \cap H^{(k)}$. The algorithm continues by choosing $P^{(k+1)}$ which contains $P^{(k)} \cap H^{(k)}$.

For simplicity, we extend the gradient function $g$ as follows:

$$
\widehat{g}_{\beta}(x, y)= \begin{cases}\left(\nabla_{x} f(x, y),-\nabla_{y} f(x, y)\right) & \text { if }(x, y) \in \operatorname{int}(\mathcal{X} \times \mathcal{Y}) \\ n(x, y) & \text { otherwise }\end{cases}
$$

where $n(x, y)$ is some vector of length $\beta>L$ such that $\mathcal{X} \times \mathcal{Y} \subset\left\{z \in \mathbb{R}^{n+m}:(z-(x, y))^{\top} n(x, y) \leq\right.$ $0\}$. The cutting plane framework is given in Algorithm 6 .

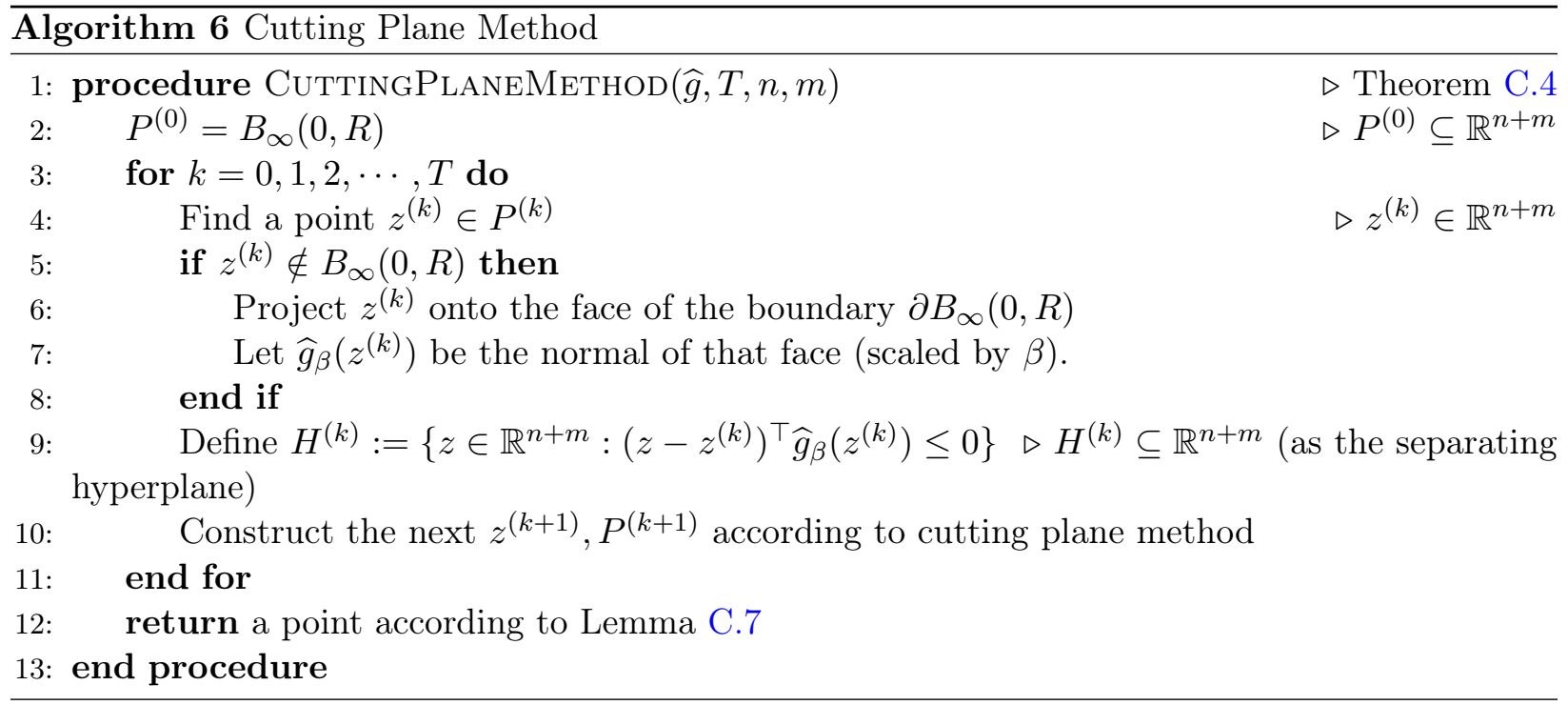

Theorem C.4. Let $c<1$ be a universal constant. For $T \geq n+m$, we can find $z^{(k)} \in \mathbb{R}^{n+m}$ and $P^{(k)} \subseteq \mathbb{R}^{n+m}$ in total time $O\left((n+m)^{2} T\right)$ with high probability in $n+m$ such that

$$
\operatorname{vol}\left(P^{(T)}\right) \leq c^{T-O(n+m) \log (n+m)} \operatorname{vol}\left(P^{(0)}\right) .
$$

Furthermore, $P^{(T)} \subset \bigcap_{k \in I} H^{(k)} \cap B_{\infty}(0, R)$ with $|I|=O(n)$.

Proof. By Lemma C.3, any saddle point lies in $H^{(k)}$ showing that it is indeed a separating hyperplane. With this separation oracle, we apply our faster implementation of Vaidya's cutting plane method from section 4 .

The second part of the lemma follows from the fact that this method always maintains a polytope with $O(n)$ constraints. For the first part, note that this just paraphases Lemma A.4.

\section{C.5 Cutting plane method for convex-concave games: generating solutions}

Recall that for minimizing a convex function $f(z)$, one can simply output the best $z^{(k)}$ found in all iterations. The argument here is that as long as the volume $\operatorname{vol}\left(P^{(T)}\right)$ is small enough, then in some 
iteration $k$, a point close to the optimal solution $z^{*}$ gets removed from $P^{(k)}$ which indicates that $f\left(z^{(k)}\right)$ is also close to optimal. For the convex-concave game, however, such a naive approach of outputting the "best" $z^{(k)}$ would fail as illustrated by [Nem95, Section 5.3]. The crucial difference here in the convex-concave game is that although we have an objective function $f$, but we are not interested in optimizing it. Instead, we are interested in finding an $\epsilon$-saddle point $(x, y)$ that satisfies (14).

The correct idea is to output some convex combination of all $z^{(k)}$ 's that does converge to the saddle point $\left(x^{*}, y^{*}\right) \in \mathcal{X} \times \mathcal{Y}$ which we describe in the following.

Assume that we have performed $T$ steps of the cutting plane method.

Definition C.5 (Gap function). Let I be the set of all constraints in $P^{(k)} \cap B_{\infty}(0, R)$. If the $k^{\text {th }}$ constraint comes from $B_{\infty}(0, R)$, denote by $z^{(k)}$ the center of the face and $g^{(k)}$ the vector normal to the face scaled by $\beta$. Otherwise, $z^{(k)}$ and $g^{(k)}$ denote the query point $z^{(k)}$ of the oracle and its output $\widehat{g}_{\beta}\left(z^{(k)}\right)$. The gap function $\gamma$ is defined as

$$
\gamma(z)=\min _{k \in I}\left(z^{(k)}-z\right)^{\top} g^{(k)}:=\min _{k \in I} \gamma^{(k)}(z) .
$$

Notice that $\gamma(z)$ is concave as it is the minimum of affine functions.

Now we show that if $\gamma(z)$ is small for all $z$, then we can form a good approximation to the saddle point set from $z^{(k)}$. We first prove the following lemma which states that the maximum of $\gamma(z)$ is given by some certain convex combination of $\gamma^{(k)}(z)$.

Lemma C.6 (Optimal Lagrange Multipliers). Define $\Delta_{I}$ to be the simplex of all convex combinations of $I$, i.e., $\Delta_{I}:=\left\{\lambda \in \mathbb{R}^{I} \mid \lambda_{k} \geq 0, \sum_{k \in I} \lambda_{k}=1\right\}$. Then there exist optimal Lagrange multipliers $\lambda^{*}=\left\{\lambda_{k}^{*}\right\}_{k \in I} \in \Delta$ such that

$$
\sum_{k \in I} \lambda_{k}^{*} \cdot \gamma^{(k)}\left(z^{\prime}\right)=\max _{z \in \mathbb{R}^{n+m}} \gamma(z) \quad \forall z^{\prime} \in \mathbb{R}^{n+m} .
$$

Furthermore, for any $0<\eta<1 / 2$, we can find $\lambda \in \Delta$ such that

$$
\sum_{k \in I} \lambda_{k} \cdot \gamma^{(k)}\left(z^{\prime}\right) \leq \max _{z \in \mathbb{R}^{n+m}} \gamma(z)+\eta \beta R \quad \forall z^{\prime} \in B_{\infty}(0, R)
$$

in time $O\left((n+m)^{\omega+o(1)} \log ((n+m) / \eta)\right.$.

Proof. By the minimax theorem, we have

$$
\max _{z \in \mathbb{R}^{n+m}} \gamma(z):=\max _{z \in \mathbb{R}^{n+m}} \min _{k \in I} \gamma^{(k)}(z)=\max _{z} \min _{\lambda \in \Delta} \sum_{k \in I} \lambda_{k} \cdot \gamma^{(k)}(z)=\min _{\lambda \in \Delta} \max _{z \in \mathbb{R}^{n+m}} \sum_{k \in I} \lambda_{k} \cdot \gamma^{(k)}(z),
$$

where we used the fact that $\sum_{k \in I} \lambda_{k} \cdot \gamma^{(k)}(z)$ is bilinear in $\lambda$ and $z$. The first part of the lemma follows from the fact that the affine function $\sum_{k \in I} \lambda_{k} \cdot \gamma^{(k)}(z)$ has a maximium over $z \in \mathbb{R}^{n+m}$ if and only if it is a constant function. Note that this happens precisely when $\sum_{k \in I} \lambda_{k} \cdot g^{(k)}=0$.

The second part of the lemma follows from the observation that " $\sum_{k \in I} \lambda_{k} \cdot \gamma^{(k)}(z)$ is a constant function" is a linear constraint over $\lambda$. Therefore, finding $\lambda \in \Delta$ that forms a constant function with smallest possible value can be captured by the following linear program (in $\lambda$ ): 


$$
\begin{aligned}
\max _{\lambda} & \sum_{k \in I} z^{(k)} \cdot g^{(k)} \lambda_{k} \\
\text { s.t. } & \sum_{k \in I} g^{(k)} \lambda_{k}=0 \\
& \sum_{k \in I} \lambda_{k}=1 \\
& \lambda_{k} \geq 0, \forall k \in I .
\end{aligned}
$$

Using a recent LP solver from Theorem 2.1 in [CLS19], in $O\left((n+m)^{\omega+o(1)} \log (1 / \delta)\right)$ time we can output an approximate solution $\lambda_{k}$ satisfying

$$
\begin{aligned}
\sum_{k \in I} z^{(k)} \cdot g^{(k)} \lambda_{k} & \geq \sum_{k \in I} z^{(k)} \cdot g^{(k)} \lambda_{k}^{*}-\delta \max _{k \in I}\left|z^{(k)} \cdot g^{(k)}\right| \\
& \geq \sum_{k \in I} z^{(k)} \cdot g^{(k)} \lambda_{k}^{*}-\delta \sqrt{n+m} R \beta \\
& =\max _{z \in \mathbb{R}^{n+m}} \gamma(z)-\delta \sqrt{n+m} R \beta
\end{aligned}
$$

and

$$
\left\|\sum_{k \in I} g^{(k)} \lambda_{k}\right\|_{1} \leq \delta\left(\sum_{k}\left\|g_{k}\right\|_{1}\right) \leq \delta \sqrt{n+m} \beta .
$$

where we used $\left\|g^{(k)}\right\|_{2} \leq \max (\beta, L)=\beta$ and $z^{(k)} \in B_{\infty}(0, R)$. Now for $z^{\prime} \in B_{\infty}(0, R)$,

$$
\begin{aligned}
& \sum_{k \in I} \lambda_{k} \cdot \gamma^{(k)}\left(z^{\prime}\right) \\
= & \sum_{k \in I} z^{(k)} \cdot g^{(k)} \lambda_{k}+\left(\sum_{k \in I} g^{(k)} \lambda_{k}\right) \cdot z^{\prime} \\
\geq & \max _{z \in \mathbb{R}^{n+m}} \gamma(z)-\delta \sqrt{n+m} R \beta-(\delta \sqrt{n+m} \beta)(\sqrt{n+m} R) \\
\geq & \max _{z \in \mathbb{R}^{n+m}} \gamma(z)-2(\delta(n+m) \beta R)
\end{aligned}
$$

Our result then follows by taking $\eta=2 \delta(n+m)$.

Now given the notion of approximate multipliers (17), we prove the following lemma.

Lemma C.7 (Convex Combination Provides a Good Solution). Given $\lambda$ satisfying

$$
\sum_{k \in I} \lambda_{k} \cdot \gamma^{(k)}\left(z^{\prime}\right) \leq 9 \eta \beta R(n+m)^{1 / 2} \quad \forall z^{\prime} \in B_{\infty}(0, R)
$$

with $\eta<1$. Assume that $\beta \geq 3 \sqrt{n+m} \cdot$ L. Let

$$
\widehat{z}=(\widehat{x}, \widehat{y})=\left(\sum_{k \in J} \lambda_{k} \cdot z^{(k)}\right) /\left(\sum_{k \in J} \lambda_{k}\right)
$$


where $J=\left\{k \in I: z^{(k)} \in \mathcal{X} \times \mathcal{Y}\right\}$. Then $\widehat{z}$ is feasible, i.e. $\widehat{z} \in \mathcal{X} \times \mathcal{Y}$, and we have

$$
\bar{f}(\widehat{x})-\underline{f}(\widehat{y}) \leq 18 \eta \beta R(n+m)^{1 / 2} .
$$

Proof. Since $z^{(k)} \in \mathcal{X} \times \mathcal{Y}$ for each $k \in J$, it follows from convexity that $\widehat{z} \in \mathcal{X} \times \mathcal{Y}$. For any point $z=(x, y) \in \mathcal{X} \times \mathcal{Y}$, we have $\widehat{g}_{\beta}\left(z^{(k)}\right)=g\left(z^{(k)}\right)$ and from Lemma C.3, for any $k \in J$

$$
\gamma^{(k)}(z):=\left(z^{(k)}-z\right)^{\top} \cdot g\left(z^{(k)}\right) \geq f\left(x^{(k)}, y\right)-f\left(x, y^{(k)}\right) .
$$

Let $\widehat{\lambda}_{k}=\lambda_{k} /\left(\sum_{k \in J} \lambda_{k}\right)$. Taking weighted sum of these inequalities, we have

$$
\sum_{k \in J} \widehat{\lambda}_{k} \cdot \gamma^{(k)}(z) \geq \sum_{k \in J} \widehat{\lambda}_{k} \cdot f\left(x^{(k)}, y\right)-\sum_{k \in J} \widehat{\lambda}_{k} \cdot f\left(x, y^{(k)}\right) \geq f(\widehat{x}, y)-f(x, \widehat{y}),
$$

where the last inequality follows from the fact that $f$ is convex in $x$ and concave in $y$. Taking the maximum over $z \in \mathcal{X} \times \mathcal{Y}$ on both sides, we have

$$
\begin{aligned}
\bar{f}(\widehat{x})-\underline{f}(\widehat{y}) & \leq \max _{z \in \mathcal{X} \times \mathcal{Y}} \sum_{k \in J} \widehat{\lambda}_{k} \cdot \gamma^{(k)}(z) \\
& \leq \max _{z \in \mathcal{X} \times \mathcal{Y}} \sum_{k \in I} \widehat{\lambda}_{k} \cdot \gamma^{(k)}(z) \\
& =\frac{1}{\sum_{k \in J} \lambda_{k}} \max _{z \in \mathcal{X} \times \mathcal{Y}} \sum_{k \in I} \lambda_{k} \cdot \gamma^{(k)}(z) \\
& \leq \frac{1}{\sum_{k \in J} \lambda_{k}} 9 \eta \beta R(n+m)^{1 / 2}
\end{aligned}
$$

where the second inequality uses $\gamma^{(k)}(z) \geq 0$ for all $k \notin J$ and $z \in \mathcal{X} \times \mathcal{Y}$ because $\mathcal{X} \times \mathcal{Y} \subset\{z \in$ $\left.\mathbb{R}^{n+m}:(z-(x, y))^{\top} n(x, y) \leq 0\right\}$, the third inequality follows from the assumption.

Now we bound $\sum_{k \in J} \lambda_{k}$. For $k \in J$, because $f$ is $L$-Lipschitz and $\left\|z^{(k)}\right\|_{2} \leq \sqrt{n+m} R$

$$
\gamma^{(k)}(0)=z^{(k) \top} \widehat{g}_{\beta}\left(z^{(k)}\right) \geq-\sqrt{n+m} L R .
$$

For $k \in I \backslash J$, we claim that

$$
\gamma^{(k)}(0)=z^{(k) \top} n\left(z^{(k)}\right) \geq \beta R
$$

Recall that $z^{(k)}$ is cut off by a constraint of $B_{\infty}(0, R)$ with normal $n\left(z^{(k)}\right)$ of length $\left\|n\left(z^{(k)}\right)\right\|=\beta$. Hence $z^{(k) \top} n\left(z^{(k)}\right)$ is the length of the projection of $z^{(k)}$ onto $n\left(z^{(k)}\right)$, which is at least $\beta R$.

So we have

$$
\begin{aligned}
\eta L R & \geq \sum_{k \in J} \lambda_{k} \cdot \gamma^{(k)}(0)+\sum_{k \notin J} \lambda_{k} \cdot \gamma^{(k)}(0) \\
& \geq-\sqrt{n+m} L R \sum_{k \in J} \lambda_{k}+\left(1-\sum_{k \in J} \lambda_{k}\right) \cdot \beta R .
\end{aligned}
$$

Using $\beta \geq 3 \sqrt{n+m} L$, we have $\sum_{k \in J} \lambda_{k} \geq \frac{3 \sqrt{n+m}-\eta}{4 \sqrt{n+m}}>\frac{1}{2}$. Now the result follows from (18).

Thus, given that the maximum of the function $\gamma(z)$ is small, we can find some convex combination of the $z^{(k)}$ 's in the cutting plane method that is a good approximation to the saddle point of $f$. And it turns out that the maximum of $\gamma$ goes to 0 as $T \rightarrow \infty$, and at the same convergence rate as the cutting plane method for convex minimization. 
Lemma C.8 (Upper Bound on $\gamma$ ). Consider solving the convex-concave game by the cutting plane method. Assume that at certain step $T$ we have

$$
\eta=\frac{\operatorname{vol}\left(P^{(T)}\right)^{1 /(n+m)}}{\operatorname{vol}(\mathcal{X} \times \mathcal{Y})^{1 /(n+m)}}<\frac{1}{2} .
$$

Assume that $\beta>L$, we have

$$
\max _{z \in \mathbb{R}^{n+m}} \gamma(z) \leq 8 \eta \beta R(n+m)^{1 / 2}
$$

Proof. Denote $G=\mathcal{X} \times \mathcal{Y}$ for simplicity. First, we note that $\gamma(z)<\beta\left(\|x\|_{\infty}-R\right) \leq 0$ for $z \notin B_{\infty}(0, R)$ since we include all constraints of $\partial B_{\infty}(0, R)$ into the definition of $\gamma$. Hence, it suffices to consider $\gamma$ over $B_{\infty}(0, R)$.

Let $z^{*} \in \mathbb{R}^{n+m}$ be a maximizer of $\gamma$ over $B_{\infty}(0, R)$. Let $\alpha=2 \eta$ and

$$
G^{\alpha}=z^{*}+\alpha\left(G-z^{*}\right):=\left\{(1-\alpha) z^{*}+\alpha z \mid z \in G\right\} .
$$

Notice that $\operatorname{vol}\left(G^{\alpha}\right)=\alpha^{m+n} \operatorname{vol}(G)>\operatorname{vol}\left(P^{(T)}\right)$. Therefore, there is some $w \in G^{\alpha} \backslash P^{(T)}$. This point $w$ is cut off by some $\gamma^{(k)}(z)$ :

$$
\left(w-z^{(k)}\right)^{\top} g^{(k)}>0
$$

for some $z^{(k)} \in B_{\infty}(0, R)$ (by Line 7 of the algorithm). It follows that

$$
\gamma^{(k)}(w)=\left(z^{(k)}-w\right)^{\top} g^{(k)}<0
$$

Since $w=(1-\alpha) z^{*}+\alpha z \in \mathbb{R}^{n+m}$ for some $z \in G$ and the function $\gamma^{(k)}: \mathbb{R}^{n+m} \rightarrow \mathbb{R}$ is affine, we have

$$
(1-\alpha) \cdot \gamma^{(k)}\left(z^{*}\right) \leq-\alpha \cdot \gamma^{(k)}(z)+\gamma^{(k)}(w) \leq-\alpha \cdot \gamma^{(k)}(z)=-\alpha \cdot\left(z^{(k)}-z\right)^{\top} g^{(k)} \leq 2 \alpha \beta R(n+m)^{1 / 2},
$$

where we used $\left\|g^{(k)}\right\| \leq \max (\beta, L) \leq \beta$ and both $z^{(k)}$ and $z$ are in $B_{\infty}(0, R)$. It follows that

$$
\max _{z \in \mathbb{R}^{n+m}} \gamma(z)=\gamma\left(z^{*}\right) \leq 4 \alpha \beta R(n+m)^{1 / 2} \leq 8 \eta \beta R(n+m)^{1 / 2}
$$

Combining Theorem C.4, Lemma C.6, C.7 and C.8, we obtain the following theorem which states that solving convex-concave games is exactly as fast as minimizing convex functions.

Theorem C.9. Given convex sets $\mathcal{X} \subset B(0, R) \subset \mathbb{R}^{n}$ and $\mathcal{Y} \subset B(0, R) \subset \mathbb{R}^{m}$ such that both $\mathcal{X}$ and $\mathcal{Y}$ contain a ball of radius $r$. Let $f(x, y): \mathcal{X} \times \mathcal{Y} \rightarrow \mathbb{R}$ be an L-Lipschitz function that is convex in $x$ and concave in $y$. For any $0<\epsilon \leq \frac{1}{2}$, we can find $(\widehat{x}, \widehat{y})$ such that

$$
\max _{y \in \mathcal{Y}} f(\widehat{x}, y)-\min _{x \in \mathcal{X}} f(x, \widehat{y}) \leq \epsilon L r
$$

in time

$$
O\left((n+m)^{3} \log \left(\frac{n+m}{\epsilon} \frac{R}{r}\right)+(n+m) \log \left(\frac{n+m}{\epsilon} \frac{R}{r}\right) \cdot \mathcal{T}\right)
$$

with high probability in $n+m$ where $\mathcal{T}$ is the cost of computing subgradient $\nabla f$. 
Proof. We run our cutting plane method for $T=(n+m) \log \left(\frac{n+m}{\epsilon} \frac{R}{r}\right)$ iterations. By Theorem C.4, we obtain $P^{(T)}$ with volume

$$
\operatorname{vol}\left(P^{(T)}\right) \leq\left(\frac{\epsilon}{n+m} \frac{r}{R}\right)^{100(m+n)} \cdot \operatorname{vol}\left(P^{(0)}\right) \leq\left(\frac{\epsilon}{n+m} \frac{r}{R}\right)^{99(m+n)} \cdot \operatorname{vol}(\mathcal{X} \times \mathcal{Y})
$$

in $O\left((n+m)^{3} \log \left(\frac{n+m}{\epsilon} \frac{R}{r}\right)\right)$ time. Notice that

$$
\eta=\frac{\operatorname{vol}\left(P^{(T)}\right)^{1 /(n+m)}}{\operatorname{vol}(\mathcal{X} \times \mathcal{Y})^{1 /(n+m)}} \leq\left(\frac{\epsilon}{n+m} \frac{r}{R}\right)^{99}<1 / 2 .
$$

Lemma C.8 shows that

$$
\max _{z \in \mathbb{R}^{n+m}} \gamma(z) \leq 8 \eta \beta R(n+m)^{1 / 2}
$$

Lemma C.6 shows in $O\left((n+m)^{\omega+o(1)} \log \left(\frac{n+m}{\eta}\right)\right)=O\left((n+m)^{\omega+o(1)} \log \left(\frac{n+m}{\epsilon} \frac{R}{r}\right)\right)$ time, we can find $\lambda$ such that

$$
\sum \lambda_{k} \gamma^{(k)}(z) \leq 8 \eta \beta R(n+m)^{1 / 2}+\eta \beta R \leq 9 \eta \beta R(n+m)^{1 / 2}
$$

for all $z \in B_{\infty}(0, R)$. Using this $\lambda$, Lemma C.7 shows that, by taking a convex combination w.r.t. $\lambda$, we can find $(\widehat{x}, \widehat{y})$ for which

$$
\bar{f}(\widehat{x})-\underline{f}(\widehat{y}) \leq 18 \eta \beta R(n+m)^{1 / 2} .
$$

Picking $\beta=3 \sqrt{n+m} L$, we get

$$
\bar{f}(\widehat{x})-\underline{f}(\widehat{y}) \leq 54 \eta L R(n+m) \leq 54 \epsilon L r .
$$

Now our result follows by replacing $\epsilon$ with $\epsilon / 54$, which doesn't change the asymptotic runtime.

Next we give a slightly refined runtime in the case where the domain is a ball using a result of Nemirovski [Nem95].

Corollary C.10. Given an L-Lipschitz function $f(x, y): \mathcal{X} \times \mathcal{Y} \rightarrow \mathbb{R}$ that is convex in $x$ and concave in $y$ with $\mathcal{X}=B(0, R) \subset \mathbb{R}^{n}$ and $\mathcal{Y}=B(0, R) \subset \mathbb{R}^{m}$. For any $0<\epsilon \leq \frac{1}{2}$, we can find $(\widehat{x}, \widehat{y})$ such that

$$
\max _{y \in \mathcal{Y}} f(\widehat{x}, y)-\min _{x \in \mathcal{X}} f(x, \widehat{y}) \leq \epsilon L R
$$

in time

$$
O\left((n+m)^{3} \log (1 / \epsilon)+(n+m) \log (1 / \epsilon) \cdot \mathcal{T}\right)
$$

with high probability in $n+m$ where $\mathcal{T}$ is the cost of computing $\nabla f$.

Proof. By [Nem95, Theorem 5.5.4], we can minimize such a function in time $O\left(\frac{\mathcal{T}+n+m}{\epsilon}\right)$. On the other hand, Theorem C.9 shows we can minimize it in time

$$
O\left((n+m)^{3} \log ((n+m) / \epsilon)+(n+m) \log ((n+m) / \epsilon) \cdot \mathcal{T}\right) .
$$

By using the first result when $\epsilon \geq \frac{1}{n+m}$ and using the second result when $\epsilon \leq \frac{1}{n+m}$, we have the promised runtime. 


\section{Applications of Cutting Plane Method}

\section{D.1 Linear Arrow-Debreu markets}

\begin{tabular}{|l|l|l|l|l|}
\hline Reference & Year & Number of Operations & Time per Operation & Poly Type \\
\hline [Eav75] & 1975 & Finite & Polynomial & Not poly \\
\hline [Jai07] & 2007 & Polynomial & Polynomial & Weakly poly \\
\hline [Ye08] & 2008 & $O\left(n^{6} \log (n U)\right)$ & $M(n \log (n U))$ & Weakly poly \\
\hline [DPSV08] & 2008 & Polynomial & Polynomial & Weakly poly \\
\hline [DM15] & 2015 & $O\left(n^{9} \log (n U)\right)$ & $M(n \log (n U))$ & Weakly poly \\
\hline [DGM16] & 2016 & $O\left(n^{6} \log ^{2}(n U)\right)$ & $M(n \log (n U))$ & Weakly poly \\
\hline [GV19] & 2019 & $O\left(m n^{9} \log ^{2} n\right)$ & $M(n \log (n U))$ & Strongly poly \\
\hline Our result & 2019 & $O\left(m n^{2} \log (n U)\right)$ & $M(n \log (n U))$ & Weakly poly \\
\hline
\end{tabular}

Table 9: (More detailed version of Table 2) Linear Arrow-Debreu Markets. Let $n$ denote the number of agents and $m$ the number of edges. $M(l)$ denotes the time to perform a basic arithmetic operations on $l$-bit numbers.

Linear Exchange (Arrow-Debreu) Markets The input consists of $n$ agents where each agent $i \in[n]$ has a unit of divisible good $g_{i}$ and utility $u_{i, j} \geq 0$ for a unit of good $g_{j}$. The output is a set of prices $p:[n] \rightarrow \mathbb{R}_{+}$for the goods and allocations $x:[n] \times[n] \rightarrow \mathbb{R}_{+}$of the goods to agents. Notice that $x_{i, j}$ can be understood as the amount of good $g_{j}$ perchased by agent $i$, and $x_{i, i}$ is the amount of good $g_{i}$ that agent $i$ keeps to himself.

Market equilibrium By a market equilibrium, we mean a set of prices $p:[n] \rightarrow \mathbb{R}_{+}$and allocations $x:[n] \times[n] \rightarrow \mathbb{R}_{+}$satisfying the following conditions:

- $\sum_{i \in[n]} x_{i, j}=1$ for every agent $j \in[n]$, i.e. every good is fully sold.

- $p_{i}=\sum_{j \in[n]} x_{i, j} p_{j}$ for every agent $i \in[n]$, i.e. the money spent by agent $i$ equals to his income $p_{i}$.

- $p_{i}>0$ for every $i \in[n]$, i.e. prices are positive.

- $\forall i \in[n]$, if $x_{i, j}>0$ then $u_{i, j} / p_{j}=\max _{j^{\prime} \in[n]} u_{i, j^{\prime}} / p_{j^{\prime}}$, i.e. agent $i$ only buys good that attains the best bang-per-bucks.

Assumption D.1. To ensure the existence of an equilibrium, we assume the following. Consider the directed graph $G=(V, E)$ where $V=[n]$ and directed edges $E=\left\{(i, j): u_{i, j}>0\right\}$.

- For each agent $i$, there exist $j, j^{\prime} \in[n]$ such that $u_{i, j}>0$ and $u_{j^{\prime}, i}>0$, i.e. each $i \in G$ has at least one incoming edge and one outgoing edge.

- For every strongly connected component $S \subseteq G$, if $|S|=1$ then there is a loop incident to the node in $S$.

Theorem D.2. A market equilibrium always exists under the above assumptions. 
Previous work The celebrated result of Arrow and Debreu [AD54] shows the existence of a market equilibrium for a broad class of utility functions. From the computational aspects, computing the equilibrium of a market equilibrium in the case of linear utility functions admits a long line of research (see Table 9), leading to both weakly and strongly polynomial runtimes.

A convex formulation The problem of computing a market equilibrium in linear exchange markets can be formulated as the following convex program due to [DGV16], with variables $p_{i}$ representing the prices, $\beta_{i}$ the inverse best bang-per-bucks, and $y_{i j}$ the money paid by agent $i$ to agent $j$. Given $u \in \mathbb{R}_{+}^{n \times n}$ as an input matrix, the goal is to solve the following minimization problem,

$$
\begin{aligned}
\min _{p \in \mathbb{R}^{n}, \beta \in \mathbb{R}^{n}, y \in \mathbb{R}^{n \times n}} & \sum_{i \in[n]} p_{i} \log \left(p_{i} / \beta_{i}\right)-\sum_{(i, j) \in E} y_{i, j} \log u_{i, j} \\
\text { subject to } & \sum_{i:(i, j) \in E} y_{i, j}=p_{j} \quad \forall j \in[n] \\
& \sum_{j: i j \in E} y_{i j}=p_{i} \quad \forall i \in[n] \\
& u_{i j} \beta_{i} \leq p_{j} \quad \forall(i, j) \in E \\
& p_{i} \geq 1 \quad \forall i \in[n] \\
& y, \beta \geq 0
\end{aligned}
$$

Theorem D.3 (Theorem 1 in [DGV16]). Consider an instance of the linear exchange market given by the graph $([n], E)$ and the utilities $u:[n] \times[n] \rightarrow \mathbb{R}_{+}$. The above convex program is feasible if and only if the above assumptions hold, and in this case the optimum value is 0 and the prices $p_{i}$ in an optimal solution give a market equilibrium with allocations $x_{i j}=y_{i j} / p_{j}$.

Convex-concave game formulation The number of variables in the above convex program can be as large as $n^{2}$ and thus a naive application of the cutting plane method would lead to lead to a large runtime. To solve the convex program more efficiently, we transform it into a convex-concave game with a reduced number of variables. Let $\lambda, \eta \in \mathbb{R}^{n}$ be the Lagrange multiplier of constraint 19 and 20 respectively. We have that the above convex program is equivalent to:

$$
\begin{aligned}
\min _{p \geq 1 \in \mathbb{R}^{n}, \beta \in \mathbb{R}_{+}^{n}, u_{i j} \beta_{i} \leq p_{j}} \min _{y \in \mathbb{R}_{+}^{n \times n}} \max _{\lambda, \eta \in \mathbb{R}^{n}} & \sum_{i=1}^{n} p_{i} \log \left(p_{i} / \beta_{i}\right)-\sum_{i=1}^{n} \sum_{j=1}^{n} y_{i, j} \log u_{i, j} \\
& +\sum_{j=1}^{n} \lambda_{j}\left(\sum_{i=1}^{n} y_{i, j}-p_{j}\right)+\sum_{i=1}^{n} \eta_{i}\left(\sum_{j=1}^{n} y_{i, j}-p_{i}\right) .
\end{aligned}
$$

which is equivalent to

$$
\begin{aligned}
\min _{p \geq 1, \beta \geq 0, u_{i j} \beta_{i} \leq p_{j}} \max _{\lambda, \eta \in \mathbb{R}^{n}} \min _{y \geq 0} & \sum_{i=1}^{n} p_{i} \log \left(p_{i} / \beta_{i}\right)+\sum_{i=1}^{n} \sum_{j=1}^{n} y_{i, j}\left(\lambda_{j}+\eta_{i}-\log u_{i, j}\right) \\
& -\sum_{j=1}^{n} \lambda_{j} p_{j}-\sum_{i=1}^{n} \eta_{i} p_{i}
\end{aligned}
$$


which is further equivalent to the following convex-concave game

$$
\min _{p \geq 1, \beta \geq 0, u_{i j} \beta_{i} \leq p_{j}} \max _{\lambda_{j}+\eta_{i} \geq \log u_{i, j}} \sum_{i=1}^{n} p_{i} \log \left(p_{i} / \beta_{i}\right)-\sum_{j=1}^{n} \lambda_{j} p_{j}-\sum_{i=1}^{n} \eta_{i} p_{i}
$$

Notice that the convex-concave game formulation above has $O(n)$ variables. The following upper bound on equilibrium prices is due to [DGV16].

Lemma D.4 (Lemma 13 and Remark 14 in [DGV16]). Assume all utilities are integers $\leq U$ and we let $\Delta=(n U)^{n}$. Then there exists equilibrium prices $p$ that are quotient of two integers $\leq \Delta$, along with allocations $x$ that are quotients of two integers $\leq \Delta^{2}$.

Now using Theorem C.9, we need $O\left(n^{2} \log (n U)\right)$ iterations and the first order oracle requires $O(m)$ operations. Therefore, the total number of operations is $O\left(m n^{2} \log (n U)\right)$. We formally state our result as follows.

Theorem D.5 (Formal version of Theorem 1.4). There exists a weakly polynomial algorithm that computes a market equilibrium in linear exchange markets in time $O\left(m n^{2} \log (n U)\right)$.

\section{D.2 Fisher markets with spending constraint utilities}

\begin{tabular}{|l|l|l|l|l|}
\hline Reference & Year & Running Time & Time per Operation & Poly Type \\
\hline [Vaz10] & 2010 & $O\left(n^{3}(n+m)^{2} \log (U)\right) \cdot \mathcal{T}_{\text {max-flow }}$ & $M(n \log (n U))$ & Weakly poly \\
\hline [Vég16] & 2016 & $O\left(m n^{3}+m^{2}(m+n \log (n)) \log (m)\right)$ & $M(n \log (n U))$ & Strongly poly \\
\hline [Wan16] & 2016 & $O\left(m^{3} n+m^{2} \log (n)(n \log (n)+m)\right)$ & $M(n \log (n U))$ & Strongly poly \\
\hline Our result & 2019 & $O\left(m n^{2} \log (n U)\right)$ & $M(n \log (n U))$ & Weakly poly \\
\hline
\end{tabular}

Table 10: (More detailed version of Table 3) Fisher Markets with Spending Constraint Utilities. Let $n$ denote the total number of buyers and sellers, and $m$ the total number of segments. $\mathcal{T}_{\text {max-flow }}$ denotes the number of operations needed for a max-flow computation. $M(l)$ denotes the time to perform a basic arithmetic operations on $l$-bit numbers.

Fisher markets with spending constraint utilities In the Fisher market model with spending constraint utilities, there are $n_{B}$ buyers and $n_{G}$ perfectly divisible goods. There is a unit ${ }^{4}$ supply of each good. Each buyer $i \in\left[n_{B}\right]$ has a budget $B_{i}$. The utility of a buyer depends on the prices, as follows. The utility is additive across goods, that is, the total utility for a bundle of goods is the sum of utilities for each good separately. For a given good, the utility function is divided into segments; each segment $l$ has a constant rate of utility $u_{i, j, l}$, and a budget $B_{i, j, l}$. The utility of the buyer for $x_{i, j, l}$ amount of good $j \in\left[n_{G}\right]$ under segment $l \in[L]$ is $u_{i, j, l} x_{i, j, l}$, but is subject to the constraint that $p_{j} x_{i, j, l} \leq B_{i, j, l}$. Denote $n:=n_{B}+n_{G}$ the total number of buyers and goods, and $m$ the total number of segments among all pair $(i, j)$.

\footnotetext{
${ }^{4}$ This is without loss of generality since the goods are divisible.
} 
Market equilibrium An allocation $x \in \mathbb{R}_{+}^{n_{B} \times n_{G} \times L}$ and price vector $p \in \mathbb{R}_{+}^{n_{G}}$ are an equilibrium if two conditions are satisfied. The first condition, buyer optimality, is that given $p$, each player maximizes his utility subject to his budget constraint. In other words, for all $i \in\left[n_{B}\right]$, the allocation $x$ optimizes the following program (where $p$ is a constant):

$$
\begin{aligned}
\max _{x \in \mathbb{R}_{+}^{n_{B} \times n_{G} \times L}} & \sum_{j=1}^{n_{G}} \sum_{l=1}^{L} u_{i, j, l} x_{i, j, l} \\
\text { subject to } & \sum_{j=1}^{n_{G}} \sum_{l=1}^{L} p_{j} x_{i, j, l} \leq B_{i}, \\
& 0 \leq x_{i, j, l} \text { and } p_{j} x_{i, j, l} \leq B_{i, j, l} \quad \forall j \in\left[n_{G}\right], l \in[L] .
\end{aligned}
$$

The second equilibrium condition, market clearance, is that $\sum_{i, l} x_{i, j, l}=1$ for all $j \in\left[n_{G}\right]$.

Previous work The Fisher market with spending constraint utilities problem was introduced in [Vaz10] where they gave a weakly poly time algorithm that takes $O\left(n^{3}(n+m)^{2} \log U\right)$ max-flow computations, where $U=\max _{i \in\left[n_{B}\right], j \in\left[n_{G}\right], l \in[L]} u_{i, j, l}$. A convex formulation of the problem was first given in the unpublished manuscript [BDX10]. A strongly poly time algorithm (which is also the best weakly polynomial running time) was given in [Vég16]which achieves $O\left(m n^{3}+m^{2}(m+n \log n) \log m\right)$ number of operations.

A convex formulation The problem of computing a market equilibrium in Fisher markets with spending constraint utilities problem can be captured by the following convex program due to [BDX10].

$$
\begin{array}{rlr}
\max _{b \in \mathbb{R}_{+}^{n_{B} \times n_{G} \times L}, p \in \mathbb{R}_{+}^{n_{G}}} & \sum_{i=1}^{n_{B}} \sum_{j=1}^{n_{G}} \sum_{l=1}^{L} b_{i, j, l} \log u_{i, j, l}-\sum_{j=1}^{n_{G}} p_{j} \log p_{j} & \\
\text { subject to } & \sum_{i=1}^{n_{B}} \sum_{l=1}^{L} b_{i, j, l}=p_{j}, & \forall j \in\left[n_{G}\right], \\
& \sum_{j=1}^{n_{G}} \sum_{l=1}^{L} b_{i, j, l}=B_{i}, & \forall i \in\left[n_{B}\right], \\
& 0 \leq b_{i, j, l} \leq B_{i, j, l}, & \forall i \in\left[n_{B}\right], j \in\left[n_{G}\right], l \in[L] .
\end{array}
$$

Theorem D.6 ([BDX10]). An optimum solution to the above convex program corresponds to an equilibrium for the Fisher market with spending constraint utilities with allocation given by $x_{i, j, l}=$ $b_{i, j, l} / p_{j}$.

Convex-concave game formulation We transform the convex program above to a convexconcave saddle point which allows us to apply the cutting plane method. Let $\eta_{j}, \lambda_{i}$ be the Lagrange multipliers for the two equality constraints and $\mu_{i, j, l} \geq 0$ be the Lagrange multipliers for the inequalities $b_{i, j, l} \leq B_{i, j, l}$ in the above convex formulation. We have the above convex program is 
equivalent to

$$
\begin{aligned}
& \max _{p \geq 0} \max _{b \geq 0} \min _{\mu \geq 0, \eta, \lambda}-\sum_{j=1}^{n_{G}} p_{j} \log p_{j}+\sum_{i, j, l} b_{i, j, l} \log u_{i, j, l} \\
& +\sum_{j=1}^{n_{G}} \eta_{j}\left(p_{j}-\sum_{i, l} b_{i, j, l}\right)+\sum_{i=1}^{n_{B}} \lambda_{i}\left(B_{i}-\sum_{j, l} b_{i, j, l}\right)+\sum_{i, j, l} \mu_{i, j, l}\left(B_{i, j, l}-b_{i, j, l}\right) \\
= & \max _{p \geq 0} \min _{\mu \geq 0, \eta, \lambda} \max _{b \geq 0}-\sum_{j=1}^{n_{G}} p_{j} \log p_{j}+\sum_{i, j, l} b_{i, j, l}\left(\log u_{i, j, l}-\eta_{j}-\lambda_{i}-\mu_{i, j, l}\right) \\
& +\sum_{j=1}^{n_{G}} \eta_{j} p_{j}+\sum_{i=1}^{n_{B}} \lambda_{i} B_{i}+\sum_{i, j, l} \mu_{i, j, l} B_{i, j, l} \\
= & \max _{p \geq 0}{ }_{\mu \geq 0, \eta, \lambda, \eta_{j}+\lambda_{i}+\mu_{i, j, l} \geq \log u_{i, j, l}}-\sum_{j} p_{j} \log p_{j} \\
& +\sum_{j=1}^{n_{G}} \eta_{j} p_{j}+\sum_{i} \lambda_{i} B_{i}+\sum_{i, j, l} \mu_{i, j, l} B_{i, j, l} \\
= & \max _{p \geq 0} \min _{\eta, \lambda}-\sum_{j=1}^{n_{G}} p_{j} \log p_{j} \\
& +\sum_{j=1}^{n_{G}} \eta_{j} p_{j}+\sum_{i=1}^{n_{B}} \lambda_{i} B_{i}+\sum_{i, j, l} B_{i, j, l} \max \left\{0, \log u_{i, j, l}-\eta_{j}-\lambda_{i}\right\},
\end{aligned}
$$

where the last step is because $B_{i, j, l} \geq 0$. Now applying Theorem C.9, we need $O\left(n^{2} \log (n U)\right)$ iterations and the first order oracle takes $O(m)$ operations. So the total number of operations is $O\left(m n^{2} \log (n U)\right)$. We formally state our result as follows:

Theorem D.7 (Formal version of Theorem 1.5). There exists a weakly polynomial algorithm that computes a market equilibrium in Fisher markets with spending constraint utilities in time $O\left(m n^{2} \log (n U)\right)$.

\section{D.3 Walrasian equilibrium for general buyer valuations and fixed supply}

\begin{tabular}{|l|l|l|}
\hline Reference & Year & Running Time \\
\hline$[$ KJC82 $]$ & 1982 & Not polynomial time \\
\hline$[$ Par99 $]$ & 1999 & pseudo-polynomial time \\
\hline [PU02] & 2002 & pseudo-polynomial time \\
\hline$[$ AM02] & 2002 & pseudo-polynomial time \\
\hline d dVSV07] & 2007 & pseudo-polynomial time \\
\hline$[$ LW17] & 2017 & $O\left(n^{2} \mathcal{T}_{\mathrm{AD}} \log (S M n)+n^{6} \log O(1)(S M n)\right)$ \\
\hline Our result & 2019 & $O\left(n^{2} \mathcal{T}_{\mathrm{AD}} \log (S M n)+n^{4} \log (S M n)\right)$ \\
\hline
\end{tabular}

Table 11: (More detailed version of Table 4) Walrasian equilibrium for general buyer valuations and fixed supply.

Recently Paes Leme and Wong [LW17] studied the problem of computing Walrasian equilibrium in a market with arbitrary buyers' valuation functions $v_{i}$ and fixed supply $s$ of indivisible goods. 
They showed that such equilibria are characterized by the minima of the following convex program, which can be solved in polynomial time via a suitable extension of the cutting plane methods.

Let $[m]$ and $[n]$ be the set of buyers and goods respectively. The setting of the problem is as follows.

Buyers' valuation and utility Each buyer $i \in[m]$ may have an arbitrary valuation $v_{i}: \mathbb{N}^{n} \rightarrow \mathbb{R}$ over goods, i.e. for $x \in \mathbb{N}^{n}, v_{i}(x)$ is the valuation of $i$ if she gets $x_{j}$ units of good $j \in[n]$. Given prices $p \in \mathbb{R}^{[n]}$ on goods, the utility of $i$ getting $x \in \mathbb{N}^{n}$ is given by $v_{i}(x)-p \cdot x$. To maximize her utility, given $p, i$ would buy a bundle

$$
x^{(i)} \in \arg \max _{x \in \mathbb{N}^{n}: 0 \leq x \leq s} v_{i}(x)-p \cdot x .
$$

Aggregate demand oracle To study this problem computationally, one must specify the information about the market available to the algorithm. Paes Leme and Wong showed that this problem can be solved in polynomial time assuming the aggregate demand oracle.

Given prices $p$, the aggregate demand is given by $\sum_{i \in[m]} x^{(i)}$, where

$$
x^{(i)} \in \arg \max _{x \in \mathbb{N}^{n}: 0 \leq x \leq s} v_{i}(x)-p \cdot x
$$

is a utility-maximizing bundle for buyer $i$.

Walrasian equilibrium In this economy the supply of goods is fixed at $s \in \mathbb{N}^{n}$. Prices $p$ are said to be at equilibrium if there exist some aggregate demand that matches the supply, i.e.

$$
s=\sum_{i \in[m]} x^{(i)} .
$$

Convex programming One approach to computing Walrasian equilibrium is by formalating the problem as the following convex program. Previous works applied subgradient descent methods to this program to obtain pseudopolynomial time algorithms [Par99, PU02, AM02]. In contrast, Paes Leme and Wong showed that this program can in fact be solved in weakly polynomial time.

$$
\begin{aligned}
& \min _{u, p} \sum_{i \in[m]} u_{i}+p \cdot s \\
& u_{i} \geq v_{i}(x)-p \cdot x \quad \forall i \in[m], \forall x
\end{aligned}
$$

Here $p$ denotes the prices and $u_{i}$ can be thought of as the utility of buyer $i, \forall i \in[m]$. This convex program can clearly be solved with access to the demand oracle of each buyer $i$, i.e. given $p$, return $\arg \max _{x} v_{i}(x)-p \cdot x$, which serves as a separation oracle.

The main result of Paes Leme and Wong is that this can be done via the weaker aggregate demand oracle, i.e. given $p$, return $\sum_{i} x^{(i)}$, where

$$
x^{(i)} \in \arg \max _{x} v_{i}(x)-p \cdot x .
$$

Their algorithm is based on a suitable extension of standard cutting plane methods: 
Theorem D.8 ([LW17]). There is an algorithm that runs in time

$$
O\left(n^{2} \mathcal{T}_{\mathrm{AD}} \log (S M n)+n^{6} \log ^{O(1)}(S M n)\right)
$$

to compute Walrasian prices whenever it exists using only access to an aggregate demand oracle. Here $T_{A D}$ denotes the runtime of the aggregate demand oracle, $n$ denotes the number of goods, $M=\max _{i \in[m], 0 \leq x \leq s}\left|v_{i}(x)\right|$ and $S=\max _{j \in[n]} s_{j}$ ( $s$ and $v_{i}$ are integer-valued).

As in previous applications, $n^{2}$ iterations of cutting plane are required as $\epsilon$ has to be taken to be exponentially small which results in an overall runtime overhead of $\widetilde{O}\left(n^{6}\right)$. By leveraging our faster cutting plane method, we obtain the following improved result:

Theorem D.9 (Formal version of Theorem 1.6). There is an algorithm that runs in time

$$
O\left(n^{2} \mathcal{T}_{\mathrm{AD}} \log (S M n)+n^{4} \log (S M n)\right)
$$

to compute Walrasian prices whenever it exists using only access to an aggregate demand oracle.

Proof. Same as Paes Leme-Wong except that we invoke our cutting plane method for convex minimization in Theorem C.1 instead of LSW's. 